Published in final edited form as:

Small. 2019 June ; 15(23): e1805510. doi:10.1002/smll.201805510.

\title{
3D Bioprinting: from Benches to Translational Applications
}

\author{
Marcel Alexander Heinrich ${ }^{\dagger}$, \\ Division of Engineering in Medicine, Department of Medicine, Brigham and Women's Hospital, \\ Harvard Medical School, Cambridge, MA 02139, USA \\ Department of Biomaterials Science and Technology, Section Targeted Therapeutics, Technical \\ Medical Centre, University of Twente, Enschede 7500AE, The Netherlands \\ Wanjun Liu', \\ Division of Engineering in Medicine, Department of Medicine, Brigham and Women's Hospital, \\ Harvard Medical School, Cambridge, MA 02139, USA \\ Key Laboratory of Textile Science and Technology, College of Textiles, Donghua University, \\ Shanghai 201620, P.R. China

\section{Andrea Jimenez,} \\ Division of Engineering in Medicine, Department of Medicine, Brigham and Women's Hospital, \\ Harvard Medical School, Cambridge, MA 02139, USA \\ Biomedical Engineering Laboratory, Instituto Tecnológico y de Estudios Superiores de Monterrey, \\ Monterrey, Nuevo León 64849, Mexico

\section{Jingzhou Yang,} \\ Division of Engineering in Medicine, Department of Medicine, Brigham and Women's Hospital, \\ Harvard Medical School, Cambridge, MA 02139, USA \\ Center of Biomedical Materials 3D Printing, National Engineering Laboratory for Polymer \\ Complex Structure Additive Manufacturing, Baoding 071000, P.R. China
}

\section{Ali Akpek,}

Division of Engineering in Medicine, Department of Medicine, Brigham and Women's Hospital, Harvard Medical School, Cambridge, MA 02139, USA

Department of Biomedical Engineering, Istanbul Yeni Yuzyil University, Istanbul 34010, Turkey

Xiao Liu,

Division of Engineering in Medicine, Department of Medicine, Brigham and Women's Hospital, Harvard Medical School, Cambridge, MA 02139, USA

Key Laboratory for Biomechanics and Mechanobiology of the Ministry of Education, School of Biological Science and Medical Engineering, Beihang University, Beijing 100191, P.R. China

\section{Qingmeng Pi,}

Division of Engineering in Medicine, Department of Medicine, Brigham and Women's Hospital, Harvard Medical School, Cambridge, MA 02139, USA

\footnotetext{
*Author to whom correspondence should be addressed. yszhang@ research.bwh.harvard.edu. 
Department of Plastic and Reconstructive Surgery, Renji Hospital, Shanghai Jiao Tong University School of Medicine, Shanghai 200129, P.R. China

Xuan Mu,

Division of Engineering in Medicine, Department of Medicine, Brigham and Women's Hospital, Harvard Medical School, Cambridge, MA 02139, USA

Ning Hu,

Division of Engineering in Medicine, Department of Medicine, Brigham and Women's Hospital, Harvard Medical School, Cambridge, MA 02139, USA

Biosensor National Special Laboratory, Key Laboratory of Biomedical Engineering of Education Ministry, Department of Biomedical Engineering, Zhejiang University, Hangzhou 310027, P.R. China

Raymond Michel Schiffelers, Department of Clinical Chemistry and Hematology, University Medical Center Utrecht, Utrecht $3584 \mathrm{CX}$, The Netherlands

Jai Prakash, Department of Biomaterials Science and Technology, Section Targeted Therapeutics, Technical Medical Centre, University of Twente, Enschede 7500AE, The Netherlands

Jingwei Xie, Holland Regenerative Medicine Program, University of Nebraska Medical Center, Omaha, NE 68198, USA

\section{Yu Shrike Zhang}

Division of Engineering in Medicine, Department of Medicine, Brigham and Women's Hospital, Harvard Medical School, Cambridge, MA 02139, USA

\section{Abstract}

Over the last decades, the fabrication of three-dimensional (3D) tissues has become commonplace in tissue engineering and regenerative medicine. However, conventional 3D fabrication techniques such as scaffolding, microengineering, and fiber and cell sheet engineering are limited in their capacity to fabricate complex tissue constructs with the required precision and controllability that is needed to replicate biologically relevant tissues. To this end, 3D bioprinting offers great versatility to fabricate biomimetic volumetric tissues that are structurally and functionally relevant. It enables precise control of the composition, spatial distribution, and architecture of bioprinted constructs facilitating the recapitulation of the delicate shape and structure of targeted organs and tissues. Here we systematically review the history of bioprinting and the most recent advances in instrumentation and methods. We then focus on the requirements for bioinks and cells to achieve optimal fabrication of biomimetic constructs. We next discuss emerging evolutions and future directions of bioprinting such as freeform, high-resolution, multi-material, and four-dimensional bioprinting. Finally, we present the translational potential of bioprinting and bioprinted tissues of various categories and conclude by exemplifying commercially available bioprinting platforms.

\section{Graphical Abstract}


Recent advances in translating 3D bioprinting to the clinics are reviewed, including developments in bioprinting strategies, innovations in bioinks for bioprinting, advances in bioprinting of complex architectures, and translational potential of bioprinted tissue-like structures. Commercially available bioprinting platforms are briefly discussed towards the end.

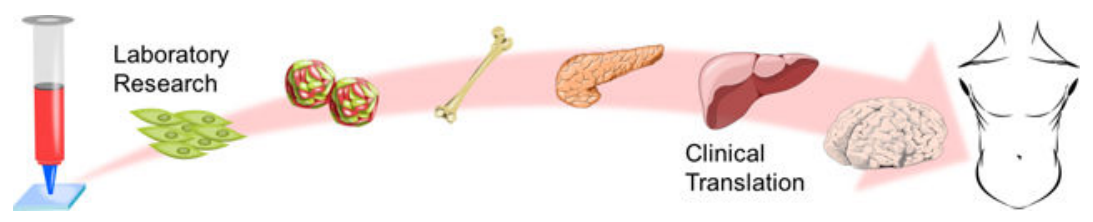

\section{Keywords}

3D bioprinting; additive manufacturing; bioink; tissue engineering; regenerative medicine

\section{Introduction}

Tissue engineering aims to generate and utilize biomimetic tissue or organ substitutes to replace, repair, or augment those damaged by injuries or diseases. ${ }^{[1]}$ The native tissues are typically sophisticated in terms of cellular population, extracellular matrix (ECM) composition, variety of growth factors and bioactive agents, as well as spatial organization of all these different components in an orchestrated manner. ${ }^{[2-4]}$ No tissue is composed of only a single cell type. For example, the skin contains layered keratinocytes and fibroblasts, which have a protective function, while the function of melanocytes is to give color; ${ }^{[5,6]}$ the heart beats upon contraction of cardiomyocytes initiated by pacemaker cells lying in the Purkinje fibers; ${ }^{[6-8]}$ the liver functions based primarily on hepatocytes but other nonparenchymal cells, such as biliary epithelial cells, hepatic stellate cells, and Kupffer cells are also indispensable; $[6,9,10]$ and the brain contains billions of neurons surrounded by astrocytes to provide nutrients and glial cells to modulate the immunity. ${ }^{[6]}$ These various cell types are tightly connected by a plethora of ECM molecules in specific spatial arrangements. This allows the cells to interact in the right context with strong coordination mediated by the presence of residing or diffusive growth factors, hormones, and additional bioactive molecules. ${ }^{[2,3,11]}$ Moreover, blood vessels are another critical component of almost all functional tissues. These perfusable networks function to transport nutrients, oxygen, and bioactive agents across different organs or sections of a tissue, and remove metabolic wastes such as acids and carbon dioxide to maintain the homeostasis of the human body. $[12,13]$ Without an interconnected vascular network, tissues cannot survive on their own.

To date, many strategies have been developed to engineer functional tissues, such as threedimensional (3D) scaffolding, ${ }^{[2,14]}$ microengineering based on self-assembly, ${ }^{[15,16]}$ fiber engineering, ${ }^{[17]}$ scaffold-free cell sheet engineering, ${ }^{[18]}$ and others. ${ }^{[3,4]}$ Scaffolds, made from materials including hydrogels and biodegradable polymers, can be processed into $3 \mathrm{D}$ volumes of desired structures, architectures, and shapes to allow seeded cells to attach, proliferate, migrate, and differentiate. ${ }^{[2,19]}$ Microscale building units, including blocks with complementary shapes or surface chemistry (e.g., DNA sequences and hydrophilicity, respectively), can self-assemble into bulk volumes resembling the properties of the target 
tissues. ${ }^{[16,20]}$ Cell-laden fibers may also be used as building units and assembled into hierarchical structures through weaving, knitting, braiding, and spooling. ${ }^{[17,21]}$ Scaffoldfree cell sheet engineering relies on stacking of thin sheets of cells to assume the desired 3D tissue constructs. ${ }^{[18]}$ Although these strategies all possess their own advantages, none of them have been able to achieve reproducible fabrication of volumetric tissue constructs at high spatial precision and controllability.

Three-dimensional bioprinting is a recently developed biofabrication technology capable of addressing such a challenge by providing unprecedented accuracy and precision in patterning biomaterials and cells in a 3D volume in a highly reproducible manner empowered by a programmed robotic fabrication mechanisms. ${ }^{[9,22-24]}$ To date, a variety of bioprinting strategies have been proposed and executed, including those based on stereolithography, extrusion, and droplets, for engineering different types of tissue substitutes and models of interest. This review systematically discusses the history of bioprinting and its recent advancements in both instrumentation and methods. Subsequently, it provides a detailed discussion on the requirements for a selection of bioinks to achieve optimal bioprinting of biomimetic constructs. We next summarize the trends and directions in future development of bioprinting including freeform, high-resolution, multi-material, and four-dimensional (4D) bioprinting. Finally, we conclude with the translational potential of bioprinting technologies and illustrate exiting commercial bioprinting platforms that are possibly capable of meeting such a need.

\section{Bioprinting Strategies}

Additive manufacturing techniques in conjunction with biomaterials and cells enable rapid fabrication of 3D biomimetic structures for applications in tissue engineering and regenerative medicine. Over the past decade, a variety of strategies for 3D bioprinting have been developed (Figure 1), each with their own unique advantages and limitations (Table 1). In this section, we will discuss these 3D bioprinting strategies, their working principles, as well as advantages/disadvantages and suitability for different applications.

\subsection{Stereolithography}

The initial demonstration of stereolithography for additive manufacturing could be dated back to 1986 when Charles W. Hull initially described the process of creating 3D objects by selectively transform the physical state of a fluid to a solid in a reservoir, through layer-bylayer photocrosslinking. ${ }^{[25]}$ In medicine, stereolithography was first used to create models for reconstructive head surgery, where researchers were able to produce highly accurate and detailed models of the cranium. ${ }^{[26]}$ Stereolithography bioprinters are based on the concept to selectively solidify a (cell-laden) bioink using photo-polymerization in a layer-by-layer process controlled by a moveable stage along the z-axis (Figure 1A). In stereolithography, a two-dimensional (2D) pattern of interest is projected onto the bioink reservoir, allowing for the generation of complex 3D structures without the need of a printhead that travels in $x-y$ direction. This feature results in a higher bioprinting speed in comparison with other nozzlebased bioprinters. The selective crosslinking of bioink by light results in no shear stress to cells, enabling these bioprinters to achieve a high cell viability $(>85 \%) .{ }^{[27]}$ One major 
disadvantage of this system is the necessity of the liquid to be transparent with limited scattering; otherwise light would not be able to uniformly pass the material resulting in a non-uniform crosslinking. Due to this requirement, cell density within the bioink is limited to approximately $10^{8}$ cells $\mathrm{mL}^{-1} \cdot{ }^{[27]}$ Despite this disadvantage, stereolithography has drawn the interest of several research fields as a result of its capacity to bioprint structures rapidly without applying shear forces to cells and with high resolution $(\sim 1 \mu \mathrm{m}) .{ }^{[28,29]}$ The detailed advantages of stereolithography as well as recent studies to print biomimetic tissues will be further discussed in Section 5.1.2.

\subsection{Inkjet bioprinting}

Inkjet bioprinting was initially developed in 2003 and officially patented in $2006 .{ }^{[30]}$ It is based on conventional 2D inkjet printers, where the ink is replaced with a bioink and the paper switched to an $\mathrm{x}-\mathrm{y}-\mathrm{z}$ controllable platform allowing for 3D manufacturing of objects. The bioink deposition is based on temporal deformation of the internal space within the extruder, through which the bioink flows, due to piezoelectric actuation or digitized thermal actuation (Figure 1B). This deformation allows for droplet-wise dispensing of liquid onto the collection platform, and the build-up of layers in the z-direction eventually leads to generation of a 3D object. The major advantage of inkjet bioprinting lies in the simplicity of the system and relatively low costs. ${ }^{[27,31]}$ Several printheads can further work in parallel allowing for rapid fabrication at high resolution $(\sim 30 \mu \mathrm{m}) .{ }^{[27,30,32]}$ In addition, this technique results in a relatively high cell viability of $80-90 \% .{ }^{[27,31]}$ However, inkjet bioprinting also displays several disadvantages such as a relatively low cell density $\left(<10^{6}\right.$ cells $\mathrm{mL}^{-1}$ ) that can be used for bioprinting as well as the capacity to only print bioinks with viscosities in a range of around 3.5-12 $\mathrm{mPa} \cdot$ s. $^{[27,30,31,33]}$ The relatively high resolution of the system of approximately $30 \mu \mathrm{m}$, as well as the capacity to use multiple printheads make inkjet bioprinting attractive for multiple applications. Cui and co-workers described an inkjet bioprinter, which was able to deposit 8 materials and simultaneously crosslink the bioprinted structures for studies on bone and cartilage. ${ }^{[32]}$ They successfully developed a platform to achieve mechanically strong bone and cartilage constructs using poly(ethylene glycol) (PEG)-dimethacrylate (PEGDMA) and gelatin methacryloyl (GelMA), and encapsulated human mesenchymal stem cells (MSCs). Huang and co-workers successfully bioprinted bifurcated vascular structures using inkjet bioprinting with a controlled and uniform diameter of the channels. ${ }^{[34]}$ Recently, Jung and co-workers developed a bioprinting process based on a combination of inkjet bioprinting with a spray-coating technique. ${ }^{[35]}$ They demonstrated the versatility of their technique by the rapid fabrication of hydrogel constructs with various sizes and materials including alginate, cellulose, fibrinogen, or GelMA. Bioprinted human dermal fibroblasts in the constructs displayed high proliferation and activity as well as cell-specific characteristics such as extensive collagen I deposition. Their novel approach showed that inkjet bioprinting can be used to bioprint larger-scale constructs consisting of various materials for applications in tissue engineering.

\subsection{Laser-assisted bioprinting}

Laser-assisted bioprinting is based on laser direct-write or laser-induced forward transfer. ${ }^{[36]}$ The first appearance of laser-assisted bioprinting applied to tissue engineering was on a publication by Duocastella and co-workers in 2007, describing the bioprinting of a 
microarray of droplets, laying the foundation for the use of this method in biomedical applications. ${ }^{[37]}$ Platforms using this technique typically consist of three layers from top to bottom: an energy-absorbing layer, a donor layer, and a layer of bioink (Figure 1C). The crucial part of the system is a donor layer that responds to an applied laser beam. Attached on top of this donor layer is an energy-absorbing layer (e.g., titanium or gold). At the bottom, a thin layer of bioink is suspended for bioprinting. When a selective laser beam is applied to desired sites of the energy-absorbing layer, the corresponding locations of the donor layer underneath is vaporized, creating a high-pressure bubble at this interface. This pressure causes impelling of the bioink, resulting in a droplet falling onto the collection platform. By controlling the z-stage of the collector, a 3D construct is eventually formed. Laser-assisted bioprinting has the advantage that cells are not directly exposed to high shear stress. In fact, during the bioprinting process there is no contact between the dispenser and the bioink. As a result, this bioprinting method leads to a high cell viability (>95\%) and is able to also deposit highly viscous materials $(1-300 \mathrm{mPa} \cdot \mathrm{s}) .{ }^{[33]}$ One of the major problem with this system is the high cost due to the requirement of a high-resolution and intensity laser diode. With the drop-wise bioprinting mechanism, the cell density achievable by this method is similar to that of inkjet bioprinting $\left(<10^{6}\right.$ cells $\left.\mathrm{mL}^{-1}\right)$, which has limited its applications to a certain extent. ${ }^{[27]}$ Guillemot and co-workers successfully bioprinted MSCs with a high viability and at a high resolution using a high-throughput laser-assisted bioprinting. ${ }^{[38]}$ Additionally, they developed a system that could achieve cell-level resolution $(10 \mu \mathrm{m})$ at a very high speed $(5 \mathrm{kHz})$ of bioprinting. ${ }^{\left[{ }^{[9]}\right.}$ In summary, laser-assisted bioprinting shows a good potential based on contactless deposition at fast speeds. However, because due to high costs and the fact that not all parameters are completely optimized and understood, laser-assisted bioprinting is still relatively immature for the fabrication of 3D tissue constructs.

\subsection{Extrusion-based bioprinting}

The basic mechanism of extrusion-based 3D printing for tissue engineering applications was first applied by Hutmacher and co-workers back in 2002. ${ }^{[40]}$ They created scaffolds made from poly( $\varepsilon$-caprolactone) (PCL) using an extrusion-based design with two rollers combined with a heating system to deliver melted material. Over the last years, the process of extrusion-based 3D printing rapidly developed and its application soon extended to 3D bioprinting. Several different systems have been developed for extrusion-based bioprinting (Figure 1D); among them, two main working principles have displayed high performance in tissue engineering applications: pneumatic- and mechanical-driven fluid dispensing systems. Pneumatic systems can be either valve-free or valve-based. ${ }^{[41]}$ Valve-free systems, on the one hand, are easier to manufacture and are therefore most commonly used in bioprinting. Whereas valve-based systems, on the other hand, have several advantages in terms of control over pressure and pulse frequencies, enabling high precision of material deposition and resulting in high-resolution bioprinting. Mechanical systems are mainly controlled by a piston or by a screw. Piston-based systems allow for direct control over the bioink deposition onto the platform. Screw-driven systems are preferable for bioinks with higher viscosities due to the better spatial control. However, due to larger pressure drops at the nozzle exit, screw-driven systems can be harmful for cells. ${ }^{[41,42]}$ In comparison with other aforementioned platforms, extrusion-based bioprinting possesses several advantages, 
including the capacity of depositing high-viscosity bioinks (30 $\mathrm{mPa} \cdot \mathrm{s}$ to $>6 \times 10^{7} \mathrm{mPa} \cdot \mathrm{s}$ ) as well as large cell densities (>10 ${ }^{8}$ cells $\mathrm{mL}^{-1}$ up to cell spheroids). ${ }^{[27,33]}$ Moreover, extrusion-based bioprinting systems can continuously extrude bioinks without interruptions. In most applications, this is preferable in comparison to dropwise methods considering the requirement for the integrity of bioprinted tissue constructs. On the other hand, the bioprinting speed is relatively slow to build up a 3D structure and the resolution is strongly dependent on different components such as the nozzle size. Studies have also shown that cell viability after extrusion-based bioprinting is usually moderate at around $40-80 \%$ due to high shear stresses experienced by cells during the extrusion process. ${ }^{[27]}$ Nevertheless, extrusionbased bioprinting has thus far been one of the most widely adopted forms of bioprinting due to its relative simplicity in instrumentation.

\subsection{Electrospinning-based bioprinting (EBB)}

Electrospinning is a simple and versatile method to produce nano- and microscale fibers using various materials including polymers, ceramics, and composites. ${ }^{[43,44]}$ This method uses an electrical force to rapidly stretch a charged polymer solution/melt jet coupled with solvent evaporation/solidification and subsequent collection of filaments into a fibrous mat. ${ }^{[45]}$ Due to the small diameter of electrospun fibers achievable by this technique, electrospinning has been recently modified and employed as an emerging method to improve the resolution of current bioprinting platforms. The setup usually consists of an $x-y-z$ robotic stage combined with a conventional electrospinning equipment set, including a solution or melt polymer extrusion system based on a syringe pump or pneumatic regulator and a highvoltage power supply (Figure 1E). Electrospinning allows the bioprinting to be controlled in either a continuous or discontinuous manner. ${ }^{[46-49]}$

One major disadvantage in electrospinning is the chaotic whipping of the charged jet resulting in unstable fibers and constructs. ${ }^{[46]}$ Up to now, the main strategy to deposit electrospun ultrafine fibers in a controllable manner is utilizing a stable liquid jet region, namely near-field electrospinning (NFES), electrospinning writing, and direct writing, all of which will be further referred to in this section as EBB..$^{[46,50,51]}$ One of the main characteristics of EBB is the shorter collecting distance (usually between $500 \mu \mathrm{m}$ and 3 $\mathrm{mm}$ ), while the collecting distance of conventional electrospinning ranges from a few centimeters to $30 \mathrm{~cm} .{ }^{[44,52]}$ The applied voltage is also lower when compared with conventional electrospinning, but it significantly varies depending on the type of bioprinting.

In general, EBB can be classified as solution electrospinning-based bioprinting (SEBB) and melt electrospinning-based bioprinting (MEBB). The applied voltage of SEBB is a few hundred volts, while the counterpart of MEBB is approximately $10 \mathrm{kV}$. SEBB, on the one hand, can bioprint fibers in the range of nanometers with a smallest diameter reported of $16.2 \mathrm{~nm}$; however, this technique has not yet been proven in biological applications. ${ }^{[47]}$ MEBB, on the other hand, can deposit fibers in the range of $650 \mathrm{~nm}$ to $980 \mathrm{~nm}$ in a highly controlled manner, making it suitable for the fabrication of high-resolution scaffolds for cell attachment and long term culture. ${ }^{[50]}$ Detailed discussions can be found in Section 5.2. 


\section{Design of Functional Bioinks for Bioprinting}

Bioinks are one of the most important aspects for successful 3D bioprinting of engineered tissue and organ constructs. They form the backbone of almost every 3D-bioprinted construct. ${ }^{[53,54-57]}$ In general, bioinks should possess excellent mechanical and biological properties, defining printability and biocompatibility of the bioink. Printability indicates the suitability of a bioink to fabricate stable 3D constructs with high structural integrity and fidelity whereas biocompatibility indicates that the bioink is cell-friendly and supports cell adhesion, proliferation, and spreading. ${ }^{[29]}$ Hereby cells can be seeded on or encapsulated into bioprinted constructs to promote specific tissue growth. Especially in the fabrication of larger-scale biomimetic tissues, the choice of the proper bioink is crucial for the success of bioprinting. The bioink needs to maintain the structure of a bioprinted construct of a larger size, promote cell growth, spreading, and interaction throughout the whole construct, as well as support maturation into respective tissues.

Over the last decades, several naturally derived and synthetic biopolymer-based hydrogels have proven to be promising candidates to serve as bioinks for bioprinting applications. Bioinks that have been extensively investigated include alginate, fibrinogen, gelatin, collagen, chitosan, agarose, pluronics, hyaluronic acid (HA), GelMA, PEG, and decellularized ECM (dECM) ${ }^{[57]}$ These bioinks are ion-sensitive, photosensitive, thermosensitive, enzyme-sensitive, or $\mathrm{pH}$-responsive so that they can be easily gelled to form solid 3D constructs before, during, and/or after bioprinting.

Among all, alginate is perhaps one of the most frequently used bioinks in bioprinting for medical applications and tissue engineering. Its viscous properties, combined with the comparatively simple crosslinking mechanism using calcium chloride $\left(\mathrm{CaCl}_{2}\right)$, make it an attractive hydrogel for bioprinting applications. ${ }^{[56,58]}$ The main disadvantage of alginate is its chemical structure that usually does not allow cell adhesion. To induce cell adhesion and biological activity, alginate needs to be blended with other natural polymers such as gelatinbased materials, collagen, or fibrinogen. ${ }^{[59,60]}$ Gelatin and GelMA are other hydrogels that are widely used in bioprinting applications due to their excellent cell compatibility and mechanical properties. ${ }^{[61]}$ A disadvantage of using GelMA is the requirement of a photoinitiator that allows for crosslinking and solidification of bioprinted constructs. At higher concentrations these initiators can be toxic to cells, which limits the use to low concentrations of photoinitiator. ${ }^{[59]}$

Similar to gelatin, HA can be modified to create a printable bioink usable for cell-laden constructs. These supramolecular HA-based hydrogels are especially attractive due to their tunable characteristics and mechanical properties. ${ }^{[62]}$ Hydrogels based on AECM are attracting increasing interest for the use as bioinks due to their naturally given biocompatibility, tissue specificity, and ease to formulate into usable bioinks. ${ }^{[63]}$ Different from hydrogels that can be directly used as cell-laden bioinks are hydrogels that mainly act as supporting or sacrificial layers, such as pluronics or gelatin. ${ }^{[64]}$ In general, over the last decades, the research on bioinks for bioprinting has been rapidly growing and new combinations and modified bioinks are developed constantly to achieve improved mechanical and biological properties for application in tissue engineering and regenerative 
medicine. This chapter briefly reviews the recent progress of functional bioinks and their applications in 3D bioprinting of specific tissues/organs, which are of particular interest in the fabrication of clinically relevant biomimetic tissue constructs. More systematic discussions on bioinks can be accessed elsewhere. ${ }^{[29,55,57,65]}$

\subsection{Mechanical properties of bioinks}

The fabrication of complex 3D functional structures in bioprinting requires accurate layerby-layer placement and/or solidification of the bioink. During this process, the bioprinted layers need to sustain their shape and hold their previously defined 3D structure. Furthermore, cells loaded in the bioinks should not be negatively influenced by the bioprinting process in terms of viability and performance. ${ }^{[23,66,67]}$ To achieve this goal, it is crucial to take into consideration the rheological properties of the bioinks, the mechanical effects on encapsulated cells, and the function of bioprinted tissues.

As discussed in the previous section, the most commonly used method in bioprinting is extrusion-based deposition of a bioink through a printhead onto a collector in conjunction with a rapid phase transition of the bioink into a solid-like material. ${ }^{[67]}$ Here, the bioink should fulfill different requirements such as a viscosity that is low enough to allow extrusion through the printhead while avoiding high shear rates on laden cells, but high enough to hold a 3D shape after extrusion. Because of these requirements, hydrogels with shear-thinning properties came into focus of many bioprinting applications. Shear-thinning hydrogels display low viscosities when high shear stress is applied to the materials and regain their original viscosities after the shear stress is removed. ${ }^{\left[22,{ }^{68]}\right.}$ Based on their optimal shearthinning properties, bioinks based on alginate or GelMA have been widely applied. ${ }^{[69]}$

Moreover, several research has focused on the hybridization of these bioinks with nanomaterials to achieve improved performances. For example, Gaharwar and co-workers indicated that nanoclay particles in a GelMA-based bioink could increase its shear-thinning properties (Figure 2A). ${ }^{[70]}$ In their study, it was shown that the mechanical properties of GelMA could be fine-tuned by addition of nanoparticles of different sizes and concentrations. Nanoclay addition resulted in a 4-fold increase in the compressive modulus and a 10-fold increase in the peak tensile stress at $90 \%$ strain. The energy absorbed during deformation (90\% cyclic compression) of the GelMA/nanoclay hydrogel was 6-fold higher than that of pure GelMA. Such a bioink can be used to bioprint hydrogel constructs that are highly elastic under high compressive strains. They demonstrated that nanoclay could increase the mechanical properties of bioprinted constructs from biopolymer solution-based bioinks and did not decrease their biocompatibility.

Another strategy to fine-tune the properties of bioinks is a change of bioprinting conditions such as maintaining a relatively high temperature during extrusion that could decrease viscosities of certain bioinks and contribute to easier extrusion of the materials. ${ }^{[71,72]}$ For instance, Dubruel and co-workers demonstrated that the viscosity of GelMA would sharply decrease from 10 Pa.s to $0.01 \mathrm{~Pa}$.s when the bioprinting temperature increased from $20{ }^{\circ} \mathrm{C}$ to $37^{\circ} \mathrm{C} \cdot{ }^{[71]}$ Furthermore, the concentration of GelMA and the density of loaded cells have additional impacts on the viscosity of the bioink. They indicated that an elevated concentration of GelMA caused an increase in the viscosity whereas a higher cell density 
lowered the viscosity of the bioink. Fu and co-workers systemically investigated the influence of the processing parameters such as air pressure, feed rate, and bioprinting distance on the printability of biomaterials. ${ }^{[72]}$ It was found that a higher pressure resulted in a higher feeding rate and a larger volume of bioink being deposited at one location, consequently increasing the line width of the bioprinted structure. The same effect could be seen by slowing the moving speed of the printhead. Conversely, a slower feeding rate and/or higher bioprinting speed could decrease the line width. A different approach to optimize the bioprinting conditions is to reduce the time between material extrusion and phase transition through in situ crosslinking of the bioinks, which decreases the possibility of the structure to collapse. For instance, Khademhosseini and co-workers leveraged a coaxial extrusion printhead to bioprint a low-viscosity bioink consisting of alginate and GelMA. ${ }^{[69]}$ With this extrusion system, they were able to extrude a continuous filament of alginate/GelMA that was surrounded by a solution of $\mathrm{CaCl}_{2}$. At the tip of the coaxial printhead, the bioink underwent rapid phase transition due to the fact that alginate would undergo an instantaneous physical gelation when exposed to calcium ions. Using this method, they bioprinted microfibrous 3D structures at good resolution with a single fiber diameter of down to $100 \mu \mathrm{m}$.

The mechanical properties of the bioink to achieve optimal printability and capability to form a 3D construct is not the only requirement necessary to be suitable for bioprinting applications. An inevitable consideration is also the mechanical effects on loaded cells during the extrusion process. It is well-documented that the mechanical stress has a profound influence on cell survival and function. ${ }^{[73]}$ However, in bioprinting little is known regarding the mechanical effects on laden cells and most reports are still controversial. Khalil and Sun indicated that, when the shear stress applied to cells changed from $100 \mathrm{kPa}$ to $1150 \mathrm{kPa}$, the viability percentage of rat heart endothelial cells could range from $76 \%$ to $83 \%$ and there was no statistically significant difference. ${ }^{[74]}$ In contrast, Heilshorn and coworkers further investigated the mechanical influence on cell viability during the extrusion process. ${ }^{[75]}$ They found that the mechanical force applied on cells during extrusion through the nozzle had an adverse effect on cell viability. In particular, the extensional flow induced by the sharp change in cross-sectional diameter from syringe to nozzle, rather than the pressure drop and shear stress, was the main cause of acute cell death. Recently, Fischer and co-workers precisely controlled the shear stress at the nozzle site during the bioprinting process and investigated the effects of different levels of shear stress on the viability and proliferation of mesenchymal stem cells (MSCs). ${ }^{[76]}$ They showed that loaded cells could be bioprinted without side effects under a threshold of shear stress. However, high shear stresses would affect cell viability during the bioprinting process and furthermore induce long-term alterations in the proliferation of the surviving cells. For this reason, hydrogels with shear-thinning properties are often used, as the low shear stress during extrusion favors cell survival and functionality. ${ }^{[7]}$

However, the mechanical properties of bioinks do not solely affect laden cells in a negative way. These properties may also have a profound positive influence on cell maturation and development and form a crucial factor in obtaining biomimetic tissues for regenerative medicine. For some tissues such as articular cartilages and muscles, the mechanical properties of bioprinted constructs are of utmost importance. Nevertheless, bioinks based on 
a single hydrogel or a blend of two comparably soft hydrogels such as alginate and GelMA are often too weak to be used for bioprinting of musculoskeletal tissues. ${ }^{[68,78]}$

To overcome the limitations of commonly used bioinks, two strategies have been developed recently. The first one is the hybrid bioprinting strategy, in which a reinforcing component is combined with a bioink providing the necessary mechanical support. ${ }^{[79,80]}$ For instance, Malda, Hutmacher, and co-workers 3D-printed high-porosity melt-electrospun PCL fiber scaffolds through EBB to mechanically reinforce GelMA hydrogels resulting in bioprinted constructs that, in terms of mechanical properties, closely resembled those of healthy articular cartilage. ${ }^{[80]}$ Moreover, human chondrocytes embedded in the composites were viable and maintained their cell-specific functions. The second strategy is based on composite bioinks consisting of an intertwined hybrid network providing the necessary mechanical properties. ${ }^{[70,81,82]}$ Zhao and co-workers reported a bioink of alginate-PEGnanoclay for 3D bioprinting of highly stretchable and tough hydrogel structures (Figure 2B). ${ }^{[81]}$ Alginate and PEG polymers were ionically and covalently crosslinked by calcium ions and UV exposure, respectively. As the hydrogels are deformed, alginate chains are detached from the ionic crosslinks so that mechanical energy is dissipated. Once the hydrogels are relaxed from deformation, they regain their original configurations since the covalently crosslinked PEG network retains the elasticity of the hydrogels. Over time, the ionic crosslinks of the alginate network can reform within both deformed and relaxed hydrogels. More complex tough 3D hydrogel structures could be bioprinted through controlling the viscosity of the solution with nanoclay addition. The bioprinted constructs could bear high stress in both tension and compression. A bioprinted grid was uniaxially stretched to $300 \%$ of its length and relaxed to its initial state (Figure 2C). A bioprinted pyramid underwent 99\% compressive strain and recovered $97 \%$ of its original height after unloading in 5 mins (Figure 2D). The constructs achieved a high toughness of above $1,500 \mathrm{~J} \mathrm{~m}^{-2}$. The high viability of human embryonic kidney (HEK) cells seeded on the hydrogel constructs demonstrated that the alginate-PEG-nanoclay bioink was not only printable to form tough structures but also suitable for long-term cell cultures.

Alternatively, Alblas and co-workers described the 3D bioprinting of cell-laden constructs for bone tissue engineering using a modified bioink consisting of methacrylated HA (MeHA). ${ }^{[83]}$ They were able to induce osteogenic differentiation of incorporated MSCs solely based on the mechanical properties of the bioink without the need for additional osteogenic stimuli. Incorporated cells displayed a viability of $\sim 64.4 \%$ after culturing for 21 days, proving the biocompatibility of the bioink. Osteogenic differentiation was measured by quantification of calcium deposition by MSCs. They were able to show a concentrationdependent response based on the used MeHA polymer concentration (Figure 2E). Wei and co-workers more recently developed an alginate-polyvinyl alcohol (PVA)-hydroxyapatite bioink for 3D bioprinting of a scaffold that was relevant for bone tissue engineering. ${ }^{\text {[84] }}$ Their bioink presented optimal rheological and biological properties for bioprinting of bonelike scaffolds encapsulating MC3T3-E1 preosteoblasts. The bioprinted constructs were stable for a duration of 14 days and encapsulated cells displayed high viability as well as osteogenic differentiation throughout the entire constructs. 
In summary, the mechanical properties of the used bioink are crucial for the formation of 3D constructs. Not only do the mechanical properties have influence on the bioprinting properties and the rigidity of the final construct, but they also directly influence cell behavior and activity, including viability and differentiation. However, it remains to be understood in how far the mechanical properties of, in particular, bioinks with high toughness might impede cell proliferation and other crucial aspects of cell behaviors. Bioprinted constructs with high toughness, as aforementioned, are often based on dense polymer networks or rely on additives, which could have a direct effect on cell responses, such as proliferation, or on cell phenotypes. To fully prove the applicability of such hydrogels in the fabrication of biologically relevant tissues, it is crucial to investigate these characteristics in the future.

\subsection{Biological properties of bioinks}

Besides the passive influence of the bioink on laden cells based on mechanical forces, the bioink can also have direct influences on cell viability and behavior. Therefore, the biological properties and the biocompatibility of the bioink are crucial to ensure the generation of target tissues. Numerous bioinks have been used in bioprinting applications that can be loaded with cells to achieve cell-laden bioprinted constructs. The different advantages and disadvantages of specific bioinks in terms of their biological properties were reviewed extensively elsewhere. ${ }^{[29,54,57,65]}$ This section focuses solely on one specific source of highly biocompatible bioinks, which has been of particular interest for its use in bioprinting, i.e., dECM bioinks. In the body, ECM is excreted by resident cells to shape the microenvironment ("niche") for optimal cell survival, adhesion, spreading, proliferation, migration, differentiation, as well as tissue formation and repair. ${ }^{[85]} \mathrm{ECM}$ can be harvested from a variety of allogeneic or xenogeneic tissue sources, including dermis, urinary bladder, small intestine, mesothelium, pericardium, and heart valves, and from several different species. ${ }^{[86]}$

Various studies show that such decellularized scaffolds can partly mimic the microenvironments in native tissues or organs. ${ }^{[81,87,88]}$ For example, Zheng, Himmelfarb, and co-workers found that hydrogels could be derived from the human kidney cortex. Compared with normal kidney tissue, such kidney ECM (K-ECM) hydrogels contained a majority of native matrix proteins, such as collagen IV, laminin, and heparan sulfate proteoglycan and their isoforms. ${ }^{[89]}$ Increasing evidence proved that such ECMs can provide niches that mimic their respective natural microenvironments of the body. Jin and coworkers recently reported that liver-derived ECM (L-ECM) from porcine origin could promote the differentiation of rat MSCs to hepatic cells. ${ }^{[88]}$ Moreover, they found L-ECM could provide a microenvironment that contained many protein signals, which when in contact with exogenous additives $\left(\mathrm{Mn}^{2+}\right)$, could lead to fibrosis through different integrin pathways.

Recently, it was demonstrated that dECM obtained from different tissues could serve as promising bioinks for 3D bioprinting (Figure 3A). ${ }^{63,81,90]}$ Cho and co-workers developed novel tissue-specific dECM bioinks, based on adipose, cartilage, and heart tissues. Tissuespecific cells could proliferate for at least 14 days in such tissue-specific bioinks. ${ }^{[63]}$ The dECMs were solubilized to obtain a final concentration of $3 \mathrm{w} / \mathrm{v} . \%$. The acidic solubilized 
dECM solution was then adjusted to physiological $\mathrm{pH}$ for encapsulating cells while maintaining the temperature below $10^{\circ} \mathrm{C}$. These cell-laden $\mathrm{dECM}$ bioinks could be deposited through an extrusion nozzle, and then undergo gelation at physiological temperature to maintain the generated 3D structures. Rheological studies indicated that the three types of dECM-based bioinks had shear-thinning properties resulting in good printability. In addition, the gelation kinetics affirmed that these bioinks started immediate gelation at $15{ }^{\circ} \mathrm{C}$ and formed a stable crosslinked hydrogel at $37^{\circ} \mathrm{C}$ within 30 min, avoiding the need of any harsh crosslinking conditions or gelatin additives. Cartilage-derived dECM (C-dECM), heart-derived dECM (H-dECM), and adipose-derived dECM (A-dECM) bioinks were used to 3D-bioprint respective tissue constructs (Figure 3B - 3D). The bioprinted and gelled multi-layered grids remained stable on the deposited position during a culture period without disintegration for over 14 days. In addition, Cho and co-workers managed to improve their dECM bioinks with vitamin B2-induced UV crosslinking and thermal gelation to induce solidification. ${ }^{[91]}$ They displayed differentiation of cardiac progenitor cells in a bioprinted cardiomyogenic tissue with increased cell viability and proliferation over a duration of 23 days in culture.

More recently, Kim, Cho, and co-workers successfully tackled the problem of cardiac repair. ${ }^{\left[{ }^{[2]}\right.}$ They bioprinted stem cell-laden $\mathrm{dECM}$ multi-material patches that were able to promote direct cell-to-cell interaction and differentiation (Figure 3E and 3F). The patches displayed high vascularization and tissue matrix formation after implantation into Balb/c mice indicating their functionality in vivo. They showed enhanced cardiac function, reduced cardiac hypotrophy and fibrosis, increased migration from patch structure to the diseased area, as well as neo-muscle and capillary formation (Figure 3G). Their study suggested the applicability of dECM bioinks in in vivo situations. Furthermore, Koc and co-workers demonstrated the applicability of decellularized tendons in bioprinting applications, while drastically reducing the gelation time of their bioprinted constructs in comparison to previous methods (Figure $3 \mathrm{H}$ and 3I). ${ }^{[93]}$ By applying an aspiration-extrusion method, where a dECM pre-gel obtained from bovine Achilles tendon gelled in a glass capillary and subsequently extruded, they were able to reduce the gelation time of their IECM bioink down to 6 min compared to 30 min gelation time using conventional protocols. The rapid gelation of their dECM bioink allows for the bioprinting of structures with high shape fidelity avoiding the need of a supporting structure. Encapsulated NIH/3T3 fibroblasts showed high viability as well as lineage-specific morphology.

In general, dECMs are auspicious candidate biomaterials for use as bioinks that are capable of providing optimized microenvironments conducive to the growth of specific tissues including but are not limited to cartilage, heart, adipose, and liver tissues.

\subsection{Electrical properties of bioinks}

In the last decade, 3D printing technologies have been widely employed to fabricate electronic circuits and devices. ${ }^{[94]}$ The traditional manufacturing strategies of electronic devices are complex and cost-intensive, produce high amounts of waste, and are not environmental-friendly with multi-stage processes of photolithography/electron beam lithography, metal deposition, and lift-off. Meanwhile, the huge demand in electronic 
devices market also results in a strong need for low-cost, simple, and highly efficient technologies as alternatives for device fabrication. ${ }^{[95]}$ To this end, 3D printing becomes a promising strategy to fabricate electronic devices that can easily achieve automated largescale fabrication of electronics with high resolution (down to $20 \mu \mathrm{m}$ ). ${ }^{[96]}$

By the use of a conductive bioink, 3D bioprinting can also be applied in tissue engineering and regenerative medicine for the fabrication of bioelectronics and conductive tissues, such as heart tissues and spinal cord. ${ }^{[97,98]}$ For the design of a bioink that is suitable for the fabrication of bioelectronics, it is necessary to include a conductive material into the bioink to ensure high electrical conductivity. It is well-known that higher concentrations of nanomaterials produce better conductivity at the same bioink volume. ${ }^{[97]}$ On the other hand, non-conductive materials can also significantly determine the conductivity of devices, which serve as organic polymeric stabilizers or adhesion promoters in the bioinks. Consequently, the electrical contact between conductive materials depends on these different aspects, which determines the electrical performance of bioprinted devices and tissues.

In general, the conductive material included into the bioink can be nanoparticles, polymers, or organometallic compounds. ${ }^{[99]}$ Here, we focus on conductive nanomaterial-based bioinks, such as those based on metal nanoparticles, graphene, and carbon nanotubes (CNTs). Metalbased bioinks provide good electrical conductivity based on highly conductive metals, such as gold, silver, aluminum, or copper. In recent decades, gold in particular, has been widely used in regenerative medicine and drug delivery for its superior biocompatibility. ${ }^{[100]}$ Shin, Khademhosseini, and co-workers incorporated gold nanorods into a GelMA-based bioink with the aim to bioprint cardiac tissue constructs. ${ }^{[101]}$ They demonstrated that the encapsulation of nanorods did not significantly influence the viscosity of the bioink allowing for co-encapsulation of cells with a high density into the bioink resulting in rapid bioprinting of cell-laden constructs with a cell viability of approximately $70 \%$. The encapsulation of gold nanorods caused an improved electrical propagation between cardiac cells and an advanced functionality of the bioprinted constructs. Their research demonstrated that bioprinted constructs with encapsulated gold nanostructures might offer a novel treatment for affected areas in the heart after myocardial infarction as well as applications in the engineering of electrogenic tissues such as the spinal cord, brain, or skeletal muscles.

Additional interesting components for conductive bioinks are carbon-based materials, such as graphene and CNTs. Graphene-based bioinks usually consist of pristine graphene (PG) or graphene oxide (GO). Although both materials would form suitable bioinks, the application of PG-based bioinks is strongly limited by the low dispersability of PG in aqueous media, resulting in final concentrations ranging from only $0.002-0.1 \mathrm{wt} . \%$ in the bioinks. ${ }^{[102]} \mathrm{GO}$ on the other hand, which is mainly produced by oxidation of graphene, shows excellent dispersability in water. ${ }^{[103]}$ Dispersions of stable GO in the bioinks usually achieve final concentrations ranging from $0.1-1.0 \mathrm{wt} . \%$, making it a suitable material for the use in bioprinting of conductive materials. ${ }^{[104]}$ Hersam, Shah, and co-workers have reported the use of graphene-bioinks to 3D-bioprint functional cardiac and spinal cord tissues (Figure $4 \mathrm{~A}){ }^{[105]}$ The encapsulation of graphene did not influence the printability of their bioink in a negative way, and allowed for rapid bioprinting of free-standing anisotropic microstructures (Figure 4B). The extrusion shear forces facilitated reorientation and alignment of 
microflakes along the flow direction. The gross effect was a filament microstructure with graphene flakes aligned along the fibers. In vitro biological properties were determined by statically seeding of MSCs onto the 3D-bioprinted scaffolds. They found that, over a duration of 2 weeks, graphene-PLG scaffolds supported MSC growth and proliferation. The 3D-bioprinted constructs showed high electrical conductivity, flexibility, and biocompatibility. Furthermore, they were biodegradable and neurogenically active. Recently, Hsu and co-workers were able to successfully confirm the functionality of such GO-based bioinks for the fabrication of neural tissue constructs by encapsulation of GO into a waterborne biodegradable polyurethane-based bioink. ${ }^{[106]}$ Encapsulated neural stem cells (NSCs) presented a high viability (>65\%) and functionality after bioprinting. Furthermore, they confirmed the differentiation of NSCs by an increased gene expression of neural tissue specific markers such as $\beta$-tubulin, glial fibrillary acidic protein (GFAP), and microtubuleassociated protein 2 (MAP2). Their research proved the application of GO-based bioinks in neural tissue engineering.

$\mathrm{CNT}$ is another excellent conductive nanomaterial to formulate conductive bioinks. However, the hydrophobicity of CNT limits their application in bioprinting, as they tend to rapidly aggregate in aqueous solutions making it challenging to obtain a homogenous distribution of CNTs in the bioink. ${ }^{[99]}$ Nevertheless, there are different strategies to obtain homogenous CNT bioinks based on the use of $i$ ) organic solvents without dispersants, $[107,108]$ ii) aqueous media with dispersants, ${ }^{[108,109]}$ and iii) chemical modification with a functional group. ${ }^{[108,110]}$ The production of CNT-based bioinks using organic solvents without dispersant usually results in low concentrations of CNT $\left(<0.1 \mathrm{~g} \mathrm{~L}^{-1}\right)$. To solve this problem, aqueous dispersants (e.g., Triton X-100 and sodium dodecyl sulfate [SDS]) have been used to obtain bioinks with high concentrations of CNTs. ${ }^{[111]}$ Subsequent ultrasonication is additionally applied to prepare homogeneous CNT bioinks. The typical concentration of these CNT bioinks ranged from $0.01-10 \mathrm{~g} \mathrm{~L}^{-1} \cdot{ }^{[112]}$ As an example, Khademhosseini and co-workers tackled the problem of a proper CNT dispersion using biosurfactants such as DNA, HA, and GelMA (Figure 4C and 4D). ${ }^{[113]}$ They were able to create a bioink with high conductivity and biocompatibility. Cardiac fibroblasts seeded on top of this structure displayed a high viability of $90 \%$ and performance demonstrating the potential of CNT-based bioinks in bioprinting applications (Figure 4E). In addition, CNTbased bioinks also show promising performance in 4D bioprinting as indicated by Gou, Leng, and co-workers. ${ }^{[114]}$ They coated CNT sheets with the shape-memory polymer Veriflex ${ }^{\circledR}$. After application of an electric current $(0.09 \mathrm{~A})$ they observed the bending of the sheets into a "U" shape, which could rapidly be reversed within 2 min after release of the electrical current. Their research demonstrated the suitability of CNT-based bioinks for the use in 4D bioprinting applications.

Besides the application in bioprinting tissue-like constructs, 3D-printed electronic devices based on conductive inks can also be used for various applications in the field of medicine such as fabricating smart sensors or novel conductors. For example, 3D-printed electronic devices were used for measurement and stimulation of on a Langendorff-perfused rabbit heart, including electrocardiography (ECG), Si strain gauge, inorganic light-emitting diodes (LEDs), as well as $\mathrm{pH}$ and temperature sensors. ${ }^{[115]}$ These elastic membrane-based sensors could easily achieve the multi-parameter analysis of the heart, which is difficult for 
conventional 2D sensors. Moreover, other groups developed a printed wearable sensor on the impedance changes of printed devices that could be used for the finger gesture recognition based (Figure 4F). ${ }^{[116]}$ Recently, Kim and co-workers developed an ink for 3D printing of lithium batteries based on conductive silver nanowires (AgNWs) with a proven conductivity of $10^{3} \mathrm{~S} / \mathrm{cm}$, which is 10 times higher compared to carbon-based conductors, and therefore especially applicable for batteries. ${ }^{[117]}$ By combining the AgNW ink with cellulose, they achieved the necessary mechanical properties to successfully print 3D constructs. Although their approaches are not yet applicable for tissue engineering applications, printing highly conductive materials might have high potential for applications in regenerative medicine such as the development of smart pacemakers and sensors for interfacing with engineered tissues. ${ }^{[118]}$

Summarizing, 3D bioprinting with conductive bioinks is a promising technique to provide efficient, fast, and low-cost fabrication of bioelectronics as well as the fabrication of conductive tissue constructs for the application in tissue engineering and regenerative.

\section{Cell Sources for Bioprinting}

Bioprinting is generally defined as the "use of 3D printing technology with materials that incorporate viable living cells", and therefore, it is clear that one of the essential parts of bioprinting is the cells used in the process. ${ }^{[119]}$ The combination of the chosen bioprinting technique, the designed bioink, and the cell source determines whether bioprinting of a viable construct is successful or not. On the one hand, the bioprinting technique should allow the fabrication of 3D constructs with minimum damage to cells, while the bioink should possess certain properties to provide an optimal microenvironment for the cells. On the other hand, the cells should be derived from a suitable source that matches the specific application of bioprinting.

Several sources of cells are available for bioprinting, ranging from MSCs from different origins to patient-derived cells (Figure 5A). MSCs are a widely used in bioprinting applications. Although these cells can be derived from different sources, which will be discussed later on, most commonly the term MSC refers to stem cells derived from the bone marrow. ${ }^{[120]}$ These multipotent stem cells generally differentiate into mesodermal lineages such as osteocytes, adipocytes, and chondrocytes as well as possessing the capacity to differentiate into ectodermal cells (e.g., neurocytes) and endodermal cells (e.g., hepatocytes). ${ }^{[120]}$ Due to this wide range of differentiation possibilities, MSCs are a prominent source for the fabrication of various tissue types. Święszkowski and co-workers used cell-laden bioinks consisting of either GelMA, chondroitin sulfate amino ethyl methacrylate (CS-AEMA), or MeHA emulating the natural ECM properties, in combination with MSCs to bioprint 3D cell-laden constructs. ${ }^{[121]}$ They observed high cell viability, chondrogenic differentiation, as well as high robustness of the obtained constructs, indicating the capability of their construct for the use as neocartilage. A similar approach was adopted by Cui and co-workers, who cultured MSCs on a 3D-bioprinted scaffold to investigate the importance of nuclear receptor subfamily 2 group F member 2 (NR2F2). ${ }^{[122]}$ This factor plays a crucial role in the regulation of mesoderm-derived tissues on chondrogenesis. Fisher and co-workers successfully demonstrated the application of MSCs for bone tissue engineering. ${ }^{[123]}$ They 
showed high cell spreading and survival as well as enhanced osteogenic differentiation of MSCs, supported by collagen type I in the used bioink. Khademhosseini, Annabi, and coworkers adopted MSCs to develop a large-scale bone construct with high structural fidelity, improved cell viability, and osteogenic differentiation as well as perfusable blood vessel-like microchannels within the construct. ${ }^{[124]}$ Other than MSCs derived from bone marrow, cells originating from adipose tissue, termed as adipose-derived stem cells (ASCs), are also used in bioprinting applications and have shown a high potential. ${ }^{[125]}$ Butcher and co-workers demonstrated a higher resistance of ASCs to increasing photoinitiator concentrations, which is known to negatively influence cell survival in bioprinted constructs, in comparison to aortic valve sinus smooth muscle cells (SMCs), resulting in higher cell survival. ${ }^{[126]}$ This study demonstrated the robustness of these stem cells and their strong potential for bioprinting applications. Gruene and co-workers confirmed this by using ASCs for the fabrication of 3D tissue grafts for adipogenic differentiation based on laser-assisted bioprinting. ${ }^{[127]}$

Besides MSCs and ASCs, Atala and co-workers further suggested that amniotic fluidderived stem cells (AFSCs), a relatively new source of stem cells with wider availability, are also suitable for 3D bioprinting applications. ${ }^{[128]}$ These multipotent cells possess a high capacity to differentiate into cell types from all three germ layers and can be maintained for long durations (up to approximately 250 population doublings) in vitro. ${ }^{[129]}$ The same group successfully bioprinted a complex heterogeneous tissue construct containing human AFSCs, canine SMCs, and bovine aortic endothelial cells (BAECs) using inkjet bioprinting (Figure 5B). They mixed the cell sources with $\mathrm{CaCl}_{2}$ solution as crosslinking agent and deposited the mixture in an alginate-collagen bath. The final construct showed proper differentiation of AFSCs into the osteogenic lineage supported by vascularization from BAECs (Figure 5C and 5D). In addition, for 3D bioprinting that specifically aims to generate tissues from the spinal cord or from the brain, NSCs are a broadly used source to fabricate these tissues. NSCs are multipotent stem cells that especially generate neurons and glial cells of the nervous system, ${ }^{[130]}$ facilitating bioprinting of functional nerve tissues. The topic will be discussed in a later section of this review addressing the 3D bioprinting of neural tissues.

Although the use of multipotent stem cells is widely applied and accepted in 3D bioprinting, their limitation to only differentiate into specific tissues might reduce overall capacity of bioprinting. Therefore, the use of pluripotent stem cells offers a superior cell source for bioprinting as these cells have the capacity to differentiate into almost every cell type in the human body. ${ }^{[131]}$ Sun and co-workers demonstrated the applicability of pluripotent stem cells by bioprinting mouse embryonic stem cells (ESCs) into 3D constructs. ${ }^{[132]}$ They observed high viability of cells ( $90 \%)$ and proliferation after bioprinting. Moreover, these ESCs formed uniform embryoid bodies (EBs) after 7 days of culture mimicking the early stages of embryogenesis. Their bioprinted construct might find good application in drug screening and might bring a better understanding of biological processes behind embryonic development. Shu and co-workers further showed the high performance of ESCs by incorporating them into an alginate-based hydrogel with the aim to fabricate a 3D mini-liver. [133] They successfully differentiated ESCs into hepatocyte-like cells, which they confirmed by measuring a constant albumin secretion, reaching its peak at 21 days after bioprinting with an amount of approximately $2.5 \mathrm{ng} \mathrm{mL}^{-1}$. Their work demonstrated the use of ESCs for 
the fabrication of complex organ structures to achieve regenerative medicine. Nevertheless, the use of ESCs especially in the case of human ESCs in research is raising ethical and political controversies. This is due to facts that ESCs can only be derived from the inner cell mass of a blastocyst, an early-stage pre-implantation embryo. ${ }^{[134]}$

Fortunately, with the advances in stem cell biology over the last decades, the use of pluripotent stem cells in tissue engineering is no longer limited to ESCs but has also expanded to the use of induced pluripotent stem cells (iPSCs). To this end, Chen and coworkers were able to successfully bioprint an in vitro 3D hepatic model by combining iPSCderived hepatic progenitor cells with human umbilical vascular endothelial cells (HUVECs) and ASCs in a rapid 3D bioprinting process (Figure 5E). ${ }^{[135]}$ They revealed enhanced morphological organization, expression of liver-specific genes, increased metabolic product secretion, and enhanced cytochrome P450 induction. Their model might form a crucial step towards personalized early drug screening as well as improving the knowledge of liver pathophysiology. Recently, Wallace, Crook, and co-workers described a 3D-bioprinted platform for the differentiation of iPSCs into self-organizing embryoids comprising cells of endoderm, mesoderm, and ectoderm lineages or into homogenous neural tissues containing functional migrating neurons and neuroglia. ${ }^{[136]}$ They managed to fabricate a fully $3 \mathrm{D}$ bioprinted structure that was able to support iPSC differentiation for culture as well as expansion (Figure 5F and 5G).

Besides the use of multi- or pluripotent stem cells, fully differentiated cells have also been used in bioprinting applications. Naturally, in this case, the choice of an appropriate cell source is highly dependent on and selective for anticipated tissue as these cells do not possess the ability to differentiate into diverse tissues. Yang and co-workers fabricated gradient structures to obtain constructs with high physiological similarity. ${ }^{[137]}$ They combined chondrocytes and collagen type II-containing bioinks with a gradient concentration of collagen to obtain zonal cartilage. This work is a step towards bioprinted constructs with a similar natural physiology. Another approach demonstrated the applications of bioprinting in drug screening. Nguyen and co-workers developed a 3Dbioprinted liver tissue containing patient-derived hepatocytes to investigate the effects of drug-induced liver injury. ${ }^{[138]}$ They proved that the 3D-bioprinted construct formed a better model for testing drugs compared to a 2D model when insulted by Trovafloxacin. The clear cytotoxic effect, which they observed, could not be assessed using commonly used preclinical models mainly based on in vitro monolayer cultures of primary human hepatocytes, due to a rapid loss of their functions and activity in these cultures. ${ }^{[139]}$

Another step towards the fabrication of complex tissues that mimic the physiological properties of their native counterparts is the combination of several types of cells harvested from different cell sources. As an example, Lewis and co-workers successfully created a thick $(\sim 1 \mathrm{~cm})$ and vascularized tissue construct that allowed perfusion for up to 6 weeks. ${ }^{[140]}$ This construct consisted of MSCs and human neonatal dermal fibroblasts (DNFs) combined with HUVECS (Figure 5H and 5I). This work proved that it was possible to combine different types of cells from different cell sources to obtain a complex tissue construct through bioprinting. 
It is clear that selection of the right cell source is an important step towards successful bioprinting of tissue constructs. Depending on the desired tissue types, cells should be chosen accordingly and provided with the optimal microenvironment allowing them to proliferate, spread, and if necessary, differentiate. Stem cells demonstrate a high potential to be used for the fabrication of patient-specific constructs, and in combination with gradientlike structures, complex architecture, and other relevant cell types, they together promise a great potential for the translation of 3D bioprinting into the clinics.

\section{Emerging Evolutions in Bioprinting}

To obtain biomimetic human tissues for translational applications, not only the choices of the proper bioprinting technique, bioink, and cell source are crucial, but it is also desirable to integrate these elements into newer 3D bioprinting strategies to further broaden the applications of bioprinting. Several different approaches have emerged over the past years that could potentially overcome the limitations of current bioprinting strategies, resulting in innovations that set milestones for further developments in this area. These innovations range from freeform bioprinting that addresses the challenges of gravity in conventional extrusion bioprinting and microfluidic bioprinting to obtain perfusable hollow vascular structures, to $4 \mathrm{D}$ bioprinting techniques taking bioprinting to the next dimension. In this section, we provide a detailed discussion on recent evolutions that have broadened the applications of bioprinting in achieving the fabrication of biomimetic tissue constructs.

\subsection{Bioprinting of complex architectures}

The human body consists of numerous complex structures ranging from the hepatic lobule in the liver and the delicate network of bronchi, arteries, and veins in the lung to the complex neural network in the brain. ${ }^{[141,142]}$ To achieve biomimetic tissues, mimicking the in vivo counterparts in both architecture and function is crucial. To this end, 3D bioprinting is a promising technique as it enables the design of complex structures based on computer-aided design (CAD) models as well as allows that designs to be directly digitized from medical images of the human tissues by for example, magnetic resonance imaging (MRI). ${ }^{[143]}$ Recently, several studies addressed the challenge to bioprint complex structures to mimic human tissues, which are described in detail in this subsection.

5.1.1. Freeform bioprinting-One major limitation with conventional bioprinting lies in their inadequacy to deposit discrete layers that are not mechanically supported by those underneath. ${ }^{[144]}$ As a consequence, a new class of 3D bioprinting techniques has been developed that permits direct extrusion of hydrogel bioinks into self-healing support hydrogels. This allows for the fabrication of discrete patterns in the volumetric space over relatively large scales and constructs with complex architectures. By design, either the deposited patterns in the hydrogel matrices can be sacrificially removed, leaving desired cavities in the 3D space, or the support matrices may be washed off to retain the bioprinted 3D structures. For example, Burdick and co-workers developed bioinks and support hydrogels based on supramolecular assembly through a guest-host system. ${ }^{[144]}$ Due to noncovalent bonding of the guest-host molecules, splitting of the bonds could be achieved when an external stimulus, such as shear stress (in the case of the bioinks) or deformation (in the 
case of the support matrices), was applied. However, they could rapidly self-heal upon removal of the stimulus. Especially, the supramolecular hydrogels that were prepared based on modification of HA with either adamantine (Ad, guest) or $\beta$-cyclodextrin $(\beta$-CD, host) (Ad-HA and CD-HA) displayed promising performance. The intermolecular guest-host bonds between Ad and $\beta$-CD rendered the modified HA the capability to self-assemble upon mixing, which allowed for direct embedded bioprinting of 3D tissue architectures (Figure $6 \mathrm{~A})$. Using such a technique, supramolecular filaments with varying diameters could be deposited in the support hydrogel by adjusting the nozzle size, the volume of extruded bioinks, or the physical properties of hydrogels. The guest-host writing further enabled bioprinting of discrete 3D structures not achievable by standard methods, such as the bioprinting of a spiral structure surrounding a cylinder in the center (Figure 6B - 6E). Significantly, these supramolecular hydrogels could also be designed to possess a secondary moiety that facilitates covalent crosslinking on demand to stabilize bioprinted 3D structures. The same group introduced photocrosslinkable methacrylate groups into the Ad-HA (AdMeHA) and CD-HA (CD-MeHA), which could be used as either bioinks or support matrices, to selectively stabilize one of these two components. For example, bifurcated microchannels were fabricated by extruding Ad-HA/CD-HA bioink into the Ad-MeHA/CDMeHA support matrix. UV-crosslinking of the support matrix permanently stabilized the construct and subsequent vacuum removal of the bioink formed hollow, perfusable channels. On the contrary, by using Ad-MeHA/CD-MeHA as bioink and extrusion into a noncrosslinkable support matrix, complex and crosslinkable 3D constructs were fabricated and retrieved by dissolving of the support matrix. The method was reported to exert no negative effects on embedded cells.

Similarly, Feinberg and co-workers used a suspension of gelatin microparticles with shearthinning properties as a support matrix to enable direct deposition of bioinks in complex 3D structures. ${ }^{[145]}$ After bioprinting, the thermosensitive gelatin support matrix could be selectively dissolved by elevating the temperature to $37{ }^{\circ} \mathrm{C}$ to retrieve bioprinted biomimetic tissues. With this technique, they were able to produce complex structures ranging from vascular and bone constructs to brain and heart models (Figure 6F - 6I). Angelini and coworkers adopted a similar idea by using a soft granular gel made from micrometer-sized soft hydrogel particles as support matrix that smoothly transited between fluid- and solid-like states. ${ }^{[146]}$ The medium was fluidized under locally applied low shear stresses permitting direct writing of the bioinks, and rapidly re-solidified when the stresses were released, allowing for continuous bioprinting of complex 3D shapes (Figure 6J and 6K). Through subsequent removal of the support hydrogel the constructs could be retrieved after bioprinting. In a recent approach, Huang and co-workers took advantage of the physical crosslinking mechanism of alginate by $\mathrm{Ca}^{2+} \cdot{ }^{2147]}$ They designed an approach to fabricate $3 \mathrm{D}$ alginate structures based on a granular hydrogel support material-enabled two-step gelation process. The first step was based on the extrusion of an alginate-gelatin bioink into a 0.8 w/v.\% Carbopol hydrogel environment, which allowed the freeform fabrication of constructs without the necessity to immediately crosslink the bioink. This step avoided clogging of the nozzle as well as allowing the different layers to easily fuse into a single construct. Gelatin in the bioink served as a sacrificial layer, which was thermally gelled after extrusion into the Carbopol environment (first gelation process). After removal from the Carbopol 
environment, gelatin was melted and diffused away while alginate was crosslinked in a $\mathrm{CaCl}_{2}$ bath (second gelation process).

5.1.2. Stereolithographic bioprinting-While conventional stereolithography typically suffers from the use of static patterns and low bioprinting speeds, in recent years this technique has undergone significant developments towards its use for rapid, dynamic patterning of biomaterials to achieve constructs with complex architecture. The dynamic photopatterning in stereolithography can be achieved by using a digital micromirror device (DMD). A DMD consists of millions of micro-sized mirrors, which can be individually tilted to allow for the formation of controllable patterns during the photocrosslinking processes of consecutive layers, thus enabling rapid fabrication of complex 3D structures. Chen, Khademhosseini, and co-workers observed enhanced cell proliferation and uniform cell distribution of HUVECs in DMD-fabricated GelMA scaffolds (Figure 7A). ${ }^{\text {[148] }}$ Furthermore, they showed that the cells maintained their endothelial phenotype, demonstrating the biological functionality of the scaffolds using DMD-based stereolithography bioprinting. At the same time, Chen, Zhang, and co-workers verified the performance of this method by the rapid fabrication of microwells of 150-200 $\mu \mathrm{m}$ in diameter with different surface topographies to study the interactions between cells and their microenvironment. ${ }^{[149]}$ They demonstrated different geometric guidance of HUVECs and NIH/3T3 fibroblasts with bioprinted scaffolds containing 15 w/v.\% GelMA and 20 w/v.\% PEGDMA (Figure 7B). Zhang and co-workers bioprinted nanocomposite matrices in different shapes to investigate bone metastasis of breast cancer (Figure 7C). ${ }^{[150]}$ They compared MDA-MB-231 breast cancer cells with a high potential of metastasis with MCF-7 breast cancer cells possessing a low capacity of metastasis, in a PEG-diacrylate (PEGDA)based bioink embedded with hydroxyapatite nanoparticles to mimic the human bone. They successfully created a 3D platform to investigate bone metastasis of breast cancer cells with an improved performance compared to 2D cultures.

Cell-laden hydrogels have also been recently used to fabricate biological structures with DMD stereolithography bioprinters. Chen and co-workers proved this concept by bioprinting cell-laden GelMA constructs (Figure 7D). ${ }^{[151]}$ They also presented the suitability of stereolithography as a platform to study cell interaction by creating complex micro-features in a short amount of time and eventually achieving high-throughput screening platforms. Yang and co-workers combined the process of stereolithography and extrusion-based bioprinting in a "hybprinter", which was able to create scaffolds with rigid and soft components made from PCL and cell-laden PEGDA. ${ }^{[152]}$ Viability studies with HUVECs displayed a high cell viability after bioprinting of a conduit structure (Figure 7E), exhibiting the capacity of this bioprinting system for tissue engineering applications.

Most recent trends in stereolithography-based systems have focused on creating highly complex structures by combining different approaches or further develop the process of stereolithography to reach very high resolutions. Bashir and co-workers described the combination of dielectrophoresis (DEP) with stereolithography for the spatial patterning of cells on custom-made gold microelectrodes followed by encapsulation of mouse ESCs and C2C12 skeletal muscle cells in PEGDA hydrogels of different stiffness (Figure 8A). [153] They demonstrated the enhanced biomimetic properties of their 3D microtissue 
architectures. This technique showed strong potential in applications in tissue engineering focused on stem cell differentiation. For example, the same group successfully developed a biological robot based on stereolithography that mimicked the structure and architecture of skeletal muscle proving the capability of stereolithography to crate biomimetic tissue constructs. The robot was powered by contraction of an engineered mammalian skeletal muscle strip to characterize the influence of different ECM proteins (collagen I and fibrin) as well as insulin growth factor I on the force production of the muscle (Figure 8B and 8C). ${ }^{[154]}$ They have successfully proven that they were able to measure the force and velocity of the contraction after applying an electrical stimulus to the muscle strip. Their work demonstrated the possibility to successfully combine cells and tissue with engineered robotic devices in tissue engineering applications. In another work Bashir and co-workers reported a new micro-stereolithography-based apparatus ( $\mu \mathrm{SLA}$ ) that reached a resolution of $<5 \mu \mathrm{m}$ and was capable of bioprinting multiple materials (Figure 8D). ${ }^{[28]}$ They again demonstrated the performance of their bioprinter for further application in tissue engineering by incorporation of different cells (fibroblasts, myoblasts, endothelial cells, and MSCs) into a PEGDA hydrogel resulting in high cell viability after bioprinting. This innovative bioprinter was also capable of depositing multiple materials allowing the fabrication of a cellencapsulating patch designed to promote targeted growth of neovasculature. Their bioprinting system might provide a suitable tool for studying cell-ECM interactions, highthroughput drug testing, and programmable tissue engineering for applications in regenerative medicine.

More recently, Lim, Woodfield, and co-workers developed a novel bioink to fabricate highly complex architectures through stereolithography. ${ }^{[155]}$ By combining methacrylated PVA (PVA-MA) and GelMA together with a transition metal-based visible light photoinitiator, they were able to achieve biologically relevant constructs with a high spatial resolution of 25 $\mu \mathrm{m}$. Delicate architectures from woven mats, chain nail designs, or 3D lattice structures to complex gyroid structures could be bioprinted (Figure 8E). MSCs, isolated from human patients and equine articular cartilage-derived progenitor cells (ACPCs) encapsulated into the bioink showed high viability ( 90\%) after 14 days of culture as well as osteogenic differentiation of seeded MSCs and cartilage-specific ECM deposition of ACPCs, proving the application of this bioink/photoinitiator combination for bone and cartilage tissue engineering.

Besides recent trends using stereolithography-based bioprinting in achieving highly complex cell-laden constructs for the fabrication of complex biomimetic tissues, stereolithography can also be applied in other related fields of biomedical research. Back in 2014, Chen, Gou, and co-workers demonstrated the application of stereolithography in toxicology. ${ }^{[156]}$ They bioprinted a liver-inspired 3D construct with encapsulated polydiacetylene nanoparticles that were able to attract and capture toxins while the 3D architecture efficiently trapped the toxins within the construct. A high performance of their biomimetic detoxification device was observed, proving the capability of 3D bioprinting for applications in toxicology. In 2015, the same group of Chen, Wang, and co-workers continued this line of work by developing functional microswimmers.(Figure 8F). ${ }^{[157]}$ These microswimmers exhibited chemically powered and magnetically guided propulsion by incorporation of platinum and iron oxide nanoparticles, as well as efficient detoxification capabilities, proven by the 
incorporation of polydiacetylene (PDA) nanoparticles. Using a custom-modified DMD bioprinter, they achieved a resolution of $1 \mu \mathrm{m}$ with a high bioprinting speed demonstrating the potential of rapid high-resolution stereolithography bioprinting.

Besides these endeavors that focus on the application of stereolithography in biomedicine, several studies have reported the development of emerging techniques to improve the current stereolithography bioprinting platforms. A general field of interest is development of fast bioprinting procedures. In fact, stereolithography forms a good basis to enhance the speed of 3D manufacturing. DeSimone, Ermoshkin, Samulski, and co-workers have developed a continuous liquid interface production (CLIP), which allowed printing of objects with complex architecture in a very short period of time. ${ }^{[158]}$ This platform made printing objects much faster in comparison to any existing stereolithography-based platforms by adopting an oxygen-permeable window below the projection plane forming a persistent liquid interface referred to as the "dead zone". Zambelli and co-workers developed a technique for additive manufacturing of metals to create complex shapes and structures. ${ }^{[159]}$ A microchanneled atomic force microscopy (AFM) with an aperture at the tip apex was used for the controlled deposition of droplets, eventually achieving high-resolution 3D structures. Although methods such as CLIP or AFM-based 3D printing are not yet suitable for the integration of cells, the technological innovations might have significant influence on the development of novel strategies for rapid 3D bioprinting in the future.

In summary, current bioprinting systems are already able to achieve complex tissues at high resolutions, which is crucial for the formation of biological tissues. Although not all systems mentioned in this section are already capable of incorporating cells into the bioprinting process, the aim to properly mimic human tissues have resulted in innovative and promising bioprinting techniques and platforms. Further research on these systems for the incorporation of cells will have a significant influence on the fabrication of biomimetic tissues.

\subsection{High-resolution bioprinting}

Similar to speed, the development of bioprinted tissues with complex architecture to mimic human structures has also required a constant improvement in the resolution that can be obtained from the bioprinting systems. In particular, the resolution of a bioprinting system can be a crucial limitation when producing complex in vivo-like fine architectures that are typically not larger than a few micrometers. ${ }^{[160]}$

For conventional extrusion-based bioprinting, the resolution is mostly limited by the dimension of the printhead, which can vary from a few micrometers to millimeters. ${ }^{[23]}$ For example, Lewis, Nuzzo and co-workers bioprinted poly(2-hydroxyethyl methacrylate) (pHEMA) scaffolds at a resolution of approximately $10 \mu \mathrm{m}$ using a $10-\mu \mathrm{m}$ glass microcapillary nozzle (Figure 9A), which, to the best of our knowledge, has been the highest resolution reported using extrusion bioprinting so far. ${ }^{[161]}$ To achieve higher resolutions of bioprinting, electrospinning has attracted the attention of the field (see Section 2.5 for more details on the technology). ${ }^{[162]}$ One of the unique properties of electrospun fibers is their high specific surface areas attributing to their small diameters. The thinnest electrospun fibers can be achieved at $1.6 \mathrm{~nm}$ or less in diameter. ${ }^{[162,163]}$ As a result, EBB was developed 
by combining electrospinning with an $\mathrm{X}-\mathrm{Y}-\mathrm{Z}$ robotic stage, which is capable to bioprint structures in micro- to nanometer scales. ${ }^{[46,48,63,164,165]}$ One key challenge in EBB is to overcome the bending instability and realize the controllable deposition of electrospun fibers. Besides using a shorter collecting distance, the success of EBB is also influenced by other parameters such as flow rate, moving speed of the nozzle/collector, and voltage.

Lin and co-workers used NFES to bioprint well-controlled nanofiber patterns utilizing a stable liquid jet region with a collecting distance from $500 \mu \mathrm{m}$ to $3 \mathrm{~mm} .{ }^{[46]}$ The bioprinted patterns consisted of nanofibers with a diameter of $300 \mathrm{~nm}$. Madou and co-workers further developed NFES to continuously bioprint controllable patterns in larger scales. ${ }^{[47,165]}$ Furthermore, Park and co-workers demonstrated EBB as a versatile method to bioprint various structures with a resolution of $700 \mathrm{~nm}$ using multiple functional bioinks. ${ }^{[48]}$ They were also able to bioprint more complex structures including the letter "E", 3D square structures of anthracene, and an alphabetic pattern spelling "UNIST" (Figure 9B). Although these strategies haven proven that EBB can be used to bioprint well-defined 3D constructs with high resolution, they have not been evaluated for their potential to be used for cell culture.

Recently, extensive efforts have been devoted to further developing MEBB strategies with particular focus on the bioprinting of PCL, as this polymer has proven excellent performance to serve as scaffolding material for cell culture. ${ }^{[50,51,164,166]}$ Typically, in MEBB straight fibers can be bioprinted at a faster moving speed of the collector (Figure 9C). With the optimized parameters, PCL microfibers were bioprinted in a highly controllable manner making MEBB highly promising for the fabrication of high-resolution constructs for applications in tissue engineering and regenerative medicine (Figure 9D - 9G). ${ }^{[51,80]}$

However, due to high temperatures during the melting process as well as the high voltage used for extrusion of the fibers, it is almost impossible to directly bioprint cell-laden constructs. Nevertheless, due to the high-resolution of this method it is possible to create well-defined scaffolds that allow for post-seeded cells to enter and form cellularized tissues. Recently, Hutmacher, Dalton, and co-workers demonstrated that PCL scaffolds could be bioprinted by MEBB that displayed enhanced fibroblast penetration attributing to their high porosity and interconnectivity. ${ }^{[51]}$ After 7 days of in vitro culture, the seeded fibroblasts exhibited an elongated morphology. After 14 days of culture the scaffolds were fully covered by cells (Figure 9H). Immunohistochemical analysis further confirmed homogeneous distribution of cells, as well as the expressions of ECM proteins, that are typically produced by fibroblasts, such as collagen type I and fibronectin, throughout the entire constructs (Figure 9I). Furthermore, Malda, Hutmacher, and co-workers reported MEBB of PCL constructs for the reinforcement of GelMA hydrogels aiming to regenerate cartilage tissue (Figure 9J). ${ }^{[80]}$ They were able to show a high viability of seeded chondrocytes $(>80 \%)$ after 1 day of in vitro culture. The viability remained at approximately $80 \%$ after 7 days of culture. In addition, they systematically studied the mechanical properties of the hydrogels after reinforcement. The stiffness of these PCL scaffolds was mostly depending on their porosity. A scaffold with approximately $93 \%$ of porosity showed the highest stiffness of close to $15 \mathrm{kPa}$. After reinforcement with PCL, the stiffness of the GelMA structures displayed a 30 -fold increase from $7.1 \pm 0.5 \mathrm{kPa}$ to $214 \pm 24 \mathrm{kPa}$. The reinforced hydrogels also 
possessed good elastic property even after 20 cycles of continuous $20 \%$ strain. They further proved that the PCL-reinforced hydrogels could be tailored to own a competitive stiffness and deformation profile similar to that of native cartilage (Figure 9K). ${ }^{[167]}$ Recently, the same group demonstrated that melt-electrospinning writing (MEW), a variation of MEBB, can achieve high-volume scaffolds of up to 7-mm height with uniform morphology and fiber diameters. ${ }^{[168]}$ They designed a MEW system, that automatically adjusted the collector distance according to the build height of the constructs, combined with a simulation-based increase of the applied voltage. In this way, they were able to print large volume scaffolds with well-defined architecture. Additional in vitro experiments using human periodontal ligament (PDL) cells and MSCs proved the suitability of their scaffolds for the application in tissue engineering and regenerative medicine. Their system demonstrates an approach to exceed the current height limitations of MEBB systems, which are often limited to 2-3 mm, facilitating the fabrication of large scale tissue constructs with well-defined architecture and high resolution.

\subsection{Bioprinting of gradient and multi-material structures}

Some tissues in the body present pronounced gradients in ECM composition and/or cell population, particularly those at interfaces such as the osteochondral interface, ${ }^{[169]}$ the ligament-to-bone interface, ${ }^{[170]}$ and the tendon-to-bone insertion site. ${ }^{[171]}$ Conventional methods rely on the use of anisotropic scaffolds containing distinctive or continual sections with properties matching those in the native tissues to be engineered. ${ }^{[172]}$ Despite success, precise reproduction of continuous gradients in the chemical composition, material properties, or cell types have hardly been achieved before, due to limitations associated with manufacturing. In fact, the continuous extrusion process for bioprinting has provided an excellent basis to realize alike gradients in 3D volumes, when combined with a multimaterial microfluidic nozzle platform.

By incorporating a rotating impeller into the central channel of an advanced two-way microfluidic printhead, Lewis and co-workers were able to achieve rapid and thorough mixing of two bioinks upon contact before extruding the microfibers to form bioprinted structures (Figure 10A) ${ }^{[173]}$ It was possible to fabricate 3D constructs or 2D patterns with continuous gradients of encapsulated chemicals and/or material properties by carefully tuning the volume ratio of the two bioinks (Figure 10B). Khademhosseini, Dentini, and coworkers similarly developed a microfluidic printhead for extrusion bioprinting of lowviscosity bioinks (Figure 10C). ${ }^{[69]}$ Specifically for this application, they optimized a composite bioink composed of GelMA and alginate, which in a first step could be physically crosslinked for the alginate component during the extrusion process to render structural stability of the constructs. While the GelMA component could be subsequently chemically crosslinked in the second step by UV illumination to permanently fix the shape of the tissues. The alginate present in the bioprinted structures might be removed using a $\mathrm{Ca}^{2+}$ chelating agent such as ethylenediaminetetraacetic acid (EDTA) to minimize its negative effects on the stretching and proliferation of embedded cells. Alternative or simultaneous deposition of the bioinks using this microfluidic printhead allowed for convenient fabrication of 3D multi-component microfibrous tissue constructs of interest (Figure 10D 10F). 
On top of the microfluidic printhead designs, Zhang, Khademhosseini, and co-workers further designed a continuous multi-material 3D extrusion bioprinting strategy by development of a platform based on the extrusion of 7 different bioinks through a single nozzle that was significantly faster compared to other multi-material approaches (Figure $11 \mathrm{~A}) .{ }^{[174]}$ With this platform, they bioprinted multi-component constructs with a single-step bioprinting process (Figure 11B and 11C). Furthermore, constructs containing multiple cell types displayed high cell viability and proliferation. Moreover, they rapidly extruded gradient structures of GelMA including different concentrations of hydroxyapatite or nanosilicate to display a gradient of attachment and proliferation of MC3T3 preosteoblasts seeded on top of these constructs (Figure 11D). They could also bioprint a gradient structure of conductive bioinks consisting of GelMA and different concentrations of CNT demonstrating the possibilities for bioprinting bioelectronic devices (Figure 11E).

Although the most favored bioprinting strategy to deposit multiple materials in a single construct is perhaps extrusion-based bioprinting, several innovations have also focused on the application of stereolithography in the bioprinting of multiple materials in a single process. ${ }^{[175,176]}$ These studies mainly aimed to design new stereolithographic bioprinters that are not limited to one static bioink reservoir, but would allow for the rapid exchange between different materials during the bioprinting process. To this end, Wicker and coworkers developed new multi-material stereolithography (MMSL) system that was based on a commercially available printer equipped with a reservoir-leveling system, combined with a standalone rotating stage. ${ }^{[177]}$ Chen and co-workers demonstrated a proof-of-concept rotation table that allowed the exchange of two different materials. ${ }^{[178]}$ The rotating table held two resin vats, two brush tanks, an ultrasound cleaner, and a fan. These parts would sequentially pass through the printing area where UV polymerization occurred. Chen and co-workers proposed another mechanism to switch materials. ${ }^{[179]}$ They designed and fabricated an inlet underneath the resin reservoir that held different hydrogel monomer solutions. After bioprinting, the first material could be washed out of the reservoir and the second material could be purged from the inlet into the reservoir and then aspirated by a syringe pump. This work has recently been expanded by Khademhosseini, Zhang, and coworkers, by inclusion of an automated microfluidic chamber device, to the bioprinting of multiple cell-laden bioinks in a single process (Figure 11F). ${ }^{[180]}$ Combining this technique with a DMD enabling rapid dynamic patterning, they were able to obtain complex multimaterial tissue constructs with high spatial resolution (Figure 11G - 11J). Further preliminary in vivo experiments using bioprinted structures consisting of a PEGDA frame with vascular endothelial growth factor (VEGF)-enriched GelMA layers inside, displayed neoangiogenic properties in rats demonstrating the capability of their system to bioprint biocompatible constructs. This platform opens up a previously unavailable strategy to bioprint multi-material cell-laden constructs with very high spatial resolution and might be a significant step towards the bioprinting of fully functional and biologically relevant tissues for regenerative medicine.

In summary, the trend towards 3D bioprinting with multiple materials allows for the exact deposition of multi-component or multi-cellular structures within a single construct. The bioprinting of gradient structures is hereby of particular interest of tissue engineers, as it is crucial for mimicking the structure and function of biologically relevant tissues. 


\subsection{In situ bioprinting}

Besides conventional bioprinting approaches, which are usually based on the bioprinting of tissue constructs that are subsequently used for implantation, in situ bioprinting offers a new strategy that enables deposition of the tissue right at the site of need. ${ }^{[181]}$ In general, in situ bioprinting relies on the placement of a (porous) tissue analogue in the defect that can engraft with the surrounding endogenous tissue resulting in neovascularization into the tissue analogue and integration of the analogue in the surrounding. In 2007, Campbell and Weiss introduced the concept of in situ bioprinting; however, back then it was considered challenging due to the fact that clinicians prefer simple off-the-shelf solutions instead of complex bioprinting devices. ${ }^{[182]}$ To overcome this hurdle, several improved and simplified bioprinting systems have been since developed to make in situ bioprinting available to the broader audience to facilitate its translation into the clinics. ${ }^{[183]}$

One of the major disadvantages of current bioprinting systems, is the need for flat surface to either deposit the material onto in the case of most extrusion- or inkjet-based systems, or a planar layer to crosslink the material in the case of stereolithography (see Section 2 for a detailed description on different bioprinting methods). However, defects in many tissues or wounds in the skin for example, often display a heterogenous surface topology, ${ }^{[184]}$ making it challenging for conventional bioprinting techniques to be used in the treatment of such defects. Back in 2012, Soker and co-workers introduced the concept of in situ bioprinting directly onto larger skin wounds using an extrusion bioprinting device in combination with a laser, which was used to scan the surface topology and position the printhead accordingly (Figure 12A). ${ }^{[185]}$ They bioprinted AFSCs and MSCs suspended in a fibrin-collagen matrix onto a $2.0 \times 2.0-\mathrm{cm}^{2}$ full-thickness skin wound located in the mid-dorsal region of a mouse. They found that AFSCs and MSCs in the hydrogel matrix had a beneficial effect on wound closure and re-epithelization featuring an increased microvessel density within the bioprinted tissue (Figure 12B). However, they did not observe a full integration of AFSCs and MSCs with the surrounding endogenous tissue, taking the conclusion that a full integration might heavily rely on secreted factors rather than direct cell-to-cell contact. Recently, the same group of Skardal, Soker, and co-workers supported their previous observation by developing a novel photocrosslinkable heparin-conjugated HA (HA-HP) hydrogel that prolonged growth factor and cytokine release of AFSCs within the matrix. ${ }^{[186]}$ After bioprinting their HA-HP hydrogel onto a full-thickness skin wound using the earlier described in situ bioprinting system, they observed increased wound healing through reepithelization as well as increase ECM production (Figure 12C). Through this series of studies, they have been able to prove how in situ bioprinting can be used in the healing of severe skin wounds and demonstrated how bioinks can be directly bioprinted onto wounds using a laser-assisted bioprinting system. A similar bioprinting system was recently used by Wang, Jiang, and co-workers for the treatment of large-scale bone and cartilage defects. ${ }^{[187]}$ They used a high-resolution 3D scanner to map the exact shape of large segmental defect of long bones, free-form fracture of femoral condyle and a grade-IV chondral lesion, followed by extrusion bioprinting of alginate and MeHA directly into the defect and subsequent crosslinking of the hydrogels (Figure 12D and 12E). Although this approach did not yet include cells into the bioprinting process, their study demonstrated how in situ bioprinting systems could also be applied for the treatment of large-scale bone and cartilage defects. 
A different strategy for in situ bioprinting, which has already shown promising translation to the clinics, is the use of handheld bioprinting devices. ${ }^{[183,188]}$ Di Bella and co-workers developed a handheld 3D bioprinting pen (biopen) for the treatment of articular cartilage damage (Figure 13A). ${ }^{[189]}$ The biopen was designed to simultaneously extrude a bioscaffold consisting of GelMA-MeHA and MSCs directly into a cartilage defect. In vivo experiments in sheep with an induced critical-size chondral defect revealed enhanced cartilage regeneration after treatment with the biopen proven by microscopic, macroscopic, and biomechanical analyses (Figure 13B). This approach demonstrated the potential of bioprinting to be directly applied at the location of need to treat severe chondral defect. In addition, Amini-Nik, Jeschke, Günther, and co-workers reported a handheld skin bioprinter that was able to deposit consistent sheets onto severe skin wounds (Figure 13C). ${ }^{[190]}$ By embedding dermal and epidermal cells into different crosslinkable hydrogels containing alginate or fibrin mixed with collagen type I and HA, they were able to produce skin cellladen sheets with controllable thickness, width, and composition. Application of these skin sheets onto the skin wound in a murine and porcine model demonstrated normal wound healing by re-epithelization and wound contraction (Figure 13D). Their handheld skin bioprinter showed promising capability to be used in large-scale skin wounds. More recently, Jang, Khademhosseini, Zhang, and co-workers developed a similar handheld system to inject silicate-based shear-thinning hydrogels (STHs) encapsulating vasculogenic growth factor-loaded PCL nanoparticles (Figure 13E and 13F). ${ }^{[191]}$ Through deposition using a multi-material, single-nozzle printhead, they were able to bioprint different STHs directly into a bone defect (Figure 13G). Encapsulated osteogenic and endothelial cells into the STHs indicated enhanced osteogenic differentiation as well as neovascularization. Their work showed how handheld bioprinted devices could also be used for the treatment of bone defects by combining tissue-specific cells with native growth factors to enhance tissue regeneration.

In conclusion, in situ bioprinting devices possess a high potential to treat defects in skin, bone, and cartilage by using the endogenous surrounding tissues to integrate deposited bioinks and to regenerate damaged tissues. Especially the use of handheld devices has high potential to be translated into the clinics due to easy application, which might be preferred by clinicians.

\subsection{D bioprinting}

Despite the recent advances in replicating the complex architecture and composition of human tissues, 3D bioprinted tissue constructs often remain a closed and static model of the realistic situation. However, the unique functions of natural human tissues are often based on dynamic changes in the tissue conformation commonly induced by external stimuli. ${ }^{[141,192]}$ For instance, the rhythmic pumping of the heart regulating blood circulation throughout the body, is governed by the parasympathetic nervous system and actuated by electric stimuli causing the cardiac muscles to contract. ${ }^{[141]}$ Similarly contraction of the musculoskeletal system based on electric stimuli from the nervous system facilitates movement of the body allowing us to walk and act. ${ }^{[141]}$ Besides actuated by electric stimuli, conformation of tissues can also be caused by chemical cues or hormones. For example, the hormone epinephrine (adrenalin) or the release of nitric oxide causes vasodilation resulting in an 
increased blood flow and often the result of an unbalance in the body homeostasis. ${ }^{[193]}$ To mimic these actions, it is necessary to fabricate tissues that can respond to different stimuli. In essence, $4 \mathrm{D}$ bioprinting is a new trend originating from $3 \mathrm{D}$ bioprinting but is supplemented with a new fourth dimension of time besides the 3D space coordinates. [194-196] In other words, in 4D bioprinting, the 3D-bioprinted construct can change its shape or structure with time under external stimuli in a programmed manner. ${ }^{[197]}$ The stimuli may include water, $\mathrm{pH}$, temperature, electricity, ionic strength, light, magnetic field, pressure, acoustic wave, and their combinations. Common strategies to generate adaptive structures in 4D bioprinting are the self-assembly of elements, bi-stability, deformation mismatch, and the shape memory effect. ${ }^{[198]}$ The promising applications of 4D bioprinting have spanned a wide range from biorobotics, drug delivery, and biomedical devices to tissue engineering. $[199,200,201]$

Tibbits and co-workers first reported the design of 4D bioprinting by demonstrating the selffolding of a 2D letter spelling "MIT" and a 3D cube from a one-dimensional (1D) strand, utilizing two types of materials with different water-absorbing capabilities. ${ }^{[194]}$ Dunn and co-workers bioprinted thermomechanically programmable composites using glassy shape memory polymer fibers. ${ }^{[195,202]}$ They presented complex structures including bent, coiled, twisted strips, folded shapes, and complex contoured shapes with non-uniform, spatially varying curvature. The shape of these structures was reversible in response to different temperature. Spinks and co-workers demonstrated that 4D bioprinting can be used to fabricate thermally actuating hydrogels with robust mechanical property using an alginate/ poly ( $N$-isopropyl acrylamide) (PNIPAAm) ionic covalent entangled (ICE) hydrogel for the control of water flow. ${ }^{[201]}$

Interestingly, Lewis and co-workers reported a biomimetic 4D bioprinting strategy using cellulose fibril/acrylamide hydrogel composite. ${ }^{[203]}$ The shear-induced alignment of cellulose fibers could be achieved during the process of bioprinting. The aligned cellulose fibrils could then lead to an anisotropic stiffness of bioprinted filaments and a four-fold difference of transverse and longitudinal swelling strain of $10 \%$ and $40 \%$, respectively (Figure 14A). By taking advantage of this swelling behavior and by using a predictive model, the complex structure of a calla lily flower was designed and bioprinted. The bioprinted structure showed a good agreement with the predicted model. They also mimicked the orchid Dendrobium helix containing bending, twisting, and ruffling corolla structures (Figure 14B and 14C). More recently, Zhang and co-workers developed a renewable soybean oil-epoxidized acrylate material for 3D bioprinting that possessed shapememory properties. ${ }^{[200]}$ By using a stereolithography-based bioprinting approach, they bioprinted a scaffold that was capable of supporting the adhesion and growth of seeded MSCs onto the scaffold. Additionally, they could fix their material into a temporary shape at $18{ }^{\circ} \mathrm{C}$, which fully recovered to their original shape at $37^{\circ} \mathrm{C}$ proving its suitability for $4 \mathrm{D}$ bioprinting applications.

Another approach of 4D bioprinting was recently reported by Zhao and co-workers, who used a magnetic field to control the shape of their bioprinted constructs. ${ }^{[204]}$ Ferromagnetic neodymium-iron-boron microparticles and silica nanoparticles were incorporated in their bioink for direct writing (Figure 14D). By applying a magnetic field during the extrusion of 
the bioink, they created 3D-bioprinted constructs with precise ferromagnetic domains allowing for the exact control of the constructs shape upon further stimulation (Figure 14E $14 \mathrm{H})$. Although their approach does not yet include cells in the process, the shape control of bioprinted constructs via magnetic stimulation offers a novel and highly promising method for $4 \mathrm{D}$ bioprinting. A similar approach of magnetic field stimulation was demonstrated by Duarte Campos and co-workers, who used a magnetic field to align collagen fibers within a bioprinted tissue. ${ }^{[205]}$ They incorporated streptavidin-coated iron nanoparticles in a bioink of agarose and type I collagen. By applying a magnetic stimulus during the extrusion of the bioink, it was possible to align these collagen fibers, resulting in tissues with up to $20 \%$ higher compression moduli compared to the unaligned collagen fibers. Experiments including primary human knee articular chondrocytes showed that magnetic alignment on collagen fibers had no negative effects on cell viability but increased the production of cartilage specific ECM components such as collagen type II. They demonstrated that magnetic field stimulation could be applied to manipulate bioinks in such ways to increase tissue-specific cell response without influencing cell viability.

In conclusion, 4D bioprinting may open a new way for 3D bioprinting towards various applications and with the development of smart biomaterials and smart design, new strategies to stimulate the transformation of 3D structures is around the corner. For example, 4D-bioprinted smart biorobotics can be used for disease diagnosis or drug delivery with programmable release. In addition, smart tissue constructs might be realized by this concept for better regeneration of functional tissues and organs.

\section{Bioprinting of Functional Tissues}

The recent developments in bioprinting have allowed the potential in transitioning from proof-of-concept systems towards clinical applications. The concept of on-demand bioprinted tissues, such as blood vessels and bone/cartilage to complete organs has become a promising solution to the shortage of donor organs in regenerative medicine. The translation of 3D bioprinting towards clinical use is one of the most ambitious aims for research in this field. ${ }^{[27,133,206]}$ While the previous sections have described different strategies and components that are crucial for the bioprinting of biomimetic tissues as well as the recent developments in this field, this section will focus on the application of 3D bioprinting in tissue biofabrication. The demonstration of bioprinted structures in a realistic biological environment is a crucial step to prove their biocompatibility, stability, and performance when exposed to biological conditions and to eventually enable the clinical translation of 3D bioprinting. Of particular interest in this section are studies that have shown great capability to reach in vivo investigations in the near future and/or have already included the implantation of 3D-bioprinted constructs into animal models.

\subsection{Vascularized tissues and standalone vascular structures}

Blood vessels are an indispensable component in maintaining the viability and functionality of engineered tissues and organs through their unique capacity in providing sufficient transport of oxygen and nutrients within the tissues. ${ }^{[7,12,207]}$ Many tissue engineering strategies have been devised to promote the vascularization capacity of biomaterials and/or 
tissue constructs, including for example, the incorporation of angiogenic growth factors such as VEGF, basic fibroblast growth factor (bFGF), and platelet-derived growth factor (PDGF), co-culture with pericytes and SMCs, as well as hypoxia induction. ${ }^{[208]}$ These various approaches have led to great success, particularly when the formations of microvascular networks are involved. However, the capability to generate perfusable pre-vascularized tissues remains inherently challenging.

To this end, sacrificial bioprinting technologies developed in the past few years have provided a convenient solution for this problem due to its capability to fabricate interconnected microchannels of arbitrary geometries and connectivity. ${ }^{[175,209-211]}$ Sacrificial bioprinting features four successive steps: $i$ ) deposition of the sacrificial microfibrous bioink that will eventually become the microchannels; ii) casting of the hydrogel embedded with cells over the templating microfibers to construct the tissue block; iii) removal of the template through dissolution, temperature-induced phase transition, or mechanical extraction; and iv) endothelialization of the microchannels with infused endothelial cells. A suitable sacrificial bioink should possess properties such as high biocompatibility, meaning that the bioink should not exert cytotoxicity during deposition and encapsulation processes. Furthermore, the sacrificial bioink should be removable in the presence of cells, requiring that the conditions under which the bioink is removed should not affect the behavior of encapsulated cells in the bulk hydrogel.

Thus, several main categories of sacrificial bioinks have been developed based on these requirements. For example, Chen and co-workers bioprinted microfibrous scaffolds using carbohydrate glass. ${ }^{[168]}$ These microfibers were mechanically stable to support the subsequent casting and gelation of the matrix surrounding them, but could be rapidly dissolved when the construct was immersed in culture medium. Lewis and co-workers found that Pluronic F127 solution with a certain concentration range behaved as a shear-thinning hydrogel at elevated temperatures while liquefying when temperatures approached $0{ }^{\circ} \mathrm{C}$ (Figure 15A). ${ }^{[175]}$ Such a property allowed for maintenance of the cylindrical shape of the bioprinted bioink, the embedding process at room temperature, and the possibility to wash out the liquefied sacrificial bioink by lowering the temperature. Using a combination of Pluronic F127 as sacrificial bioink and GelMA as the hydrogel matrix, multi-layer vascularized tissue structures could be constructed (Figure 15B). The same approach was followed by Mi and co-workers to fabricate multi-layered blood vessels based on AECM obtained from porcine cartilage. Using Pluronic F127 as sacrificial material they indicated bioprinting of blood vessels with a smallest diameter of $500 \mu \mathrm{m}$ embedded in a dECM-based tissue-like construct containing MDA-MB-231 breast cancer cells. After seeding with HUVECs they obtained endothelial-coated vessels within the construct. ${ }^{[212]}$ Similarly, Dai and co-workers exploited the advantage of the phase-changing property of gelatin, a highly biocompatible material, as bioink for creation of perfusable vascular channels. ${ }^{[210]}$ Exactly like Pluronic F127 but reversed in phase, the gelatin solution (>10 w/v.\%) forms a hydrogel at room temperature and changes back to the solution phase at $37^{\circ} \mathrm{C}$, which enabled spontaneous dissolution of the liquefied bioink over the subsequent cell culture process.

Mechanical extraction of the bioprinted templating microfibers from fabricated tissue blocks has also been demonstrated as an efficient strategy to create vascular structures. In this case, 
Khademhosseini and co-workers adopted a phase-changing agarose solution as the sacrificial template (Figure 15C). ${ }^{[209]}$ Agarose solution is in liquid form at temperatures of $>80{ }^{\circ} \mathrm{C}$ and solidifies with a strong mechanical strength when cooled down to room temperature. This allowed it to be easily extruded and then extracted manually or using a mild vacuum from a hydrogel matrix, leaving hollow channels that could subsequently be endothelialized. Hydrogel blocks containing different forms of interconnected vascular network were fabricated based on this versatile approach (Figure 15D). Endothelialization is typically achieved by infusing high concentrations of endothelial cells into the created microchannel networks. By properly controlling the conditions for the seeding process, the endothelial cells could form well-stretched monolayers in the inner surface of the microchannels mimicking the intact endothelium, where expression of functional biomarkers could be observed.

Moreover, Khademhosseini, Annabi, and co-workers achieved the bioprinting of bone-like tissue constructs involving vascular structures, approaching the problem of proper perfusion for larger-scale tissue constructs, by using a central vascular structure in the middle of the construct. ${ }^{[124]}$ Their method was based on direct-write bioprinting of different GelMA-based bioinks to achieve a gradient-like structure (Figure 16A). Hereby different cylinders of extruded bioink were bioprinted in a controlled manner to achieve a defined bone-like structure. GelMA at a high degree of methacryloyl substitution embedded with silicate nanoparticles was used to achieve osteogenic differentiation of loaded MSCs into the bioink and bioprinted around a central cylinder, which was made of GelMA at a very low percentage of methacryloyl substitution loaded with HUVECs to achieve the formation of perfusable blood vessels. In addition, the GelMA bioink serving as central column was modified to conjugate VEGF to the polymer backbone to achieve increased vascular formation in the center of the construct. After a culture period of 7 days, the central column was fully degraded (rather than removal immediately post-bioprinting in the case of sacrificial bioprinting), whereas the surrounding construct remained unaffected leaving a perfusable blood vessel-like structure (Figure 16B). They sustained cell viability and activity over a duration of 21 days, as well as induced osteogenic differentiation of the bioprinted MSCs and the formation of perfusable blood vessels which was verified by immunohistochemical staining of Alizarin Red S and alpha smooth muscle actin (aSMA) for the respective tissues (Figure 16C and 16D). Through the formation of perfusable blood vessels in a biomimetic gradient bone tissue, they proved the capability of their 3Dbioprinted tissue to mimic structural gradients as well as the vascularization of human tissues, which is crucial for the functionality of bioprinted construct in a realistic in vivo environment.

Although the sacrificial bioprinting provides a versatile method to create vessel-like structures, the patterns that it can achieve are still relatively simple. The fabrication of complex cell-laden vascular structures remains challenging because of the limitation in the attainable scale of constructs and the time needed to remove the sacrificial layer after bioprinting.

To this end, Chen and co-workers developed an approach to directly bioprint more complex vasculature in tissue-like matrices such as pre-vascularized liver tissues, using a novel 
stereolithography-based bioprinting approach termed the microscale continuous optical bioprinting ( $\mu \mathrm{COB}$ ) (Figure 16E). ${ }^{[213]}$ They included several native vascular cells such as HUVECs and C3H/10T1/2s into a GelMA-HA hydrogel composition, which they then bioprinted into a vascular structure by photocrosslinking the representative areas required to form a vascularized structure. In between the vascular structures, they bioprinted a GelMA hydrogel encapsulating HepG2 cells. Their bioprinted tissues could form lumen-like vascular structures in different complexities and gradients in vitro without the necessity of a sacrificial layer (Figure 16F). CD31 staining revealed that that the bioprinted HUVEC formed conjunctive networks, whereas staining for aSMA indicated that the bioprinted 10T1/2 cells displayed a pericyte-like phenotype. Subcutaneous implementation of their constructs into severe combined immunodeficiency (SCID) mice showed proper viability and performance of the bioprinted construct in vivo, which was confirmed by harvesting and staining of the grafted tissues 2 weeks after implementation. Furthermore, by intravenous injection of mouse- and human-specific lectin, that exclusively binds to the respective endothelial cells, they observed anastomosis between the bioprinted network and the host circulation resulting in functional blood vessels. As they were able to prove the performance of their constructs in an in vivo environment, this method of bioprinting pre-vascularized structures might be a step towards clinical translation of bioprinting.

The current research on proper vascularization of bioprinted constructs is a crucial step towards clinically relevant 3D-bioprinted tissues. Especially combining vascular structures with gradients, for example to mimic bone tissue, or taking the tissue-specific geometry, such as hexagonal structures in the liver, into account shows great potential for the successful fabrication of biomimetic organs and tissues.

Alternatively, standalone vascular structures can be bioprinted by for example utilizing the rapid $\mathrm{Ca}^{2+}$ crosslinking of sodium alginate. ${ }^{[214,215]}$ By applying this strategy, Ozbolat and co-workers were able to fabricate vascular structures by use of a coaxial nozzle. Here, the outer channel of the coaxial nozzle was stationed with sodium alginate, while the inner channel was flowed with $\mathrm{CaCl}_{2}$. When $\mathrm{Ca}^{2+}$ and sodium alginate converged at the tip of nozzle, hollow physically crosslinked alginate filaments were formed, assisted by the continuous flowing of the two phases. Similarly, $\mathrm{He}$ and co-workers used this technique combined with an X-Y-Z robotic stage to generate 3D tissue constructs with built-in perfusable microchannels (Figure 17A). ${ }^{[214]}$ It was also proven that the viability of L929 mouse fibroblasts was as high as 92\% after 1 day of incubation (Figure 17B). Zhang, Shin, and Khademhosseini and co-workers further reported an approach to directly write perfusable vascular structures by the application of a multi-layered co-axial extruder (Figure 17C). ${ }^{[216]}$ By using a blend bioink consisting of GelMA, sodium alginate, and 4-arm PEGtetraacrylate (PEGTA), they were able to produce well-arranged vascular structures within a single-step bioprinting process. During bioprinting the bioink was ionically crosslinked with $\mathrm{Ca}^{2+}$ for the alginate component of the bioink, followed by exposure to UV light resulting in a chemical crosslinking of the GelMA and PEGTA components post-bioprinting. High cell viability and spreading of the encapsulated endothelial cells and MSCs could be obtained resulting in highly organized, biologically relevant, and perfusable vessels (Figure 17D and 17E). More recently, the same group around Zhang, Khademhosseini, and co-workers fabricated multi-layered cannular tissues using a multi-channel coaxial extrusion system. 
[182] They demonstrated bioprinting of single- or multi-layered hollow fibers with complex cellular composition as found in tubular tissues such as blood vessels, trachea, ureter, or urethra. In particular, the encapsulation of human urothelial and bladder SMCs on the one hand resulted in the fabrication of functional cannular urothelial tissue constructs. The encapsulation of HUVECs or human SMCs on the other hand facilitated the bioprinting of dual-layered blood vessels.

Another promising approach to achieve vascular structures is the scaffold-free bioprinting of blood vessels. Forgacs and co-workers described a method for scaffold-free fabrication of small-diameter blood vessels using an extrusion-based bioprinting platform. ${ }^{[217]}$ Spheroid or cylindrical-shaped aggregates $(300-500 \mu \mathrm{m})$ of SMCs and fibroblasts were extruded surrounded by agarose rods serving as a molding template. After bioprinting, the spheroids or rods could fuse resulting in single- or double-layered vessels with an outer diameter ranging from 0.9 to $2.5 \mathrm{~mm}$ (Figure 17F). A similar approach was adopted by Koc and coworkers, who were able to bioprint spheroids and cylindrical cell aggregates consisting of immortalized mouse embryonic fibroblasts (MEFs) into circular shapes of sizes ranging from 4 to $10 \mathrm{~mm}$ as a surrounding template. ${ }^{[218]}$ Similar to the method by Forgacs and coworkers the spheroids eventually fused to form a blood vessel.

In addition, a "Kenzan" method was developed for nearly scaffold-free bioprinting. Cellular spheroids were skewed onto an array of needles controlled by a computer system, which allowed to import different CAD models (Figure 17G and 17H). Morita and co-workers used this technique to generate tubular tissues out of spheroids including 40\% HUVECs, $10 \%$ smooth aortic muscle cells, and 50\% human dermal fibroblasts. ${ }^{[219]}$ They successfully fabricated a 7-mm long vessel with $1.5 \mathrm{~mm}$ of diameter. Final in vivo implantation into rats exhibited remodeling and endothelialization of the tubular construct after 5 days. This method can be of particular interest in the fabrication of 3D constructs as it fully avoids the need of a bioink as support. Recently, Moldovan and co-workers summarized the advantages of the "Kenzan" method in their review, stating the advantages in the fabrication of biologically relevant blood vessels. ${ }^{[220]}$

\subsection{Neuronal tissues}

Besides mimicking the multiple components of biomimetic tissues and proper nutrition and oxygenation delivery via vascular structures, the integration of structures of the nervous system is another crucial step towards the clinical translation of bioprinted constructs. The main function of the nervous system can be summarized as transmitting signals to and from parts of the body, ultimately controlling its actions. In vertebrates the nervous system can be generally divided into the central nervous system (CNS) and the peripheral nervous system (PNS). ${ }^{[141]}$

The CNS consists of the brain and the spinal cord, which is the integrating and command center of the nervous system. ${ }^{[141]}$ Bioprinting research on the CNS is primarily focused on the understanding of the brain and treatment of neurodegenerative diseases such as Alzheimer's or nerve injuries. ${ }^{[221,222]}$ Zhang and co-workers used electrospinning combined with stereolithography for the fabrication of a 3D biomimetic neural scaffold (Figure 18A - 18C). ${ }^{[223]}$ By combining PCL with gelatin, they created a bioink that showed 
enhanced neural cell adhesion, survival, and proliferation. Confocal microscopy revealed that their PCL/gelatin fibers greatly increased the length of neurites and directed neurite extension of primary cortical neurons along the fiber. Recently, Wallace and co-workers presented a new approach to better mimic the complex 3D structure of the human brain by including primary neural cell-loaded hydrogels in combination with extrusion-based 3D bioprinting. ${ }^{[221]}$ In brief, they developed a new peptide-modified polysaccharide, gellan gum-RGD (RGD-GG), and combined it with primary cortical neurons. Their results indicated enhanced proliferation and network formation of the cortical cells in a layered 3D construct (Figure 18D and 18E). The model demonstrated superior performance to comparable 2D models of the human brain and formed an enabling platform to understand brain damage or neurodegenerative diseases.

The PNS on the other hand, comprises all the nerves that are not part of the CNS. It mainly consists of spinal nerves, carrying impulses to and from the spinal cord, and cranial nerves, carrying impulses to and from the brain. ${ }^{[141]}$ The PNS forms the main communication line between the different parts of the body and the CNS. ${ }^{[141,224]}$ Peripheral nerve injury is a disease with significant influence on a patient's quality of life as well as high impact on health care. ${ }^{[225]}$ Haycock, Boissonade, and co-workers developed nerve guidance conduits (NGCs) as alternatives to common autografts as treatment for nerve injury. ${ }^{[222]}$ They bioprinted the NGCs incorporating Schwann cells and dorsal root ganglions in a PEG resin using laser-based microstereolithography ( $\mu$ SL, Figure 18F). Their method might be an important step towards a better model of nerve injury as well as an alternative treatment for larger-scale nerve damage. Zhang and co-workers approached the medical need to repair nerve damage by developing NSC-laden GelMA/bioactive graphene platelet 3D constructs, bioprinted using stereolithography. ${ }^{[226]}$ Their 3D-bioprinted constructs could promote cell survival and growth in a well-defined bioactive architecture. Furthermore, they proved NSC differentiation and neurite elongation after 2 weeks of culture. Recently, Ning and coworkers developed a scaffold containing alginate, fibrin, HA and RGD peptide for the applications in nerve tissue engineering. ${ }^{[227]}$ Incorporated Schwann cells into the construct displayed high cell viability of $95 \%$ after 10 days of bioprinting as well as alignment of cells among the scaffolds promoting the extension of dorsal root ganglion neurites. More recently, a similar approach was taken by Zhou, Xu, and co-workers, who encapsulated rat Schwann cells into a gelatin-alginate composite bioink. The 3D bioprinted cultures displayed higher nerve growth factor release compared to conventional 2D monolayer culture as well as high expression of the Schwann cell-specific marker S100 $\beta$, proving the advantage of 3D nerve cultures.

The recent trends in bioprinting have allowed for the fabrication of direct neural structures that mimic the function of neural tissue in a realistic environment. Although these 3Dbioprinted neural tissues are still in infancy and not yet applicable for in vivo environments, the recent demonstrations form a promising basis for further research in the field of biomimetic neural tissue bioprinting. However, more research and in particular in vivo evaluation of bioprinted neural structures are needed before these can be translated into the clinics. 


\subsection{Cartilage and bone tissues}

Another field of primary interest is the regeneration of cartilage tissue. Cartilage is known to regenerate very slowly but the number of injuries or diseases (especially osteoarthritisrelated) resulting in cartilage damage are increasing annually. ${ }^{[228]}$ As a result, novel strategies are needed for successful treatment and replacement of cartilage tissues. To this end, 3D bioprinting offers a promising platform for the rapid production of cartilage tissues aiming to replace diseased counterparts. Gatenholm and co-workers presented an extrusionbased bioprinting platform to generate larger-scale cartilage tissues such as a human ear or a sheep meniscus using nano-fibrillated cellulose with alginate. ${ }^{[229]}$ Their designs were based on original MRI scans as templates, demonstrating the capacity of 3D bioprinting as a tool to create tissues on demand. Studies with human chondrocytes have shown acceptable viability in these scaffolds of approximately $70 \%$ directly after bioprinting and $85 \%$ after 7 days of culture, presenting the high potential of this method and the used materials to serve as suitable tool for the fabrication of tissues for cartilage replacement. This research, however, displayed one of the major drawbacks of current bioprinting for rigid tissues such as cartilage or bone - the necessity of a rigid scaffolding material such as PCL. As previously mentioned in Section 3.1, most common hydrogels such as alginate and GelMA are too soft to be used in cartilage or bone tissue engineering. Therefore, most approached typically rely on scaffold-based designs or blends with rigid hydrogels to successfully bioprint cartilage- or bone-like tissues. Cho and co-workers described a PCL-alginate-based scaffold enriched with transforming growth factor- $\beta$ (TGF $\beta$ ) incorporating chondrocytes. [230] Implantation of these constructs into the subcutaneous space of nude mice displayed enhanced cartilage tissue and type II collagen fibril formation after 4 weeks of experimentation, which was analyzed by Alcian blue, hematoxylin \& eosin (HE), and collagen type II staining.

The scaffold approach can also be used for bioprinting tissues aimed for bone regeneration. Zhang and co-workers designed 3D-bioprinted micro- and nano-scaffolds for vascularized bone tissue repair. ${ }^{[231]}$ In their work they described the fabrication of combined micro- and nanoscale scaffolds to induce bone formation including highly interconnected 3D microvascular-mimicking channels. The scaffolds were based on polylactic acid (PLA), later modified with nano-HA conjugation, enhancing the osteogenic differentiation. Their results proved enhanced bone-like physical properties as well as vascular flow profiles. Cell studies including MSCs exhibited enhanced cell adhesion, proliferation, and osteogenic differentiation, as well as improved vascular cell growth, migration, and organization with HUVECs.

Atala and co-workers further modified the scaffold approach by using a combination of cellladen hydrogel fibers, consisting of gelatin, fibrinogen, HA and glycerol, as well as mechanically supporting biodegradable PCL fibers anchored on a sacrificial Pluronic F-127 hydrogel construct (Figure 19A). ${ }^{[232]}$ Based on this technique, they developed an integrated tissue-organ bioprinter (ITOP), which was able to bioprint complex human tissues with correct shapes for the reconstruction of mandible and calvarial bone and ear cartilage based on MRI and computer tomography (CT) scans (Figure 19B - 19H). By the integration of microchannels they secured the diffusion of nutrients to the cells, which allowed bioprinting 
of larger constructs. All constructs demonstrated good cell behavior and tissue-like properties, as well as long-term stability after 5 months of implantation into the calvaria or skullcap (Figure 19I). Their newly developed bioprinting platform is a promising tool to bioprint on-demand tissues for regenerative medicine.

More recently, Oliveira and co-workers demonstrated the possibility of bioprinting for the in-situ treatment of patient wounds by fabricating bone substitutes using laser-assisted bioprinting. ${ }^{[233]}$ They were able to bioprint cell discs and rings containing MSCs in combination with a collagen/hydroxyapatite bioink focusing on proper alignment of cells to represent the bone architecture. In vitro evaluation showed osteoblastic differentiation and bone tissue formation as well as high metabolic activity of cells. Implantation of the bioprinted bone discs into Balb/c mice with induced defect of the circular bone revealed enhanced regeneration of the bone tissue and high survival of implanted cells 42 days after surgery.

In summary, the approaches mentioned above have shown the promising application of 3D bioprinting in the treatment of cartilage defects or damages in the bone tissue. Especially due to the use of MRI and CT scans as digital templates, 3D bioprinting can enable the generation of the complex human architecture of representative tissues with biomimetic properties and physiology. In vivo implementation of the bioprinted tissues has demonstrated the performance of 3D bioprinting in tissue engineering for bone and cartilage repair.

\subsection{Cardiac tissue}

The bioprinting of a functional myocardium remains as a key challenge in tissue engineering and regenerative medicine, as it has been difficult to achieve functional heart-like tissues with well-defined alignment of cells combined with the capability to contract. ${ }^{[141]}$ Zhang, Khademhosseini, and co-workers developed a method to use 3D bioprinting for the fabrication of endothelialized myocardium. ${ }^{[234]}$ They used an endothelial cell-laden hybrid bioink containing sodium alginate and GelMA to bioprint a scaffold for later seeding with cardiomyocytes (Figure 20A). Primary crosslinking of alginate during the bioprinting process with $\mathrm{Ca}^{2+}$ followed by subsequent UV crosslinking of the GelMA component allowed for the fabrication of a rigid 3D structure. The laden endothelial cells migrated towards the peripheries of the bioprinted fibers and formed a confluent layer of endothelium. Seeding of cardiomyocytes into this scaffold combined with controlled anisotropy resulted in an aligned myocardium that could contract in a spontaneous and synchronous manner proven by immunofluorescent staining of sarcomeric a-actinin and connexin-43 ( $\mathrm{Cx} 43)$ as well as measurements of the contraction amplitudes (Figure 20B - 20D). By combining these two cell types they bioprinted a biologically relevant myocardium that demonstrated the typical contraction behavior of the human counterpart as well as the formation of an interlacing endothelium. Although this bioprinted endothelialized myocardium was designed for the use in a heart-on-a-chip platform and was not yet a suitable transplantable biomimetic tissue, such an approach to bioprint a vascularized myocardial tissue is a step towards the generation of functional cardiac tissues with biomimetic architecture and function. 
Guo and co-workers developed a conductive bioink based on conductive nanofiber yarns encapsulated into GelMA hydrogel shells (Figure 20E). ${ }^{[235]}$ The conductive nanofiber yarn networks (NFYs-NETs) were fabricated using EBB of PCL, silk fibroin (SF), and CNTs. They proved that their NFYs-NETs were able to control cellular orientation and enhance cell maturation of seeded cardiomyocytes (Figure 20F). Embedding these NFYs-NETs into GelMA hydrogel shells further promoted cell alignment and maturation. In addition, they seeded endothelial cells in the GelMA hydrogel shells to better mimic the structure of an endothelialized myocardial tissue. They demonstrated cell viability for a total duration of 8 days and enhanced alignment and maturation of cells in comparison to conventional 2D cell cultures (Figure 20G). Although these 3D-bioprinted constructs have not yet been tested in vivo, they hold great potential for the fabrication of biologically relevant biomimetic cardiac tissues. Moreover, Hibino and co-workers applied the previously described "Kenzan" technique to bioprint cardiac patches consisting of iPSC-derived cardiomyocytes, human fibroblasts, and endothelial cells. ${ }^{[236]}$ They observed spontaneous beating of the patches after in vitro culture of 3 days combined with ventricular-like action potentials and electrical conduction throughout the whole construct. Furthermore, they confirmed the presence of $\mathrm{Cx} 43$ at the cell boundaries, proving the development of a functional cardiac tissue. In vivo implementation of the patch into nude rats resulted in vascularization of the cardiac patches with observed engraftment into the native rat myocardium. As the authors were able to demonstrate the performance of their patch in vivo, it might indicate the potential of clinical translation of these 3D-bioprinted cardiac tissues. Similarly, Bearzi, Rizzi, and co-workers recently bioprinted cardiac tissues composed of HUVECs and iPSC derived cardiomyocytes based on an alginate/PEG-fibrinogen bioink. ${ }^{[237]}$ Their 3D cardiac tissues showed high cell alignment as well as the formation of blood vessels, which upon in vivo implementation into non-obese diabetic SCID mice showed high integration with the host vasculature, proving the functionality of their bioprinted cardiac tissues. In particular, the combination of their bioprinted construct with patient-derived iPSCs might be a significant step towards the personalized treatment of patients and might have significant impact on regenerative medicine.

The recent advances in the fabrication of cardiac tissues have demonstrated the applicability of bioprinting for the treatment of severe medical conditions such as heart failure. Especially the demonstration of the cardiac-tissue specific alignment and beating of the cells in vitro and in vivo, shows that bioprinting is not only able to mimic structural characteristics of biological tissues but is also capable of exhibiting tissue-specific behavior and functionality.

\subsection{Skeletal muscle}

Besides the research on 3D-bioprinted cardiac tissues, several studies have also focused on the generation of functional skeletal muscle tissues. Bioprinting of muscle tissue is a peculiar challenge as bioprinted structures should show a well-defined alignment of included cells combined with the capability to contract, based on electric stimuli. ${ }^{[141]}$ Recently, Rainer and Gargioli, and co-workers described a method for 3D bioprinting of skeletal muscle tissues with functional morphology and properties. ${ }^{[238]}$ They combined a photocurable semi-synthetic biopolymer made of PEG-fibrinogen encapsulating $\mathrm{C} 2 \mathrm{C} 12$ cells (Figure 21A). By extruding the cell-laden bioink in an aligned manner to form hydrogel 
fibers, migration and fusion of cells were observed after 3-5 days of culture resulting in aligned myotubes, which was confirmed by immunofluorescent staining against myosin heavy chain (MHC). They showed a high degree of alignment of laden cells in fiber direction, maturation, functionality, as well as increased sarcomerogenesis (Figure 21B). By implantation into SCID mice, they proved the generation of organized muscle tissues in vivo after a period of 28 days by retrieving, sectioning and staining the representative tissue grafts (Figure 21C and 21D). Their approach displayed the functionality of 3D-bioprinted muscle tissues for in vivo applications and formed an important step towards the production of biomimetic muscle tissues for medical applications. Atala and co-workers also reported the fabrication and evaluation of a 3D-bioprinted skeletal muscle tissue in their work (Figure 21E). ${ }^{[232]}$ By using their newly developed ITOP platform, 3D muscle constructs encapsulating mouse myofibroblasts of $15 \times 5 \times 1 \mathrm{~mm}^{3}$ in size were bioprinted (Figure $21 \mathrm{~F})$. In vitro experiments suggested cell maturation and alignment along the bioprinted fibers, indicating the suitability of the bioprinted constructs for regeneration purposes. At 2 weeks after implantation, the muscle constructs were analyzed and shown to exhibit proper cell alignment and clusters of acetylcholine receptor (AChR) in the muscles fibers, as well as contact with neurofilaments showing nerve integration into the model (Figure $21 \mathrm{G}$ - 21I). Recently, Lee and co-workers used the same ITOP bioprinting platform to fabricate a 3D implantable muscle constructs incorporating primary human muscle progenitor cells. ${ }^{[239]}$ Their bioprinted muscle constructs displayed a highly packed and organized structure of viable and aligned myofiber-like structures. In vivo studies using a rodent model of a tibialis anterior muscle defect showed $82 \%$ muscle function recovery after 8 weeks of implantation, demonstrating how bioprinted muscle constructs can be used to treat severe muscle defects.

In summary, the recent developments in 3D-bioprinted muscle tissues have demonstrated the high potential of these constructs for application in regenerative medicine. In particular, successful implantation of 3D-bioprinted constructs in in vivo models proved the performance and functionality of these biomimetic tissues. Furthermore, the recent progress in this field displays the capability of 3D bioprinting to fabricate tissues that are capable of responding to electronic stimuli, which can be found in human muscle tissues. The described research is a crucial step for the fabrication of biomimetic tissues.

\subsection{Summary on the current status of bioprinted tissues}

As discussed above, several approaches have emerged over the last years with the aim to bioprint biomimetic tissues for organ translation. The current trend shows that common challenges, like the proper nutrition of larger-scale samples or the appropriate alignment of muscle fibers, are addressed by developing novel bioprinting strategies. These especially focus on incorporating vascular structures into the bioprinted constructs or mimicking the natural architecture and functionality of a tissue. The medical need for larger-scale biomimetic tissue constructs and transplants has resulted in novel constructs that are able to mimic the function of cartilage, bone, and muscle tissues, respectively. These bioprinted structures are not only presenting great and promising results in vitro but also succeed after implementation into animal models proving that 3D bioprinting is able to produce suitable organ mimics that have translational potential. The trend to combine proper nutrition and oxygenation via blood vessels, gradient structures within a single construct and a bioink 
design that increases cell viability, maturation, and differentiation will eventually lead to bioprinted constructs that are well mimicking realistic human tissues. In such way bioprinted tissues might be able to support or replace damaged tissues like bone, cartilage, muscle and even neural tissue. Nevertheless, so far only a few studies have successfully evaluated the performance of bioprinted constructs in an in vivo environment. More studies need to focus on the implantation of constructs into a realistic environment (e.g., into mice, rats, etc.) to demonstrate the possible translation of research to the clinics. However, full clinical translation of bioprinted constructs still has a long way to go as robust and systemic evaluations are necessary to prove in vivo applicability of bioprinted constructs. Although numerous studies mentioned in this review haven indicated the biocompatibility of their constructs, their bioactivity and functionality, as well as parameters such as degradation profiles and possible immune responses of the bioinks utilized, need to be characterized in a case-specific manner in future clinical trials. Nevertheless, with the growing tendency towards in vivo models and the promising results obtained from the aforementioned studies, it is reasonable to anticipated that $3 \mathrm{D}$ bioprinting will achieve clinical acceptance in the near future.

\section{Commercially Available Bioprinters}

The many opportunities of bioprinting for tissue engineering and regenerative medicine has led to the foundation of many companies that start producing commercially available bioprinters (Figure 22). Here we introduce a few representative companies based on different bioprinting technologies, which have either introduced a novel technique to the market or presented an innovative approach to improve the efficiency or affordability of current techniques.

Organovo, a company based in San Diego, CA, USA, was the first company to commercialize 3D bioprinting. ${ }^{[240]}$ Their bioprinter "NovoGen" is a platform using pistonbased extrusion for material deposition. From this starting point, several different companies were subsequently founded, which has made 3D bioprinting widely available for commercial use. The products range from large-sized bioprinters only available for research to desktop bioprinters that can fit in most laminar flow hoods. Remarkable for all systems is that they have mainly chosen syringe-based extrusion as the extrusion strategy. This allows the diameter of the extruded bioink fiber to be individually adjustable depending on the gauge size of the needle used. It also allows for a simple replacement of different bioinks by replacing the syringe.

Over the last decade, several other 3D bioprinters have been developed ranging from affordable bioprinters such as the Allevi 1/2, a dual extrusion bioprinting system from Allevi (Philadelphia, PA, USA) ${ }^{[241,242]}$ or the Inkredible from CELLINK (Gothenburg, Sweden) $[242,243]$ to the BioAssemblyBot form Advanced Solutions (Louisville, KY, USA), a highcost syringe-based extrusion bioprinter consisting of a six-axis robotic arm. ${ }^{[244]}$

As abovementioned, stereolithography forms a promising platform for tissue engineering and regenerative medicine applications. However, no stereolithography-based bioprinters are so far commercially available yet. Nevertheless, printers such as the Formlabs desktop 
stereolithography printer Form 2 (Formlabs, Somerville, MA, USA), the Ember Stereolithography 3D Printer (Autodesk, San Rafael, CA, USA), or the LittleRP Open 3D Resin Printer (Little RP, Santa Barbara, CA, USA) have high potential to serve also as bioprinting platforms. ${ }^{[245]}$ Although these are not officially available as bioprinters, the stereolithography technique might still be applicable for hydrogels instead of the commercially available resin with proper optimizations of the printing parameters. This assumption remains to be proven experimentally.

Cyfuse Biomedical (Tokyo, Japan) offers a new bioprinting method using the "Kenzan" technique. ${ }^{[246]}$ Cell aggregates are sewed onto an array of fine needles precisely controlled by a computer program. Over time the cell aggregates fuse to form a uniform cell layer, which then can be cultured and/or perfused with medium. Aspect Biosystems (Vancouver, Canada) has developed a bioprinter that combines a microfluidic printhead, capable of extruding multiple materials through the common core while the crosslinking agent $\mathrm{CaCl}_{2}$ solution through the sheath, onto a moveable stage. ${ }^{[247]}$ The microfluidic chip allows rapid exchange of materials during the bioprinting process, resulting in generation of heterogeneous tissues.

Another approach of a commercially available bioprinting system for spheroid-based tissue biofabrication is offered by n3D Biosciences, Inc. (Houston, TX, USA). ${ }^{[248]}$ Their bioprinting kits featuring extremely low costs allow for the assembly of cellular spheroids of different diameters depending on the chosen product type. They use magnetic levitation as the method to form spheroids within minutes or hours. n3D Biosciences has made it possible to fabricate 3D structures without the necessity of purchasing a conventionally defined 3D bioprinter. However, the constructs are limited to smaller-sized spheroids (ranging from 1 to $3 \mathrm{~mm}$ ) and the platform is not directly suitable for the production of larger-scale tissue constructs.

The recent developments in commercially available bioprinting systems form a crucial part in the field of bioprinting as it allows scientists from different expertise who do not necessarily have knowledge of operating a bioprinter, to participate in the research on 3D bioprinting, eventually leading to improvements on technologies for generating functional tissue constructs targeted towards biological studies and translational applications.

\section{Future Directions \& Challenges}

Over the past years, the field of 3D bioprinting has rapidly progressed from proof-of-concept prints to complex multi-component tissue-like constructs that are similar to their in vivo counterparts. Nevertheless, the full clinical translation of bioprinted tissues is exceedingly challenging and it will take a long time until 3D-bioprinted tissues fully manifest themselves for the transplantation of organs or the reconstruction of damaged tissues such as nerves or cartilage. Although the progress of bioprinting in the recent years is impressive, there are still certain limitations that need to be overcome, which will be discussed in greater detail in this section. 
First, bioprinting systems have become progressively complex over the last years resulting in the fabrication of 3D volumetric constructs with highly controlled architecture and composition. Recent trends towards systems that are capable of integrating multiple materials into a single construct with different mechanical properties or cellular composition, fabricating hollow perfusable vessel-like constructs embedded in bioprinted tissues or depositing structures that change their conformation based on external stimuli facilitate the fabrication of biologically relevant tissues that might find application in the clinics in near future. However, a common disadvantage of current bioprinting systems is the required time to fabricate complex biomimetic tissues based on low bioprinting speed that can be achieved. This is a significant limitation towards clinical application of bioprinting as larger bioprinted tissues might exceed the available time to create tissues on demand. Above all, switching between different materials during the bioprinting process or bioprinting in very high resolution can significantly increase the time necessary to fabricate a functional construct. Although some studies focus on decreasing the required time by for example using a single nozzle to deposit multiple materials in extrusion-based bioprinting, ${ }^{[174]}$ the overall speed that can be achieved remains insufficient to create on-demand tissues. Stereolithography might offer a bioprinting platform that can achieve higher bioprinting speeds due to the large surface area that can be simultaneously crosslinked in the process. However, up to now stereolithography cannot reach the versatility of extrusion-based bioprinting and only a few studies successfully integrated multiple materials in a stereolithography-based system. ${ }^{[180]}$ The improvement of bioprinting speeds is an essential step towards clinical translation of bioprinting and the on-demand fabrication of biologically relevant tissue constructs.

Another aspect of bioprinting that has been rapidly evolved over the last years is the research on novel bioinks that allowing replicating organ-specific ECM while maintaining high cell viability and activity as well as induce organ-specific cell behaviors. Several different compounds have been extensively investigated for their application to fabricate biomimetic tissues and novel compounds are continuously developed to achieve improved biological resemblance of bioprinted constructs. However, often the bioink remains a "synthetic" environment that might induce organ-like behavior of cells but only hardly mimics the complete actual composition of the organ-specific ECM. To this end, dECM offers a promising solution to this problem as $\mathrm{dECM}$ can be directly obtained from the desired tissue including all compounds and growth factors that are characteristic for the natural tissue. However, dECM in bioprinting applications is often based on donor organs from animals, which might not fully replicate the natural ECM that can be found in human tissues. Moreover, the process to retrieve, purify and modify the matrix is time-consuming and the amount of bioink that can be obtained is limited. ${ }^{[249]}$ Nevertheless, dECM remains one of the most promising sources to achieve bioink that are capable of fully mimic the complex environment composition of realistic tissues and more research might result in wider application of dECM in bioprinting. An approach towards more personalized bioinks was recently investigated by Tamayol, Ruskin, and co-workers. ${ }^{[250]}$ They developed a bioink including autologous patient material obtained from platelet-rich plasma to achieve patientspecific bioprinted constructs. Their approach demonstrates that patient-specific tissues can be obtained by bioprinting and especially in the clinics the use of autologous materials is a 
crucial factor to ensure tissue performance and transplantability. dECM enriched with autologous growth factors obtained from patients might form the future for patient-specific bioinks that are suitable for the application in clinics and for the transplantation of bioprinted constructs into the human body.

A general challenge of bioprinting is mimicking the complexity of desired organ structures. Although recent studies try to mimic the detailed architecture and composition of natural tissues and even incorporate 4D bioprinting strategies as discussed in greater detail in Section 5.5, it is still not possible to fully mimic the complexity and functionality of natural organs. One of the major drawbacks of current bioprinting systems is the low resolution compared to natural tissues. Most structures of organs and tissues are finer and more delicate than current bioprinters can achieve. Although EBB might be capable of fabricating constructs with very high resolution, it is until now not possible to encapsulate cells in the process, making it unsuitable for most bioprinting applications. Bioprinting strategies need to be improved to achieve a resolution that is relevant for natural tissues. Furthermore, currently bioprinted tissues are mostly limited to 3-4 different types of cells within a single construct as described above. However, natural tissues often consist of more cell types that require constant crosstalk with surrounding cells to proliferate and display their specific function in vivo. ${ }^{[251]}$ Replicating this particular crosstalk and interaction in vitro is exceptionally challenging due to the different tissue-specific requirements of all cells. For example, astrocytes, microglia, oligodendrocytes and neurons in the brain all require different media including specific growth supplies to fully proliferate and function in vitro. To co-culture multiple cells in a single bioprinted tissue, one must optimize the culture conditions respective to the used cells and the targeted tissue matching all necessary requirements for individual cells. More fundamental research is required that in particular, focuses on improving the current culture conditions to enable proper maturation of multicellular 3D bioprinted tissues.

Despite several challenges that remain to be solved to achieve full clinical translation of bioprinting, there are also highly promising advancements that have emerged over the last years. A positive development is the focus on proper vascularization of tissues and the use of multiple materials within a single construct that allows for the design of gradients similar to the structures of natural tissues. The number of materials that can be bioprinted in a single bioprinting system continuously grows as discussed above. Furthermore, a promising tendency of most recent studies is the evaluation of bioprinted tissues in vivo. Demonstrating the function of constructs in a realistic environment is a crucial step towards the clinical translation of bioprinting. Recent progress shows highly auspicious results and could proof the performance and stability of bioprinted bone, cartilage and muscle tissues in biological conditions. In vivo experiments should be an essential part for all bioprinting studies that focus on the fabrication of biomimetic tissues suitable for possible translation into the clinics.

At last, the continuously growing number of bioprinting companies makes bioprinting more accessible to the public, which eventually will lead to more research on bioprinting and ensures further developments and achievements. Nevertheless, most available bioprinting platforms are based on extrusion-based bioprinting and only a few try to implement different 
techniques into their system. So far, no commercial bioprinters are available that are based on stereolithography. Although a couple conventional 3D stereolithography printers show the potential to be modified into fully functional bioprinters in the near future. To extend the possibilities of bioprinting and the future application of bioprinting in the clinics, it is necessary that more companies focus on the use of different techniques in their systems to overcome current limitations as discussed above. Nonetheless, the tendency that more and more bioprinting systems are accessible and affordable to the public will have a positive effect on the research in this field.

In summary, the recent progress in bioprinting is impressive and it is almost certain that the field will continue to grow and evolve in the next years. Nevertheless, a lot of challenges remain that limit the current performance of bioprinting. Mimicking the complexity of organs is one of the major difficulties bioprinting is currently facing. Reconstructing the delicate architecture, complex cell and material composition as well as the tissue-specific functionality remains unachievable with current bioprinting techniques. Furthermore, standard cell culture conditions and techniques might not fit the requirements for the culturing and maturation of 3D-bioprinted tissues. More research focusing on the fundamental techniques might help to achieve complex biomimetic constructs.

\section{Conclusions}

Three-dimensional bioprinting is a field that has been rapidly growing over the last decade and will continue to grow until it is an established method in the clinics. The production of fully biomimetic transplants is the major focus of several studies and current results promise a bright future for the field of bioprinting. Although most of current bioprinted tissue constructs are still infant and only mimic relevant biological tissues, trends to focus on proper vascularization of the tissue, multi-cellular designs and high-resolution bioprinting form the foundation of producing functional tissues that are suitable for clinical translation. The recent trend towards evaluation in an in vivo environment has demonstrated that the bioprinted constructs are able to perform their functions to varying degrees. It is anticipated that $3 \mathrm{D}$ bioprinting will continue to evolve and will find its way from the benches of scientific research towards translational applications in regenerative medicine.

\section{Acknowledgments}

The authors gratefully acknowledge funding from the National Institutes of Health (K99CA201603, R21EB025270, R21EB026175).

\section{References}

[1]. a) Langer R, Vacanti J, Science 1993, 260, 920; [PubMed: 8493529] b) Khademhosseini A, Vacanti J, Langer R, Sci. Am. 2009, 300, 64.

[2]. Zhang Y, Xia Y, Nanomedicine 2015, 10, 689. [PubMed: 25816873]

[3]. Place E, Evans N, Stevens M, Nat. Mater. 2009, 8, 457. [PubMed: 19458646]

[4]. Atala A, Kasper F, Mikos A, Sci. Transl. Med. 2012, 4, 160.

[5]. Priya S, Jungvid H, Kumar A, Tissue Eng. Part B: Rev. 2008, 14, 105. [PubMed: 18454637]

[6]. Saladin K, Miller L, Anatomy \& physiology, McGraw-Hill, New York, NY, 1998. 
[7]. Zhang Y, Aleman J, Arneri A, Bersini S, Piraino F, Shin S, Dokmeci M, Khademhosseini A, Biomed. Mater. 2015, 10, 034006.

[8]. Tomanek RJ, Runyan R, Formation of the Heart and its Regulation, Birkhäuser Boston, MA, 2012.

[9]. Zhang Y, Yue K, Aleman J, Moghaddam K, Bakht S, Dell'Erba V, Assawes P, Shin S, Dokmeci M, Oklu R, Khademhosseini A, Ann. Biomed. Eng. 2017, 45, 148. [PubMed: 27126775]

[10]. Langer R, Vacanti J, Vacanti C, Atala A, Freed L, Vunjak-Novakovic G, Tissue Eng. 1995, 1, 151. [PubMed: 19877924]

[11]. a) Rice JJ, Martino MM, De Laporte L, Tortelli F, Briquez PS, Hubbell JA, Adv. Healthc. Mater. 2012, 2, 57; [PubMed: 23184739] b) Pakulska M, Miersch S, Shoichet M, Science 2016, 351, 4750.

[12]. Bae H, Puranik A, Gauvin R, Edalat F, Carrillo-Conde B, Peppas N, Khademhosseini A, Sci. Transl. Med. 2012, 4, 160ps23.

[13]. Lovett M, Lee K, Edwards A, Kaplan D, Tissue Eng. Part B: Rev. 2009, 15, 353. [PubMed: 19496677]

[14]. Zhang Y, Choi S, Xia Y, Soft Matter 2013, 9, 9747; Wade R, Burdick J, Nano Today 2014, 9, 722.

[15]. a) Khademhosseini A, Langer R, Biomaterials 2007, 28, 5087; [PubMed: 17707502] b) Nichol J, Khademhosseini A, Soft matter 2009, 5, 1312; [PubMed: 20179781] c) Todhunter M, Jee N, Hughes A, Coyle M, Cerchiari A, Farlow J, Garbe J, LaBarge M, Desai T, Gartner Z, Nat. Meth. 2015, 12, 975.

[16]. a) Du Y, Lo E, Ali S, Khademhosseini A, PNAS 2008, 105, 9522; [PubMed: 18599452] b) Qi H, Ghodousi M, Du Y, Grun C, Bae H, Yin P, Khademhosseini A, Nat. Commun. 2013, 4, 2275. [PubMed: 24013352]

[17]. a) Onoe H, Okitsu T, Itou A, Kato-Negishi M, Gojo R, Kiriya D, Sato K, Miura S, Iwanaga S, Kuribayashi-Shigetomi K, Matsunaga Y, Shimoyama Y, Takeuchi S, Nat. Mater. 2013, 12, 584; [PubMed: 23542870] b) Tamayol A, Akbari M, Annabi N, Paul A, Khademhosseini A, Juncker D, Biotechnol. Adv. 2013, 31, 669; [PubMed: 23195284] c) Onoe H, Takeuchi S, Drug Discov. Today 2015, 20, 236. [PubMed: 25448757]

[18]. a) Yamato M, Okano T, Mater. Today 2004, 7, 42;b) Yang J, Yamato M, Kohno C, Nishimoto A, Sekine H, Fukai F, Okano T, Biomaterials 2005, 26, 6415; [PubMed: 16011847] c) Yang J, Yamato M, Sekine S, Sekiya S, Tsuda Y, Ohashi K, Shimizu T, Okano T, Adv. Mater. 2009, 21, 3404. [PubMed: 20882505]

[19]. Owen S, Shoichet M, J. Biomed. Mater. Res. A 2010, 94A, 1321.

[20]. a) Matsunaga Y, Morimoto Y, Takeuchi S, Adv. Mater. 2011, 23, 90; [PubMed: 20972982] b) Oliveira N, Zhang Y, Ju J, Chen A, Chen Y, Sonkusale S, Dokmeci M, Reis R, Mano J, Khademhosseini A, Chem. Mat. 2016, 28, 3641.

[21]. a) Kang E, Jeong G, Choi Y, Lee K, Khademhosseini A, Lee S, Nat. Mater. 2011, 10, 877; [PubMed: 21892177] b) Leong M, Toh J, Du C, Narayanan K, Lu H, Lim T, Wan A, Ying J, Nat. Commun. 2013, 4, 2353. [PubMed: 23955534]

[22]. Malda J, Visser J, Melchels F, Jüngst T, Hennink W, Dhert W, Groll J, Hutmacher D, Adv. Mater. 2013, 25, 5011. [PubMed: 24038336]

[23]. Murphy S, Atala A, Nat. Biotech. 2014, 32, 773.

[24]. Sheth R, Balesh E, Zhang Y, Hirsch J, Khademhosseini A, Oklu R, J. Vasc. Interv. Radiol. 2016, 27, 859. [PubMed: 27117948]

[25]. Hull CW, US Patents 1986.

[26]. Klimek L, Klein H, Schneider W, Mösges R, Schmelzer B, Voy E, Acta Otorhinolaryngol. Belg. 1993, 47, 329. [PubMed: 8213143]

[27]. Mandrycky C, Wang Z, Kim K, Kim D, Biotechnol. Adv. 2015, 34, 422. [PubMed: 26724184]

[28]. Raman R, Bhaduri B, Mir M, Shkumatov A, Lee M, Popescu G, Kong H, Bashir R, Adv. Healthc. Mater. 2015, 5, 610. [PubMed: 26696464]

[29]. Kyle S, Jessop Z, Al-Sabah A, Whitaker I, Adv. Healthc. Mater. 2017, 6, 1700264.

[30]. Boland T, Wilson W, Xu T, US Patents 2006.

[31]. Wilson W, Boland T, Anat. Rec. A Discov. Mol. Cell. Evol. Biol. 2003, 272, 491. [PubMed: 12740942] 
[32]. Gao G, Schilling A, Hubbell K, Yonezawa T, Truong D, Hong Y, Dai G, Cui X, Biotechnol. Lett. 2015, 37, 2349. [PubMed: 26198849]

[33]. a) Chang C, Boland E, Williams S, Hoying J, J. Biomed. Mater. Res. B 2011, 98, 160;b) Murphy S, Skardal A, Atala A, J. Biomed. Mater. Res. A 2013, 101A, 272.

[34]. Christensen K, Xu C, Chai W, Zhang Z, Fu J, Huang Y, Biotechnol. Bioeng. 2015, 112, 1047 [PubMed: 25421556]

[35]. Yoon S, Park J, Lee H, Yoon W, Hwang D, Jung S, Adv. Healthc. Mater. 2018, 7, e1800050.

[36]. Bohandy J, Kim B, Adrian F, J. Appl. Phys. 1986, 60, 1538.

[37]. Duocastella M, Colina M, Fernández-Pradas J, Serra P, Morenza J, Appl. Surf. Sci. 2007, 253, 7855.

[38]. a) Ali M, Pages E, Ducom A, Fontaine A, Guillemot F, Biofabrication 2014, 6, 045001;b) Guillemot F, Souquet A, Catros S, Guillotin B, Lopez J, Faucon M, Pippenger B, Bareille R, Remy M, Bellance S, Chabassier P, Fricain J, Amedee J, Acta Biomater. 2010, 6, 2494. [PubMed: 19819356]

[39]. Guillotin B, Souquet A, Catros S, Duocastella M, Pippenger B, Bellance S, Bareille R, Remy M, Bordenave L, Amedee J, Guillemot F, Biomaterials 2010, 31, 7250. [PubMed: 20580082]

[40]. Iwan Z, Hutmacher D, Tanc K, Teoha S, Biomaterials 2002, 23, 1169. [PubMed: 11791921]

[41]. Ozbolat I, Hospodiuk M, Biomaterials 2016, 76, 321. [PubMed: 26561931]

[42]. Fielding G, Bandyopadhyay A, Bose S, Dent. Mater. 2012, 28, 113 [PubMed: 22047943]

[43]. Li D, Xia Y, Adv. Mater. 2004, 16, 1151.

[44]. Huang Z, Zhang Y, Kotaki M, Ramakrishna S, Compos. Sci. Technol. 2003, 63, 2223.

[45]. a) Liu W, Huang C, Jin X, Nanoscale Res. Lett. 2015, 10, 949; [PubMed: 26055481] b) Huang C, Tang Y, Liu X, Sutti A, Ke Q, Mo X, Wang X, Morsi Y, Lin T, Soft Matter 2011, 7, 10812;c) Xie J, Li X, Xia Y, Macromol. Rapid. Commun. 2008, 29, 1775; [PubMed: 20011452] d) Liu W, Thomopoulos S, Xia Y, Adv. Healthc. Mater. 2012, 1, 10. [PubMed: 23184683]

[46]. Sun D, Chang C, Li S, Lin L, Nano Lett. 2006, 6, 839. [PubMed: 16608294]

[47]. Bisht G, Canton G, Mirsepassi A, Kulinsky L, Oh S, Dunn-Rankin D, Madou M, Nano Lett. 2011, 11, 1831. [PubMed: 21446719]

[48]. An B, Kim K, Lee H, Kim S, Shim Y, Lee D, Song J, Park J, Adv. Mater. 2015, 27, 4322. [PubMed: 26095718]

[49]. a) de Ruijter M, Hrynevich A, Haigh J, Hochleitner G, Castilho M, Groll J, Malda J, Dalton P, Small 2018, 14, 1702773;b) Castilho M, Feyen D, Flandes-Iparraguirre M, Hochleitner G, Groll J, Doevendans P, Vermonden T, Ito K, Sluijter J, Malda J, Adv. Healthc. Mater. 2017, 6, 1700311; cCastilho M, Hochleitner G, Wilson W, van Rietbergen B, Dalton P, Groll J, Malda J, Ito K, Sci. Rep. 2018, 8, 1245. [PubMed: 29352189]

[50]. Hochleitner G, Jüngst T, Brown T, Hahn K, Moseke C, Jakob F, Dalton P, Groll J, Biofabrication 2015, 7, 035002.

[51]. Brown T, Dalton P, Hutmacher D, Adv. Mater. 2011, 23, 5651. [PubMed: 22095922]

[52]. a) Dalton P, Joergensen N, Groll J, Moeller M, Biomed. Mater. 2008, 3, 034109;b) Buttafoco L, Kolkman N, Engbers-Buijtenhuijs P, Poot A, Dijkstra P, Vermes I, Feijen J, Biomaterials 2006, 27, 724. [PubMed: 16111744]

[53]. a) Zhang H, Xing T, Yin R, Shi Y, Yang S, Zhang W, Chin J. Traumatol. 2016, 19, 187; [PubMed: 27578372] b) Li J, Chen M, Fan X, Zhou H, J. Transl. Med. 2016, 14, 271. [PubMed: 27645770]

[54]. Panwar A, Tan L, Molecules 2016, 21, 3390.

[55]. Hospodiuk M, Dey M, Sosnoski D, Ozbolat I, Biotechnol. Adv. 2017, 35, 217. [PubMed: 28057483]

[56]. Axpe E, Oyen M, Int. J. Mol. Sci. 2016, 17, 1976.

[57]. Ji S, Guvendiren M, Front. Bioeng. Biotechnol. 2017, 5, 00023.

[58]. Ooi H, Mota C, Ten Cate A, Calore A, Moroni L, Baker M, Biomacromolecules 2018, 19, 3390. [PubMed: 29939754]

[59]. Lim K, Schon B, Mekhileri N, Brown G, Chia C, Prabakar S, Hooper G, Woodfield T, ACS Biomater. Sci. Eng. 2016, 2, 1752. 
[60]. Pan T, Song W, Cao X, Wang Y, J. Mater. Sci. Technol. 2016, 32, 889.

[61]. Yue K, Trujillo-de Santiago G, Alvarez M, Tamayol A, Annabi N, Khademhosseini A, Biomaterials 2015, 73, 254. [PubMed: 26414409]

[62]. a) Ouyang L, Highley C, Rodell C, Sun W, Burdick J, ACS Biomater. Sci. Eng. 2016, 2, 1743;b) Law N, Doney B, Glover H, Qin Y, Aman Z, Sercombe T, Liew L, Dilley R, Doyle B, J. Mech. Behav. Biomed. Mater. 2018, 77, 389; [PubMed: 29017117] c) Petta D, Armiento A, Grijpma D, Alini M, Eglin D, D'Este M, Biofabrication 2018, 10, 0441014.

[63]. Pati F, Jang J, Ha D, Won Kim S, Rhie J, Shim J, Kim D, Cho D, Nat. Commun. 2014, 5, 3935. [PubMed: 24887553]

[64]. Kang H, Lee S, Ko I, Kengla C, Yoo J, Atala A, Nat. Biotechnol. 2016, 34, 312. [PubMed: 26878319]

[65]. Lin X, Shi Y, Cao Y, Liu W, Biomed Mater. 2016, 11, 014109; Yang J, Zhang Y, Yue K, Khademhosseini A, Acta Biomater. 2017, 57, 1. [PubMed: 28088667]

[66]. Zhang Y, Yue K, Aleman J, Mollazadeh-Moghaddam K, Bakht S, Yang J, Jia W, Dell'Erba V, Assawes P, Shin S, Dokmeci M, Oklu R, Khademhosseini A, Ann. Biomed. Eng. 2016, 45, 148. [PubMed: 27126775]

[67]. Jungst T, Smolan W, Schacht K, Scheibel T, Groll J, Chem. Rev. 2016, 116, 1496. [PubMed: 26492834]

[68]. Schuurman W, Levett P, Pot M, van Weeren P, Dhert W, Hutmacher D, Melchels F, Klein T, Malda J, Macromol. Biosci. 2013, 13, 551. [PubMed: 23420700]

[69]. Colosi C, Shin S, Manoharan V, Massa S, Constantini M, Barbetta A, Dokmeci M, Dentini M, Khademhosseini A, Adv. Mater. 2016, 28, 677. [PubMed: 26606883]

[70]. Xavier J, Thakur T, Desai P, Jaiswal M, Sears N, Cosgriff-Hernandez E, Kaunas R, Gaharwar A, ACS Nano 2015, 9, 3109. [PubMed: 25674809]

[71]. Billiet T, Gevaert E, De Schryver T, Cornelissen M, Dubruel P, Biomaterials 2014, 35, 49. [PubMed: 24112804]

[72]. Mouser V, Melchels F, Visser J, Dhert W, Gawlitta D, Malda J, Biofabrication 2016, 8, 035003.

[73]. Engler A, Sen S, Sweeney H, Discher D, Cell 2006, 126, 677. [PubMed: 16923388]

[74]. Khalil S, Sun W, J. Biomech. Eng. 2009, 131, 111002.

[75]. Aguado B, Mulyasasmita W, Su J, Lampe K, Heilshorn S, Tissue Eng. Part A 2011, 18, 806. [PubMed: 22011213]

[76]. Blaeser A, Duarte Campos D, Puster U, Richtering W, Stevens M, Fischer H, Adv. Healthc. Mater. 2016, 5, 326. [PubMed: 26626828]

[77]. Liu W, Heinrich M, Zhou Y, Akpek A, Hu N, Liu X, Guan X, Zhong Z, Jin X, Khademhosseini A, Zhang Y, Adv. Healthc. Mater. 2017, 6, 1601451.

[78]. Turnbull G, Clarke J, Picard F, Riches P, Jia L, Han F, Li B, Shu W, Bioact Mater. 2017, 3, 278. [PubMed: 29744467]

[79]. a) Schuurman W, Khristov V, Pot M, van Weeren P, Dhert W, Malda J, Biofabrication 2011, 3, 021001;b) Xu T, Binder K, Albanna M, Dice D, Zhao W, Yoo J, Atala A, Biofabrication 2013, 5, 015001 .

[80]. Visser J, Melchels F, Jeon J, van Bussel E, Kimpton L, Byrne H, Dhert W, Dalton P, Hutmacher D, Malda J, Nat. Commun. 2015, 6, 6933. [PubMed: 25917746]

[81]. Hong S, Sycks D, Chan H, Lin S, Lopez G, Guilak F, Leong K, Zhao X, Adv. Mater. 2015, 27 , 4035. [PubMed: 26033288]

[82]. Sun T, Kurokawa T, Kuroda S, Ihsan A, Akasaki T, Sato K, Haque M, Nakajima T, Gong J, Nat. Mater. 2013, 12, 932. [PubMed: 23892784]

[83]. Poldervaart M, Goversen B, de Ruijter M, Abbadessa A, Melchels F, Öner F, Dhert W, Vermonden T, Alblas J, Plos One 2017, 12, e0177628.

[84]. Bendtsen S, Quinnell S, Wei M, J. Biomed. Mater. Res. A 2017, 105, 1457. [PubMed: 28187519]

[85]. Kyburz K, Anseth K, Ann. Biomed. Eng. 2015, 43, 489. [PubMed: 25753017]

[86]. Crapo P, Gilbert T, Badylak S, Biomaterials 2011, 32, 3233. [PubMed: 21296410] 
[87]. a) Aamodt J, Grainger D, Biomaterials 2016, 86, 68; [PubMed: 26890039] b) Balestrini J, Gard A, Gerhold K, Wilcox E, Liu A, Schwan J, Le A, Baevova P, Dimitrievska S, Zhao L, Sundaram S, Sun H, Rittie L, Dyal R, Broekelmann T, Mecham R, Schwartz M, Niklason L, White E, Biomaterials 2016, 102, 220; [PubMed: 27344365] c) Bautista C, Park H, Mazur C, Aaron R, Bilgen B, Plos One 2016, 11, e0158976;d) Helal-Neto E, Brandao-Costa R, Saldanha-Gama R, Ribeiro-Pereira C, Midlej V, Benchimol M, Morandi V, Barja-Fidalgo C, J. Cell. Physiol. 2016, 231, 2464. [PubMed: 27420801]

[88]. Bi H, Ming L, Cheng R, Luo H, Zhang Y, Jin Y, Tissue Eng J Regen. Med. 2016, 11, 2685.

[89]. Nagao R, Xu J, Luo P, Xue J, Wang Y, Kotha S, Zeng W, Fu X, Himmelfarb J, Zheng Y, Tissue Eng. Part A 2016, 22, 1140. [PubMed: 27481445]

[90]. Choudhury D, Tun H, Wang T, Naing M, Trends Biotechnol. 2018, 36, 741. [PubMed: 29891181]

[91]. Jang J, Kim T, Kim B, Kim S, Kwon S, Cho D, Acta Biomater. 2016, 33, 88. [PubMed: 26774760]

[92]. Jang J, Park H, Kim S, Kim H, Park J, Na S, Kim H, Park M, Choi S, Park S, Kim S, Kwon S, Biomaterials 2017, 112, 264. [PubMed: 27770630]

[93]. Toprakhisar B, Nadernezhad A, Bakirci E, Khani N, Skvortsov G, Koc B, Macromol. Biosci. 2018, 18, e1800024.

[94]. a) Dickey M, Adv. Mater. 2017, 29, 1606425;b) Xu Y, Wu X, Guo X, Kong B, Zhang M, Qian X, Mi S, Sun W, Sensors (Basel) 2017, 17, e1166.

[95]. Singh M, Haverinen H, Dhagat P, Jabbour G, Adv. Mater. 2010, 22, 673. [PubMed: 20217769]

[96]. Cummins G, Desmulliez M, Circuit World 2012, 38, 193.

[97]. Sridhar A, Blaudeck T, Baumann R, Material Matters 2011, 6, 12.

[98]. Park S, Vosguerichian M, Bao Z, Nanoscale 2013, 5, 1727. [PubMed: 23381727]

[99]. Kamyshny A, Magdassi S, Small 2014, 10, 3515. [PubMed: 25340186]

[100]. Shanmugasundaram T, Radhakrishnan M, Gopikrishnan V, Kadirvelu K, Balagurunathan R, Nanoscale 2017, 9, 16773. [PubMed: 29072767]

[101]. Zhu K, Shin S, van Kempen T, Li Y, Ponraj V, Nasajpour A, Mandla S, Hu N, Liu X, Leijten J, Lin Y, Hussain M, Zhang Y, Tamayol A, Khademhosseini A, Adv. Funct. Mater. 2017, 27, 1605352.

[102]. a) Torrisi F, Hasan T, Wu W, Sun Z, Lombardo A, Kulmala T, Hsieh G, Jung S, Bonaccorso F, Paul P, ACS Nano 2012, 6, 2992; [PubMed: 22449258] b) Parviz D, Das S, Ahmed H, Irin F, Bhattacharia S, Green M, ACS Nano 2012, 6, 8857; [PubMed: 23002781] c) Hasan T, Torrisi F, Sun Z, Popa D, Nicolosi V, Privitera G, Bonaccorso F, Ferrari A, Phys. Status Solidi B 2010, 247, 2953; d) Wajid A, Das S, Irin F, Ahmed H, Shelburne J, Parviz D, Fullerton R, Jankowski A, Hedden R, Green M, Carbon 2012, 50, 526.

[103]. Dai B, Fu L, Liao L, Liu N, Yan K, Chen Y, Liu Z, Nano Research 2011, 4, 434.

[104]. a) Huang L, Huang Y, Liang J, Wan X, Chen Y, Nano Research 2011, 4, 675;b) Le L, Ervin M, Qiu H, Fuchs B, Lee W, Electrochem. Commun. 2011, 13, 355;c) Kong D, Le L, Li Y, Zunino J, Lee W, Langmuir 2012, 28, 13467. [PubMed: 22924965]

[105]. Jakus AE, Secor EB, Rutz AL, Jordan SW, Hersam MC, Shah RN, ACS Nano 2015, 9, 4636. [PubMed: 25858670]

[106]. Huang C, Shrestha L, Ariga K, Hsu S, J. Mater. Chem. B 2017, 5, 8854.

[107]. Okimoto H, Takenobu T, Yanagi K, Miyata Y, Shimotani H, Kataura H, Iwasa Y, Adv. Mater. 2010, 22, 3981. [PubMed: 20730813]

[108]. Hecht D, Hu L, Irvin G, Adv. Mater. 2011, 23, 1482; [PubMed: 21322065] Hu L, Hecht D, Gruner G, Chem. Rev. 2010, 110, 5790. [PubMed: 20939616]

[109]. a) Small W, Small 2007, 3, 1500; [PubMed: 17668430] b) Mionić M, Pataky K, Gaal R, Magrez A, Brugger J, Forró L, J. Mater. Chem. 2012, 22, 14030.

[110]. Denneulin A, Bras J, Carcone F, Neuman C, Blayo A, Carbon 2011, 49, 2603.

[111]. a) Islam M, Rojas E, Bergey D, Johnson A, Yodh A, Nano Lett. 2003, 3, 269;b) Wang H, Zhou W, Ho D, Winey K, Fischer J, Glinka C, Hobbie E, Nano Lett. 2004, 4, 1789;c) Whitssitt E, Barron A, Nano Lett. 2003, 3, 775. 
[112]. a) Azoubel S, Shemesh S, Magdassi S, Nanotechnology 2012, 23, 344003;b) Kordás K, Mustonen T, Tóth G, Jantunen H, Lajunen M, Soldano C, Talapatra S, Kar S, Vajtai R, Ajayan P, Small 2006, 2, 1021; [PubMed: 17193162] c) Lee Y, Kim S, Lee K, Myung N, Choa Y, Thin Solid Films 2013, 536, 160;d) Wei T, Ruan J, Fan Z, Luo G, Wei F, Carbon 2007, 45, 2712.

[113]. Shin S, Farzad R, Tamayol A, Manoharan V, Mostafalu P, Zhang Y, Akbari M, Jung S, Kim D, Comotto M, Annabi N, Al-Hazmi F, Dokmeci M, Khademhosseini A, Adv. Mater. 2016, 28, 3280. [PubMed: 26915715]

[114]. Lu W, Liu Y, Gou J, Leng J, Du S, Smart Mater. Struct. 2010, 19, 075021.

[115]. Xu L, Gutbrod S, Bonifas A, Su Y, Sulkin M, Lu N, Chung H, Jang K, Liu Z, Ying M, Lu C, Webb R, Kim J, Laughner J, Cheng H, Liu Y, Ameen A, Jeong J, Kim G, Huang Y, Efimov I, Rogers J, Nat. Commun. 2014, 5, 3329. [PubMed: 24569383]

[116]. Muth J, Vogt D, Truby R, Mengüç Y, Kolesky D, Wood R, Lewis J, Adv. Mater. 2014, 26, 6307. [PubMed: 24934143]

[117]. Jae Sung Park TKWSK, Sci. Rep. 2017, 7, 3246; [PubMed: 28607350] Athreya S, Kalaitzidou K, Das S, Mater. Sci. Eng. 2010, 527, 2637.

[118]. Lind J, Busbee T, Valentine A, Pasqualini F, Yuan H, Yadid M, Park S, Kotikian A, Nesmith A, Campbell P, Vlassak J, Lewis J, Parker K, Nat. Mater. 2017, 16, 303. [PubMed: 27775708]

[119]. Krassenstein B, 3DPrint.com, accessed on January 30th 2015.

[120]. Ullah I, Subbarao R, Rho G, Biosci. Rep. 2015, 35, e00191.

[121]. Costantini M, Idaszek J, Szoke K, Jaroszewicz J, Dentini M, Barbetta A, Brinchmann J, Swieszkowski W, Biofabrication 2016, 8, 035002.

[122]. Gao G, Zhang X, Hubbell K, Cui X, Biotechnol. Bioeng. 2016, 114, 208; [PubMed: 27345768] Yu C, Tang K, Suh J, Jiang R, Tsai S, Tsai M, Development 2012, 139, 2330. [PubMed: 22669823]

[123]. Duarte Campos D, Blaeser A, Buellesbach K, Sen K, Xun W, Tillmann W, Fischer H, Adv. Healthc. Mater. 2016, 5, 1336. [PubMed: 27072652]

[124]. Byambaa B, Annabi N, Yue K, Trujillo-de Santiago G, Alvarez M, Jia W, Kazemzadeh-Narbat M, Shin S, Tamayol A, Khademhosseini A, Adv. Healthc. Mater. 2017, 6, 1700015.

[125]. Minteer D, Marra K, Rubin J, Adv. Biochem. Eng. Biotechnol. 2013, 129, 59. [PubMed: 22825719]

[126]. Kang L, Armstrong P, Lee L, Duan B, Kang K, Butcher J, Ann. Biomed. Eng. 2016, 45, 360. [PubMed: 27106636]

[127]. Gruene M, Pflaum M, Deiwick A, Koch L, Schlie S, Unger C, Wilhelmi M, Haverich A, Chichkov B, Biofabrication 2011, 3, 015005.

[128]. Xu T, Zhao W, Zhu J, Albanna M, Yoo J, Atala A, Biomaterials 2013, 34, 130. [PubMed: 23063369]

[129]. Joo S, Ko I, Atala A, Yoo J, Lee S, Arch. Pharmacal. Research 2012, 35, 271.

[130]. Clarke D, Johansson C, Wilbertz J, Veress B, Nilsson E, Karlström H, Lendahl U, Frisén J, Science 2000, 288, 1660. [PubMed: 10834848]

[131]. Murnagham I, Pluripotent Stem Cells, Available at: Explore Stem Cells, accessed January 30th 2017.

[132]. Ouyang L, Yao R, Mao S, Chen X, Na J W Sun, Biofabrication 2015, 7, 044101.

[133]. Faulkner-Jones A, Fyfe C, Cornelissen D, Gardner J, King J, Courtney A, Shu W, Biofabrication 2015, 7, 044102.

[134]. a) Lo B, Parham L, Endocr. Rev. 2009, 30, 204; [PubMed: 19366754] b) Thomson J, ItskovitzEldor J, Shapiro S, Waknitz M, Swiergiel J, Marshall V, Jones J, Science 1998, 282, 1145; [PubMed: 9804556] c) Allum N, Allansdottir A, Gaskell G, Hampel J, Jackson J, Moldovan A, Priest S, Stares S, Stoneman P, Plos One 2017, 12, e0176274.

[135]. Ma X, Qu X, Zhu W, Li Y, Yuan S, Zhang H, Liu J, Wang P, Lai C, Zanella F, Feng G, Sheikh F, Chien S, Chen S, PNAS 2015, 113, 2206.

[136]. Gu Q, Tomaskovic-Crook E, Wallace G, Crook J, Adv. Healthc. Mater. 2017, 6, 1700175.

[137]. Ren X, Wang F, Chen C, Gong X, Yin L, Yang L, BMC Musculoskelet. Disord. 2016, 17, 301. [PubMed: 27439428] 
[138]. Nguyen D, Funk J, Robbins J, Crogan-Grundy C, Presnell S, Singer T, Roth A, Plos One 2016, 11, e0158674.

[139]. Godoy P, Hoewitt N, Albrecht U, Andersen M, Ansari N, Bhattacharya S, Bode J, Bolleyn J, Borner C, Böttger J, Braeuning A, Budinsky R, Burkhardt B, Cameron N, Camussi G, Cho C, Choi Y, Craig Rowlands J, Dahmen U, Damm G, Dirsch O, Donato M, Dong J, Dooley S, Drasdo D, Eakins R, Ferreira K, Fonsato V, Fraczek J, Gebhardt R, Gibson A, Glanemann M, Goldring C, Gómez-Lechón M, Groothuis G, Gustavsson L, Guyot C, Hallifax D, Hammad S, Hayward A, Häussinger D, Hellerbrand C, Hewitt P, Hoehme S, Holzhütter H, Houston J, Hrach J, Ito K, Jaeschke H, Keitel V, Kelm J, Kevin Park B, Kordes C, Kullak-Ublick G, LeCluyse E, Lu P, Luebke-Wheeler J, Lutz A, Maltman D, Matz-Soja M, McMullen P, Merfort I, Messner S, Meyer C, Mwinyi J, Naisbitt D, Nussler A, Olinga P, Pampaloni F, Pi J, Pluta L, Przyborski S, Ramachandran A, Rogiers V, Rowe C, Schelcher C, Schmich K, Schwarz M, Singh B, Stelzer E, Stieger B, Stöber R, Sugiyama Y, Tetta C, Thasler W, Vanhaecke T, Vinken M, Weiss T, Widera A, Woods C, Xu J, Yarborough K, Hengstler J, Arch. Toxicol. 2013, 87, 1315. [PubMed: 23974980]

[140]. Kolesky D, Homan K, Skylar-Scott M, Lewis J, PNAS 2016, 113, 3179. [PubMed: 26951646]

[141]. Marieb E, Hoehn K, Human Anatomy \& Physiology, Pearson International Edition, San Francisco, CA, 2010, Eigth Edition.

[142]. Purves D, Augustine G, Fitzpatrick D, Hall W, LaMantia A, McNamara J, White L, Neuroscience, Sinauer Assocites Inc Sunderland, MA, 2012, 5th Edition.

[143]. Ligon S, Liska R, Stampfl J, Gurr M, Mülhaupt R, Chem. Rev. 2017, 117, 10212. [PubMed: 28756658]

[144]. Highley C, Rodell C, Burdick J, Adv. Mater. 2015, 27, 5075. [PubMed: 26177925]

[145]. Hinton T, Jallerat Q, Palchesko R, Park J, Grodzicki M, Shue H, Ramadan M, Hudson A, Feinberg A, Sci. Adv. 2015, 1, e1500758.

[146]. Bhattacharjee T, Zehnder S, Rowe K, Jain S, Nixon R, Sawyer W, Angelini T, Sci. Adv. 2015, 1, e1500655.

[147]. Jin Y, Compaan A, Bhattacharjee T, Huang Y, Biofabrication 2016, 8, 025016.

[148]. Gauvin R, Chen Y, Lee J, Soman P, Zorlutuna P, Nichol J, Bae H, Chen S, Khademhosseini A, Biomaterials 2012, 33, 3824. [PubMed: 22365811]

[149]. Zhang A, Qu X, Soman P, Hribar K, Lee J, Chen S, He S, Adv. Mater. 2012, 24, 4266. [PubMed: 22786787]

[150]. Zhu W, Holmes B, Glazer R, Zhang L, Nanomedicine 2015, 12, 69. [PubMed: 26472048]

[151]. Soman P, Chung P, Zhang A, Chen S, Biotechnol. Bioeng. 2013, 110, 3038. [PubMed: 23686741]

[152]. Shanjani Y, Pan C, Elomaa L, Yang Y, Biofabrication 2015, 7, 045008.

[153]. Bajaj P, Marchwiany D, Duarte C, Bashir R, Adv. Healthc. Mater. 2013, 2, 450. [PubMed: 23463644]

[154]. Cvetkovic C, Raman R, Chan V, Williams B, Tolish M, Bajaj P, Sakar M, Asada H, Saif M, Bashir R, PNAS 2014, 111, 10125. [PubMed: 24982152]

[155]. Lim K, Levato R, Costa P, Castilho M, Alcala-Orozco C, van Dorenmalen K, Melchels F, Gawlitta D, Hooper G, Malda J, Woodfield T, Biofabrication 2018, 10, 034101.

[156]. Gou M, X Qu X, Zhu W, Xiang M, Yang J, Zhang K, Wei Y, Chen S, Nat. Commun. 2014, 5, 3774. [PubMed: 24805923]

[157]. Zhu W, Li J, Leong Y, Rozen I, Qu X, Dong R, Wu Z, Gao W, Chung P, Wang J, Chen S, Adv. Mater. 2015, 27, 4411. [PubMed: 26121113]

[158]. Tumbleston J, Shirvanyants D, Ermoshkin N, Janusziewicz R, Johnson A, Kelly D, Chen K, Pinschmidt R, Rolland J, Ermoshkin A, Samulski E, DeSimone J, Science 2015 347, 1349 [PubMed: 25780246]

[159]. Hirt L, Ihle S, Pan Z, Dorwling-Carter L, Reiser A, Wheeler J, Spolenak R, Voros J, Zambelli T, Adv. Mater. 2016, 28, 2311. [PubMed: 26783090]

[160]. Zhang Y, Oklu R, Dokmeci M, Khademhosseini A, Cold Spring Harb. Perspect. Med. 2017, 8, 025718 . 
[161]. Hanson Shepherd J, Parker S, Shepherd R, Gillette M, Lewis J, Nuzzo R, Adv. Funct. Mater. 2011, 21, 47. [PubMed: 21709750]

[162]. Huang C, Chen S, Lai C, Reneker D, Qiu H, Ye Y, Hou H, Nanotechnology 2006, 17, 1558. [PubMed: 26558558]

[163]. Reneker D, Yarin A, Polymer 2008, 49, 2387.

[164]. Farrugia BL, Brown T, Upton Z, Hutmacher D, Dalton P, Dargaville T, Biofabrication 2013, 5, 025001.

[165]. Chang C, Limkrailassiri K, Lin L, Appl. Phys. Lett. 2008, 93, 123111.

[166]. Brown T, Edin F, Detta N, Skelton A, Hutmacher D, Dalton P, Mater. Sci. Eng. C 2014, 45, 698.

[167]. Wade R, Bassin E, Gramlich W, Burdick J, Adv. Mater. 2015, 27, 1356. [PubMed: 25640972]

[168]. Wunner F, Wille M, Noonan T, Bas O, Dalton P, De-Juan-Pardo E, Hutmacher D, Adv. Mater. 2018, 30, 1706570.

[169]. a) Guo J, Li C, Ling S, Huang W, Chen Y, Kaplan D, Biomaterials 2017, 145, 44; [PubMed: 28843732] b) Cross L, Shah K, Palani S, Peak C, Gaharwar A, Nanomedicine 2017, 14, 2465. [PubMed: 28554596]

[170]. Liu H, Yang L, Zhang E, Zhang R, Cai D, Zhu S, Ran J, Bunpetch V, Cai Y, Heng B, Hu Y, Dai X, Chen X, Ouyang H, Acta Biomater. 2017, 56, 129. [PubMed: 28502669]

[171]. Li X, Cheng R, Sun Z, Su W, Pan G, Zhao S, Zhao J, Cui W, Acta Biomater. 2017, 61, 204. [PubMed: 28778532]

[172]. a) Spalazzi J, Doty S, Moffat K, Levine W, Lu H, Tissue Eng. 2006, 12, 3497; [PubMed: 17518686] b) Wang I, Shan J, Choi R, Oh S, Kepler C, Chen F, Lu H, J. Orthop. Res. 2007, 25, 1609; [PubMed: 17676622] c) Lu H, Subramony S, Boushell M, Zhang X, Ann. Biomed. Eng. 2010, 38, 2142; [PubMed: 20422291] d) Atesok K, Doral M, Karlsson J, Egol K, Jazrawi L, Coelho P, Martinez A, Matsumoto T, Owens B, Ochi M, Hurwitz S, Atala A, Fu F, Lu H, Rodeo S, Knee Surg. Sports Traumatol. Arthrosc. 2014, 24, 2365; [PubMed: 25466277] e) Lu H, Thomopoulos S, Annu. Rev. Biomed. Eng. 2013, 15, 201; [PubMed: 23642244] f) Liu W, Lipner J, Xie J, Manning C, Thomopoulos S, Xia Y, ACS Appl. Mater. Interfaces 2014, 6, 2842. [PubMed: 24433042]

[173]. Ober T, Foresti D, Lewis J, PNAS 2015, 112, 12293. [PubMed: 26396254]

[174]. Liu W, Zhang Y, Heinrich M, De Ferrari F, Jang H, Bakht S, Alvarez M, Yang J, Li Y, Trujillode Santiago G, Miri A, Zhu K, Khoshakhlagh P, Prakash G, Cheng H, Guan X, Zhong Z, Ju J, Zhu G, Jin X, Shin S, Dokmeci M, Khademhosseini A, Adv. Mater. 2017, 29, 1604630.

[175]. Kolesky D, Truby R, Gladman A, Busbee T, Homan K, Lewis J, Adv. Mater. 2014, $26,3124$. [PubMed: 24550124]

[176]. Choi J, Ha Y, Lee S, Choi K, J. Mech. Sci. Technol. 2006, 20, 2094.

[177]. Choi J, Kim H, Wicker R, J. Mater. Process. Tech. 2011, 211, 318.

[178]. Zhou C, Chen Y, Yang Z, Khoshnevis B, Rapid Prototyp. J. 2013, $19,153$.

[179]. Han L, Suri S, Schmidt C, Chen S, Biomed. Microdevices 2010, 12, 721. [PubMed: 20393801]

[180]. Miri A, Nieto D, Iglesias L, Goodarzi Hosseinabadi H, Maharjan S, Ruiz-Esparza G, Khoshakhlagh P, Manbachi A, Dokmeci M, Chen S, Shin S, Zhang Y, Khademhosseini A, Adv. Mater. 2018, 30, e1800242.

[181]. Ozbolat IT, Trends Biotechnol. 2015, 33, 395. [PubMed: 25978871]

[182]. Rebelo S, Pinto C, Martins T, Harrer N, Estrada M, Loza-Alvarez P, Cabeçadas J, Alves P, Gualda E, Sommergruber W, Brito C, Biomaterials 2018, 163, 185. [PubMed: 29477032]

[183]. Onofrillo C, Duchi S, O’Connell C, Blanchard R, O’Connor A, Scott M, Wallace G, Choong P, Di Bella C, Biofabrication 2018, 10, 045006.

[184]. a) Zhao F, Wang J, Guo H, Liu S, He W, J. Nanomater. 2015, 2015, 893545;b) Evans N, Oreffo R, Healy E, Thurner P, YH M, J. Mech. Behav. Biomed. Mater. 2013, 28, 397; [PubMed: 23746929] c) Ghanavati Z, Neisi N, Bayati V, Makvandi M, Anat. Cell Biol. 2015, 48, 251. [PubMed: 26770875]

[185]. Skardal A, Mack D, Kapetanovic E, Atala A, Jackson J, Yoo J, Soker S, Stem Cells Transl. Med. 2012, 1, 792. [PubMed: 23197691] 
[186]. Skardal A, Murphy S, Crowell K, Mack D, Atala A, Soker S, J. Biomed. Mater. Res. B. Appl. Biomater. 2017, 105, 1986. [PubMed: 27351939]

[187]. Li L, Yu F, Shi J, Shen S, Teng H, Yang J, Wang X, Jiang Q, Sci. Rep. 2017, 7, 9416. [PubMed: 28842703]

[188]. Han Y, Hu J, Genin G, Lu T, Xu F, Sci. Rep. 2014, 6, 4872.

[189]. Di Bella C, Duchi S, O’Connell C, Blanchard R, Augustine C, Yue Z, Thompson F, Richards C, Beirne S, Onofrillo C, Bauquier S, Ryan S, Pivonka P, Wallace G, Choong P, Tissue Regen J Med. 2018, 12, 611.

[190]. Hakimi N, Cheng R, Leng L, Sotoudehfar M, Ba P, Bakhtyar N, Amini-Nik S, Jeschke M, Günther A, Lab Chip 2018, 8, 1440.

[191]. Alarçin E, Lee T, Karuthedom S, Mohammadi M, Brennan M, Lee D, Marrella A, Zhang J, Syla D, Zhang Y, Khademhosseini A, Jang H, Biomater Sci. 2018, 6, 1604. [PubMed: 29736522]

[192]. Li Y, Zhang Y, Akpek A, Shin S, Khademhosseini A, Biofabrication 2016, 9, 012001.

[193]. a) Kaur J, Spranger M, Hammond R, Krishnan A, Alvarez A, Augustyniak R, O’Leary D, Am. J. Physiol. Regul. Integr. Comp. Physiol. 2015, 308, 524;b) Senador D, Kaur J, Alvarez A, Hanna H, Krishnan A, Altamimi Y, O’Leary D, Am. J. Physiol. Regul. Integr. Comp. Physiol. 2017, 313, 29;c) Moreira-Rodrigues M, Mendes P, Graça A, Martinho R, Serrão P, Moura D, Life Sci. 2016, 156, 1. [PubMed: 27221021]

[194]. Tibbits S, Architectural Design 2014, 84, 116.

[195]. Ge Q, Qi H, Dunn M, Appl. Phys. Lett. 2013, 103, 131901.

[196]. An J, Chua C, Mironov V, Int. J. Bioprint. 2016, 2, 3.

[197]. a) Khoo Z, Teoh J, Liu Y, Chua C, Yang S, An J, Leong K, Yeong W, Virtual Phys. Prototyp. 2015, 10, 103;b) Choi J, Kwon O, Jo W, Lee H, Moon M, 3D Print. Addit. Manuf. 2015, 2, 159;c) Raviv D, Zhao W, McKnelly C, Papadopoulou A, Kadambi A, Shi B, Hirsch S, Dikovsky D, Zyracki M, Olguin C, Raskar R, Tibbits S, Sci. Rep. 2014, 4, 7422; [PubMed: 25522053] d)

Yu C, Duan Z, Yuan P, Li Y, Su Y, Zhang X, Pan Y, Dai L, Nuzzo R, Huang Y, Adv. Mater. 2013, 25, 1541. [PubMed: 23255239]

[198]. a) Tasoglu S, Diller E, Guven S, Sitti M, Demirci U, Nat. Commun. 2014, 5, 3124; [PubMed: 24469115] b) Zhou J, Sheiko S, J. Polym. Sci. B Polym. Phys. 2016, 54, 1365;c) Thérien-Aubin H, Wu Z, Nie Z, Kumacheva E, JACS 2013, 135, 4834;d) Wu Z, Moshe M, Greener J, TherienAubin H, Nie Z, Sharon E, Kumacheva E, Nat. Commun. 2013, 4, 1586; [PubMed: 23481394] e) Shim T, Kim S, Heo C, Jeon H, Yang S, Angew. Chem. Int. Ed. 2012, 51, 1420;f) Zarek M, Layani M, Cooperstein I, Sachyani E, Cohn D, Magdassi S, Adv. Mater. 2015, 28, 4449. [PubMed: 26402320]

[199]. a) Zhou Y, Huang W, Kang S, Wu X, Lu H, Fu J, Cui H, J. Mech. Sci. Technol. 2015, 29, 4281;b) Ionov L, Adv. Funct. Mater. 2013, 23, 4555;Ashammakhi N, Ahadian S, Zengjie F, Suthiwanich K, Lorestani F, Orive G, Ostrovidov S, Khademhosseini A, Biotechnol. J. 2018, 13, e1800148.

[200]. Miao S, Zhu W, Castro N, Nowicki M, Zhou X, Cui H, Fisher J, Zhang L, Sci. Rep. 2016, 6, 27226. [PubMed: 27251982]

[201]. Bakarich S, Gorkin R, Panhuis M, Spinks G, Macromol. Rapid. Commun. 2015, 36, 1211. [PubMed: 25864515]

[202]. Ge Q, Dunn C, Qi H, Dunn M, Smart Mater. Struct. 2014, 23, 094007.

[203]. Sydney Gladman A, Matsumoto E, Nuzzo R, Mahadevan L, Lewis J, Nat. Mater. 2016, 15, 413. [PubMed: 26808461]

[204]. Kim Y, Yuk H, Zhao R, Chester S, Zhao X, Nature 2018, 558, 274. [PubMed: 29899476]

[205]. Betsch M, Cristian C, Lin Y, Blaeser A, Schöneberg J, Vogt M, Buhl E, Fischer H, Duarte Campos D, Adv. Healthc. Mater. 2018, 7, e1800894.

[206]. Yoo S, Expert Opin. Ther. Pat. 2015, 25, 507. [PubMed: 25711801]

[207]. Nomi M, Atala A, De Coppi P, Soker S, Mol. Aspects. Med. 2002, 23, 463. [PubMed: 12385748]

[208]. a) Kirkpatrick C, Fuchs S, Unger R, Adv. Drug Deliv. Rev. 2011, 63, 291; [PubMed: 21281686] b) Unger R, Dohle E, Kirkpatrick C, Adv. Drug Deliv. Rev. 2015, 94, 116; [PubMed: 25817732] 
c) Nguyen L, Annabi N, Nikkhah M, Bae H, Binan L, Park S, Kang Y, Yang Y, Khademhosseini A, Tissue Eng. Part B: Rev. 2012, 18, 363; [PubMed: 22765012] d) Richardson T, Peters M, Ennett A, Mooney D, Nat. Biotech. 2001, 19, 1029;e) Oladipupo S, Hu S, Kovalski J, Yao J, Santeford A, Sohn R, Shohet R, Maslov K, Wang L, Arbeit J, PNAS 2011, 108, 13264.

[PubMed: 21784979]

[209]. Bertassoni L, Cecconi M, Manoharan V, Nikkhah M, Hjortnaes J, Cristino A, Barabaschi G, Demarchi D, Dokmeci M, Yang Y, Lab Chip 2014, 14, 2202. [PubMed: 24860845]

[210]. Lee V, Kim D, Ngo H, Lee Y, Seo L, Yoo S, Vincent P, Dai G, Biomaterials 2014, 35, 8092. [PubMed: 24965886]

[211]. Miller J, Stevens K, Yang M, Baker B, Nguyen D, Cohen D, Toro E, Chen A, Galie P, Yu X, Nat. Mater. 2012, 11, 768. [PubMed: 22751181]

[212]. Xu Y, Hu Y, Liu C, Yao H, Liu B, Mi S, Materials (Basel) 2018, 11, e1581.

[213]. Zhu W, Qu X, Zhu J, Ma X, Patel S, Liu J, Wang P, Lai C, Gou M, Xu Y, Zhang K, Chen S, Biomaterials 2017, 124, 106. [PubMed: 28192772]

[214]. Gao Q, He Y, Fu J, Liu A, Ma L, Biomaterials 2015, 61, 203. [PubMed: 26004235]

[215]. a) Luo Y, Lode A, Gelinsky M, Adv. Healthc. Mater. 2013, 2, 777; [PubMed: 23184455] b) Yu Y, Zhang Y, Martin J, Ozbolat I, Biomech Eng J 2013, 135, 91011;c) Zhang Y, Yu Y, Chen H, Ozbolat I, Biofabrication 2013, 5, 025004;d) Zhang Y, Yu Y, Akkouch A, Dababneh A, Dolati F, Ozbolat I, Biomater Sci. 2015, 3, 134; [PubMed: 25574378] e) Zhu K, Chen N, Liu X, Mu X, Zhang W, Wang C, Zhang Y, Macromol. Biosci. 2018, 18, e1800127.

[216]. Jia W, Gungor-Ozkerim P, Zhang Y, Yue K, Zhu K, Liu W, Pi Q, Byambaa B, Dokmeci M, Shin S, Khademhosseini A, Biomaterials 2016, 106, 58. [PubMed: 27552316]

[217]. Norotte C, Marga F, Niklason L, Forgacs G, Biomaterials 2009, 30, 5910. [PubMed: 19664819]

[218]. Kucukgul C, Ozler S, Inci I, Karakas E, Irmak S, Gozuacik D, Taralp A, Koc B, Biotechnol. Bioeng. 2015, 112.

[219]. Itoh M, Nakayama K, Noguchi R, Kamohara K, Furukawa K, Uchihashi K, Toda S, Oyama J, Node K, Morita S, PLoS One 2015, 10, e0136681.

[220]. Moldovan N, Hibino N, Nakayama K, Tissue Eng. Part B 2017, 23, 237.

[221]. Lozano R, Stevens L, Thompson B, Gilmore K, Gorkin R, Stewart E, Panhuis M, RomeroOrtega M, Wallace G, Biomaterials 2015, 67, 264. [PubMed: 26231917]

[222]. Pateman C, Harding A, Glen A, Taylor C, Christmas C, Robinson P, Rimmer S, Boissonade F, Claeyssens F, Haycock J, Biomaterials 2015, 49, 77. [PubMed: 25725557]

[223]. Lee S, Nowicki M, Harris B, Zhang L, Tissue Eng. Part A 2017, 23.

[224]. Kendroud S, Bhimji S, Physiology, Nociceptive Pathways. Available at StatPearls, accessed on January 30th 2017.

[225]. a) Wang C, Lu C, Peng J, Hu C, Wang Y, Neural Regen Res. 2017, 12;Hewson D, Bedforth N, Hardman J, Anaesthesia 2018, 73;b) Jellish WS, Oftadeh M, J. Cardiothorac. Vasc. Anesth. 2017, 32, 495. [PubMed: 29248326]

[226]. Zhu W, Harris B, Zhang L, Conf Proc IEEE Eng Med Biol Soc. 2016, 2016, 4185. [PubMed: 28269205]

[227]. Ning L, Sun H, Lelong T, Guilloteau R, Zhu N, Schreyer D, Chen X, Biofabrication 2018, 10, 035014.

[228]. a) Harre U, Schett G, Semin. Immunopathol. 2017, 39, 355; [PubMed: 28597065] b) Choi J, Yong K, Choi J, J. Cell Physiol. 2017, 233, 1913. [PubMed: 28542924]

[229]. Markstedt K, Mantas A, Tournier I, Martinez Avila H, Hagg D, Gatenholm P, Biomacromolecules 2015, 16, 1489. [PubMed: 25806996]

[230]. Kundu J, Shim J, Jang J, Kim S, Cho D, J. Tissue Regen. M. 2015, 9, 1286.

[231]. Holmes B, Bulusu K, Plesniak M, Zhang L, Nanotechnology 2016, 27, 064001.

[232]. Kang H, Lee S, Ko I, Kengla C, Yoo J, Atala A, Nat. Biotechnol. 2016, 34, 312. [PubMed: 26878319]

[233]. Keriquel V, Oliveira H, Rémy M, Ziane S, Delmond S, Rousseau B, Rey S, Catros S, Amédée J, Guillemot F, Fricain J, Sci. Rep. 2017, 7. 
[234]. Zhang Y, Arneri A, Bersini S, Shin S, Zhu K, Goli-Malekabadi Z, Aleman J, Colosi C, Busignani F, Dell'Erba V, Bishop C, Shupe T, Demarchi D, Moretti M, Rasponi M, Dokmeci M, Atala A, Khademhosseini A, Biomaterials 2016, 110, 45. [PubMed: 27710832]

[235]. Wu Y, Wang L, Guo B, Ma P, ACS Nano 2017, 11, 5646. [PubMed: 28590127]

[236]. Ong C, Fukunishi T, Zhang H, Huang C, Nashed A, Blazeski A, DiSilvestre D, Vricella L, Conte J, Tung L, Tomaselli G, Hibino N, Sci. Rep. 2017, 7, 4566. [PubMed: 28676704]

[237]. Maiullari F, Costantini M, Milan M, Pace V, Chirivì M, Maiullari S, Rainer A, Baci D, Marei H, Seliktar D, Gargioli C, Bearzi C, Rizzi R, Sci. Rep. 2018, 8, 13532. [PubMed: 30201959]

[238]. Costantini M, Test S, Mozetic P, Barbetta A, Fuoco C, Fornetti E, Tamiro F, Bernardini S, Jaroszewicz J, Święszkowski W, Trombetta M, Castagnoli L, Seliktar D, Garstecki P, Cesareni G, Cannata S, Rainer A, Gargioli C, Biomaterials 2017, 131, 98. [PubMed: 28388499]

[239]. Kim J, Seol Y, Ko I, Kang H, Lee Y, Yoo J, Atala A, Lee S, Sci. Rep. 2018, 8, 12307. [PubMed: 30120282]

[240]. Organovo, http://www.organovo.com/partnership/. Accessed on January 30th 2019.

[241]. a) Allevi, https://allevi3d.com/, Accessed on January 30th 2019.b) VWR, https://us.vwr.com/ store/product/24725854/), Accessed on January 30th 2019.

[242]. D. Sher, (Ed: https://3dprintingindustry.com/news/), Accessed on January 30th 2019.

[243]. a) Cellink, in https://cellink.com/, Accessed on January 30th 2019;b) D. Printing.com, https:// 3dprinting.com/bio-printing/), Accessed on January 30th 2019.

[244]. A. Solutions, http://www.bioassemblybot.com/, Accessed on January 30th 2019.

[245]. a) Formlabs, http://formlabs.com/, Accessed on January 30th 2019;b) Little RP, http:// www.littlerp.com/, Accessed on January 30th 2019;c) Autodesk, https://ember.autodesk.com/, Accessed on January 30th 2019.

[246]. Cyfuse, http://www.cyfusebio.com/en/regenova.html, Accessed on January 30th 2019.

[247]. Aspect Biosystems, http://aspectbiosystems.com/, Accessed on January 30th 2019.

[248]. n3D, Biosciences, http://www.n3dbio.com/products/, Accessed on January 30th 2019.

[249]. Stoltz J, Zhang L, Ye J, De Isla N, Biomed. Mater. Eng. 2017, 28, 121.

[250]. Faramarzi N, Yazdi I, Nabavinia M, Gemma A, Fanelli A, Caizzone A, Ptaszek L, Sinha I, Khademhosseini A, Ruskin J, Tamayol A, Adv. Mater. 2018, 7, 1701347.

[251]. Sheridan JD, Cell Communication and Growth, Springer, Boston, MA, 1987. 


\section{A Stereolithography Bioprinting}

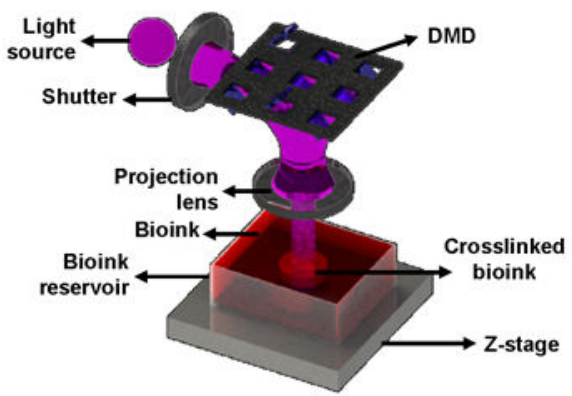

D Extrusion Bioprinting

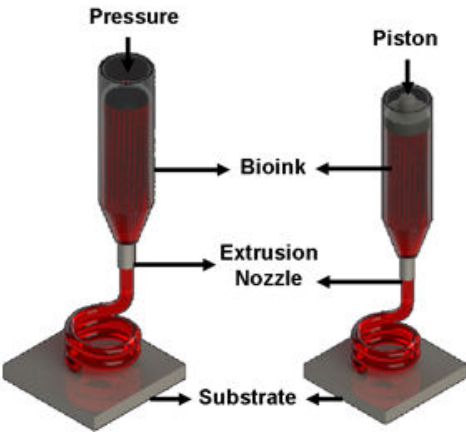

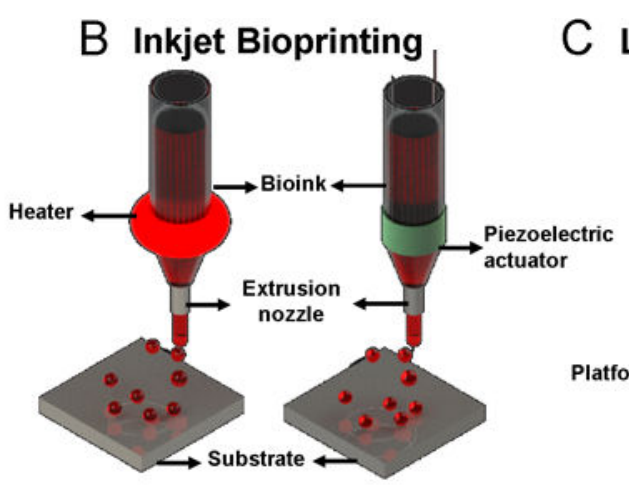

C Laser-Assisted Bioprinting

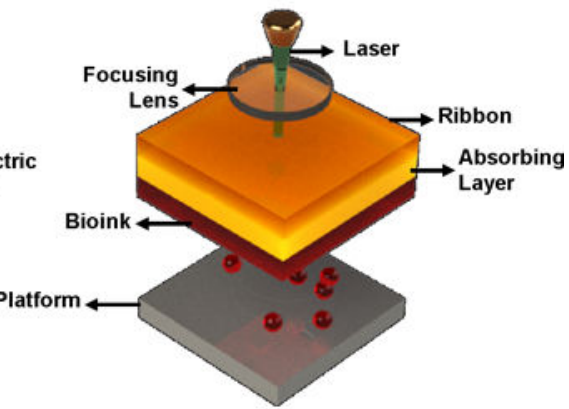

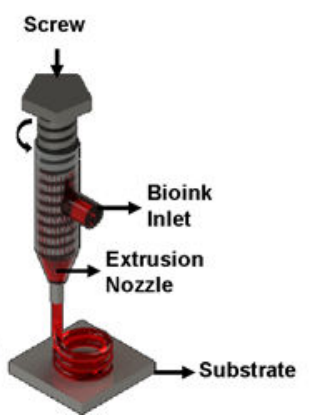
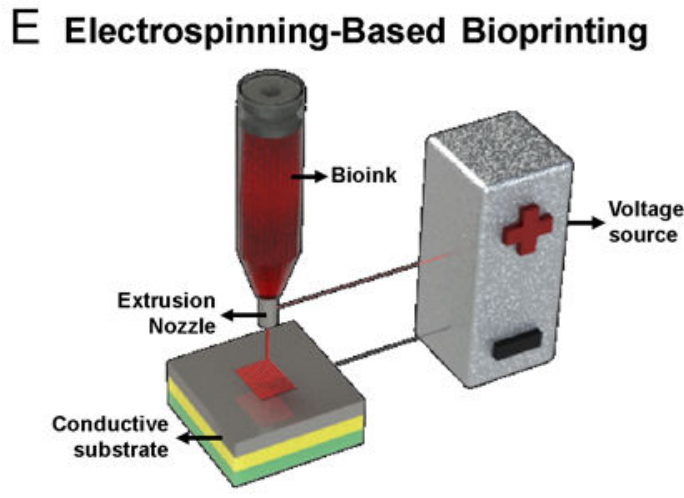

Figure 1.

Schematic representations of the different strategies currently used in 3D bioprinting. (A) Stereolithography-based bioprinting based on DMD. (B) Inkjet bioprinting using (left) thermal or (right) piezoelectric actuation. (C) Laser-assisted bioprinting. (D) Extrusionbased bioprinting strategies. From left to right: pressure-based (illustrated without valve), piston-based, and screw based. (E) EBB. 
A

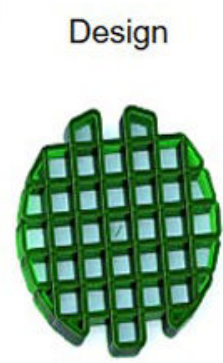

3D Bioprinting

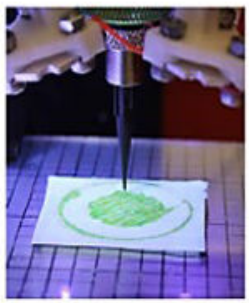

GelMA +

Nanosilicates
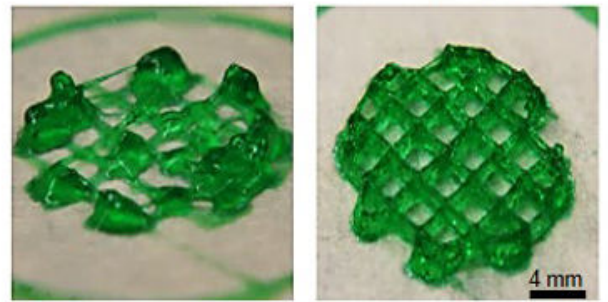

B

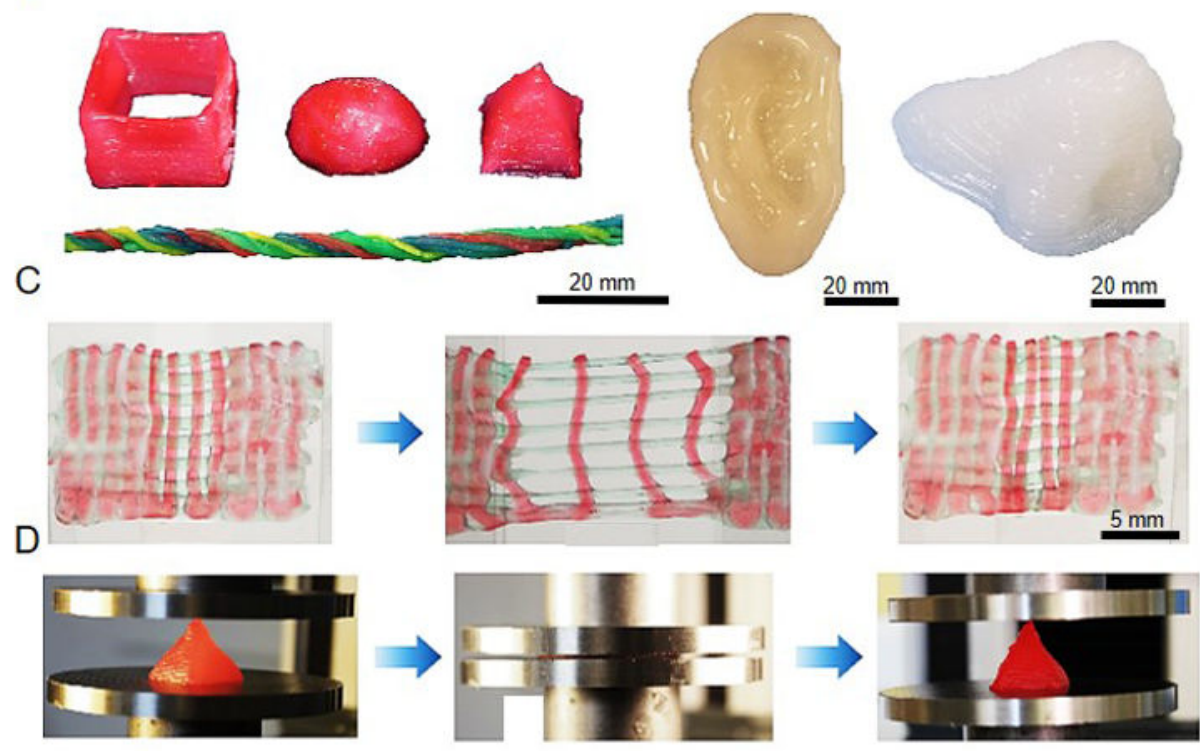

E

Design
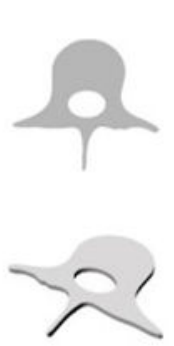

MeHA polymer in hydrogel (\%)
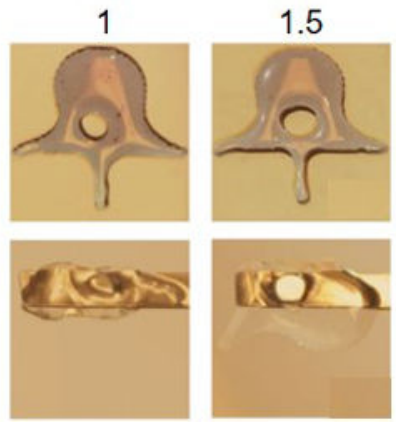

2
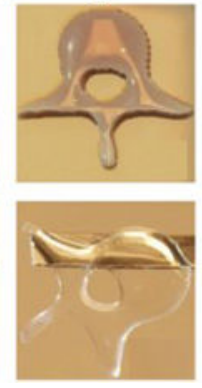

2.5
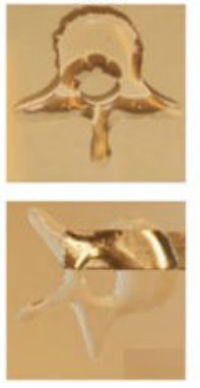

3
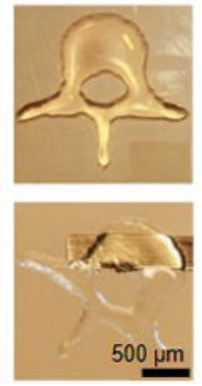

Figure 2.

Bioprinting of strong hydrogel constructs with polymer-nanoclay composite bioinks. (A) Bioprinting of GelMA enriched with nanosilicate to achieve rigid complex structures.

Reproduced with permission from ref. ${ }^{[70]}$. (B) Various 3D constructs bioprinted with a PEGalginate-nanoclay hydrogel (from left to right: hollow cube, hemisphere, pyramid, twisted bundle, ear shape and nose shape). (C) Bioprinted PEG-alginate-nanoclay mesh undergoing repeated uniaxial stretching and recovering to its original shape. (D) Bioprinted PEGalginate-nanoclay pyramid undergoing repeated compressions and recovering to its original 
shape. Reproduced with permission from ref. ${ }^{[81]}$. (E) Human L3 vertebrae bioprinted with various concentrations of MeHA in the bioink. Reproduced with permission from ref. ${ }^{[83]}$. 
A

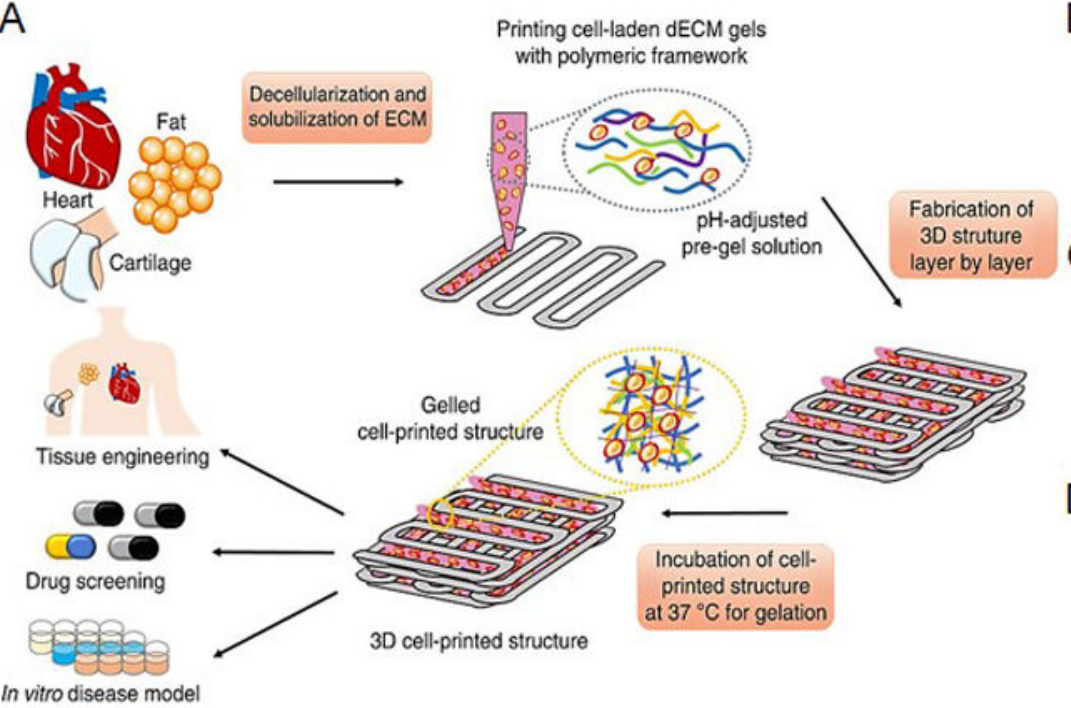

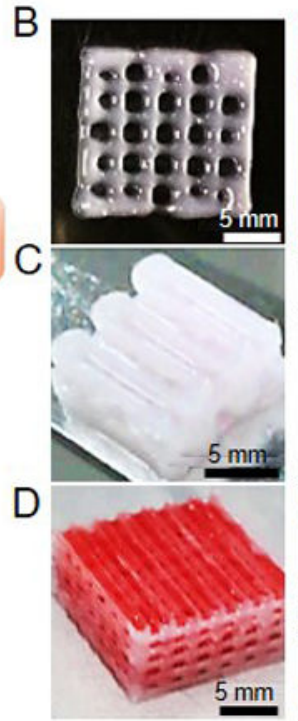

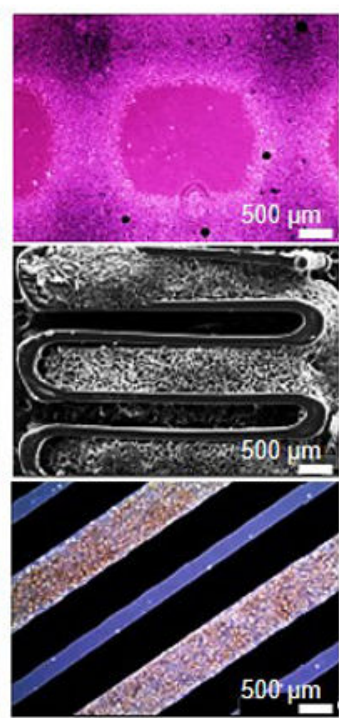

E

$\mathrm{F}$ pattern $/ \mathrm{M}$ patch
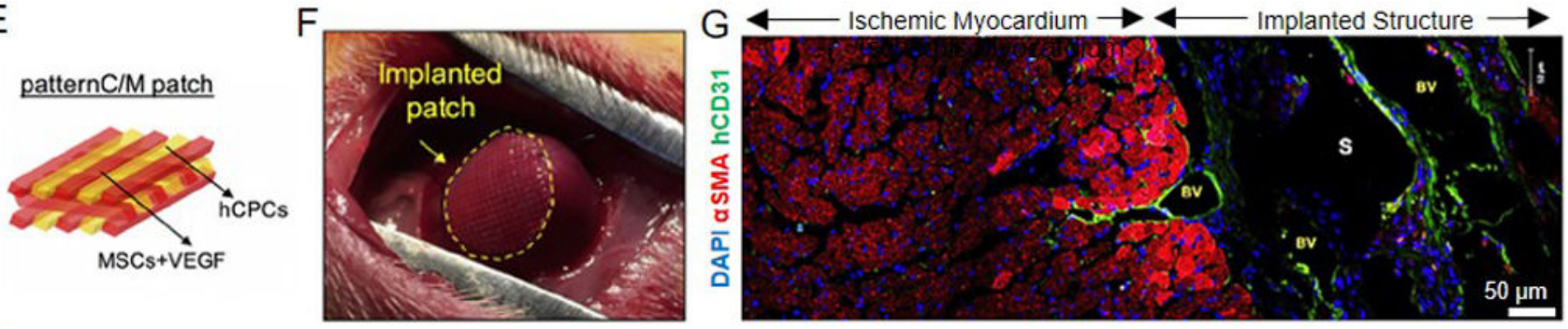

$\mathrm{H} \quad$ Native

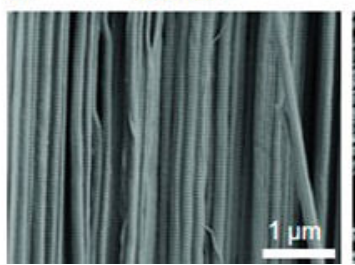

dtECM
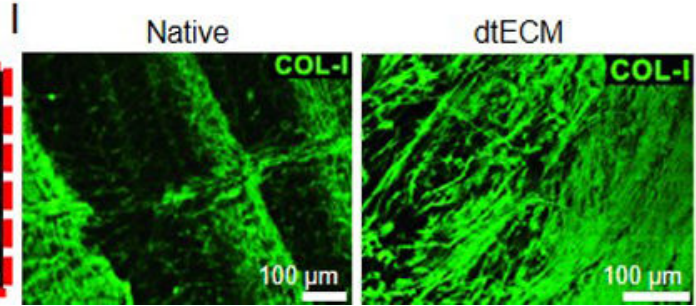

Figure 3.

Bioprinting of tissue constructs with tissue-specific dECM bioinks. (A) Schematic representation of the bioprinting process using dECM bioinks. (B-D) Multi-layered tissue constructs were bioprinted with (B) cardiac-specific dECM bioink and representative microscopic image, (C) cartilage-specific dECM bioink and representative SEM image, and (D) adipose-specific dECM bioink and representative microscopic image. Reproduced with permission from ref. ${ }^{[63]}$. (E) Schematic representation of a bioprinted pre-vascularized stem cell patch using dECM bioink and supporting PCL. (F) Photograph of implanted cardiac dECM/PCL patch. (G) Cardiomyogenesis and neovascularization of the pre-vascularized stem cell patch. Reproduced with permission from ref. ${ }^{[92]}$. (H) SEM images of native porcine Achilles tendon and dECM obtained from the same tissue. (I) Collagen type I immunostaining of native and $\mathrm{dECM}$ tendon tissues. Reproduced with permission from ref. [93]. 
A

B
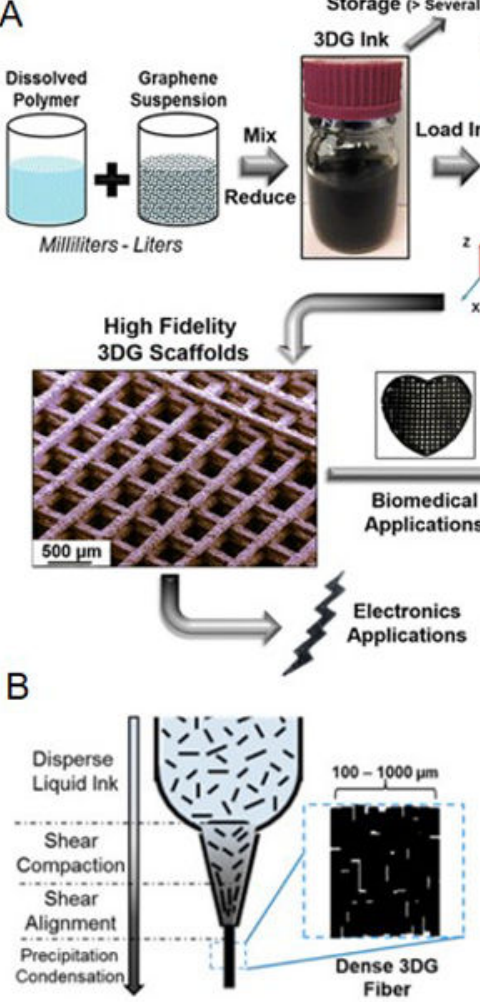

Milliliters - Liters

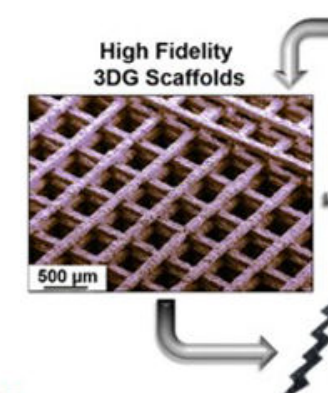

Storage (> Several Months)

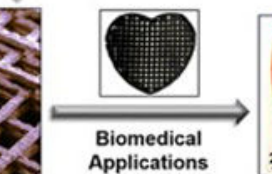

Figure 4.

Bioprinting of tissue constructs using bioinks with high conductivity and favorable bioelectronics properties. (A) Schematic showing the preparation of PLG-graphene conductive bioink and fabrication of tissue constructs by 3D printing. (B) (Left) Schematic of the extrusion of a bioink containing $60 \%$ grapheme and $40 \%$ PLG displaying how shear forces at the nozzle tip could result in graphene flake alignment. (Right) SEM images of the printed partially aligned grapheme flakes. Reproduced with permission from ref. ${ }^{[105]}$. (C) Schematic diagram of the bioprinting process for 3D electrically conductive constructs using DNA/HA-coated CNT bionks. (D) Cast GelMA constructs containing 3D-bioprinted CNT microfibers. (E) Immunostaining for sarcomeric a-actinin (green), nuclei (blue), and Cx43 (red) of cardiomyocytes encapsulated in the GelMA hydrogels containing bioprinted CNT microfibers 10 days post-bioprinting. Reproduced with permission from ref. ${ }^{[113]}$. (F) A 3Dprinted wearable sensor for finger gesture recognition. Reproduced with permission from ref. ${ }^{[116]}$

Small. Author manuscript; available in PMC 2020 June 01. 
A

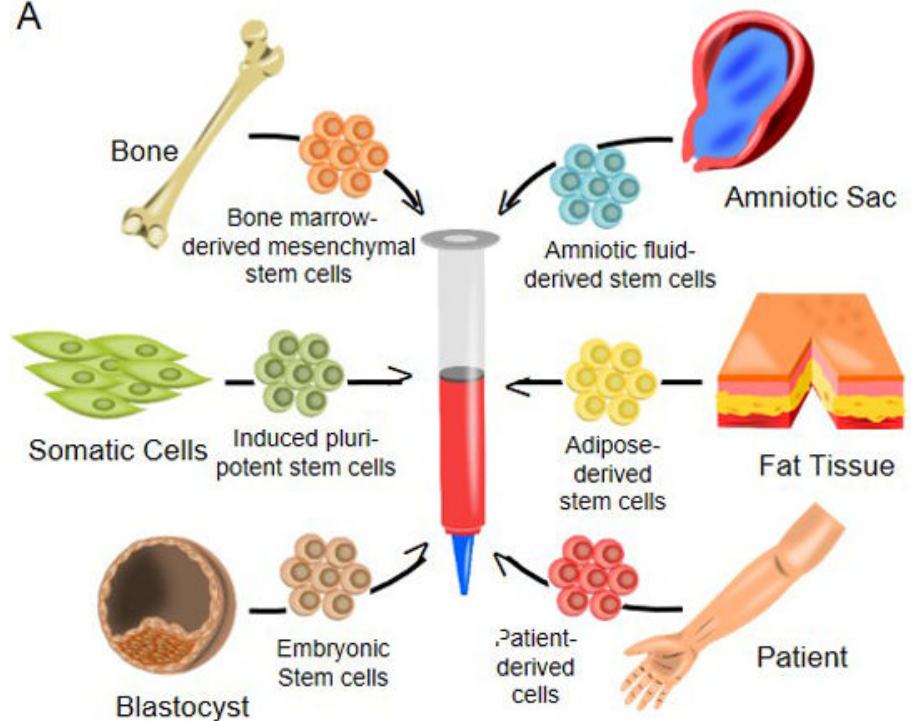

B

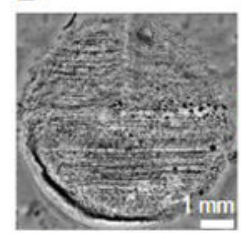

$\mathrm{E}$

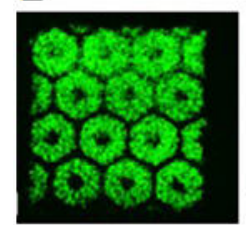

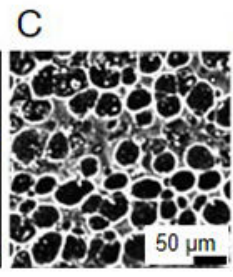

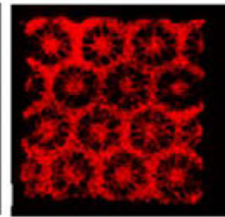

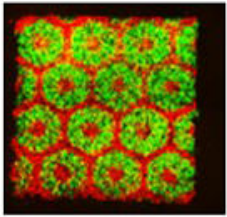

D

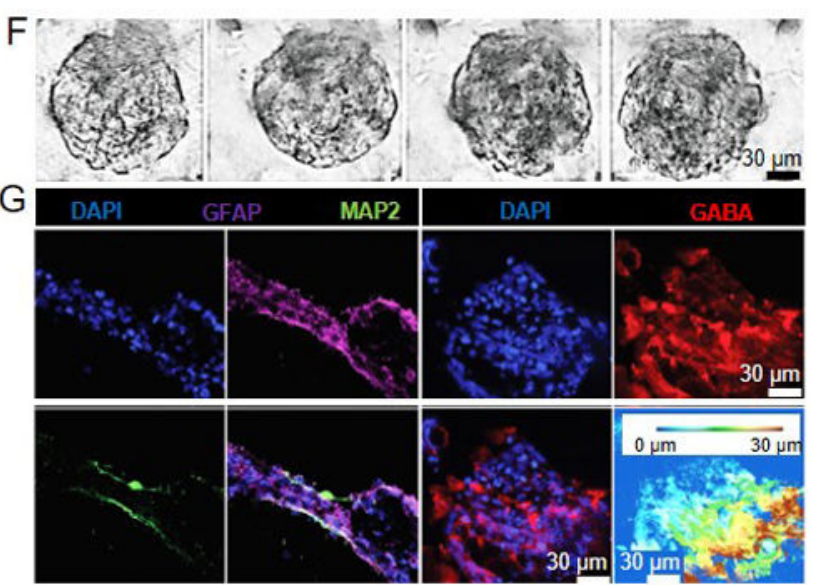

$\mathrm{H}$
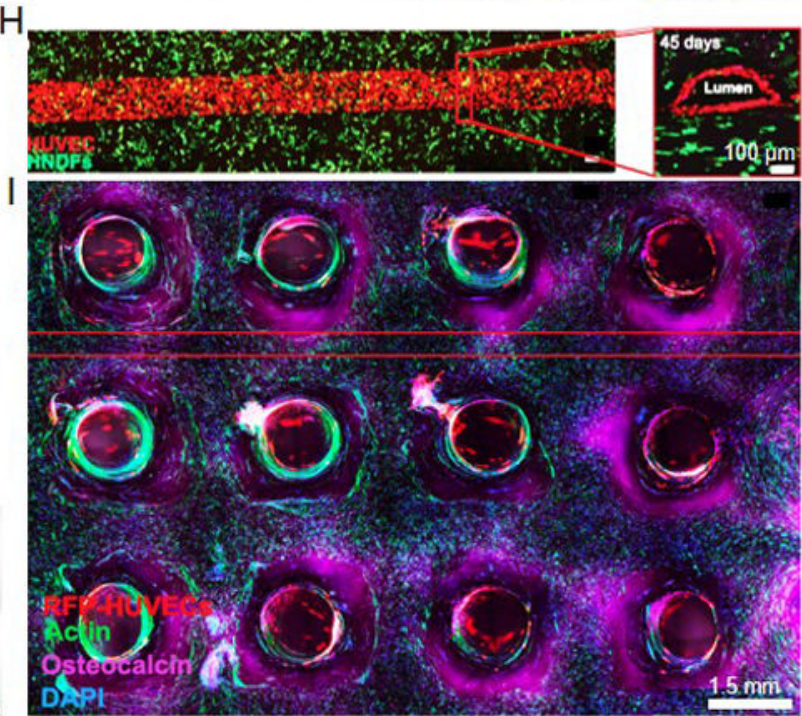

Figure 5.

Cell sources for bioprinting. (A) Schematic overview of most common cell types used for bioprinting applications. (B) Bright-field and fluorescence images of multi-cellular constructs fabricated using inkjet bioprinting containing BAECs (green-labeled), human AFSCs (blue-labeled), and canine SMCs (red-labeled). (C) Microscopic image of microstructure of the multi-cellular construct. (D) Gross view of the multi-cellular construct 2 weeks after implantation in a mouse model. Reproduced with permission from ref. ${ }^{[128]}$. (E) Fluorescence and bright-field images of labeled iPSC-derived hepatocytes in green and a mixture of HUVECs and ASCs in red forming characteristic hepatic hexagon structures bioprinted by stereolithography. Reproduced with permission from ref. ${ }^{[135]}$. (F) Selforganized embryoids from iPSCs bioprinted in a supporting hydrogel matrix consisting of alginate, chitosan, and agarose (from left to right: $0 \mathrm{~min}, 350 \mathrm{~min}, 890 \mathrm{~min}$ and $1290 \mathrm{~min}$. (G) Immunofluorescence staining in 3D-bioprinted embryoids of MAP2, GFAP, and GABA expressions as well as depth coding along z-axis from 0-47 $\mu \mathrm{m}$. Reproduced with permission from ref. ${ }^{[136]}$. (H) Confocal images of a bioprinted blood vessel after long-term perfusion in a thick tissue construct. HUVECs appear in red and HNDFs in green. (I) 
Confocal microscope image of a $1 \mathrm{~cm}$-thick vascularized osteogenic tissue construct containing HUVECs, HNDFs, and hMSCs. Reproduced with permission from ref. ${ }^{[140]}$. 
A

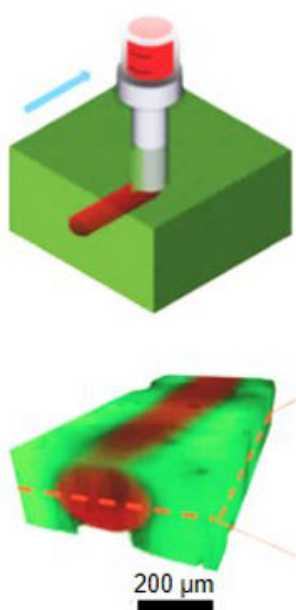

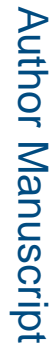

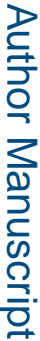

F

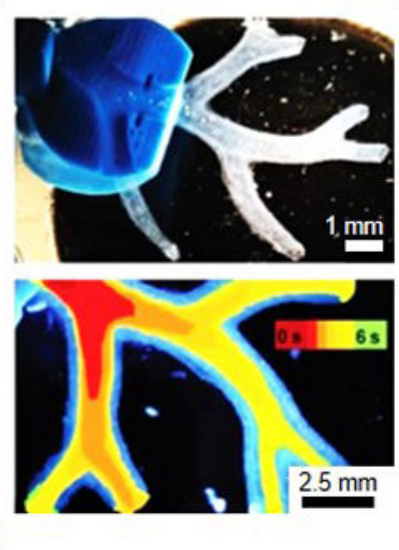

$\mathrm{J}$

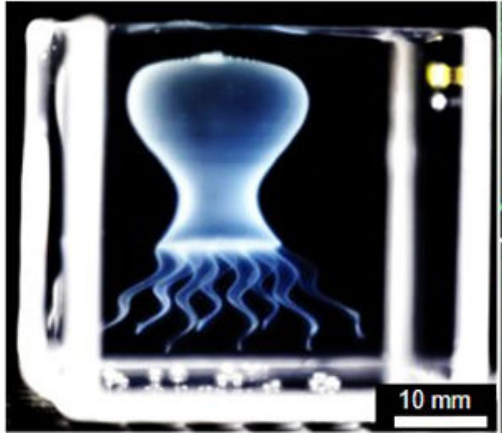

B
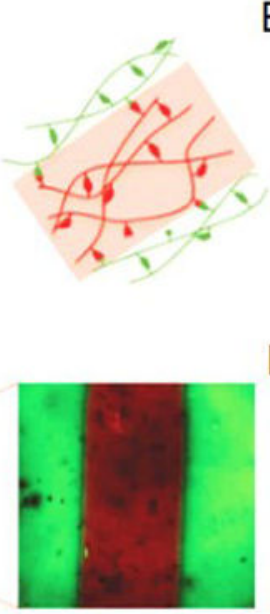

G

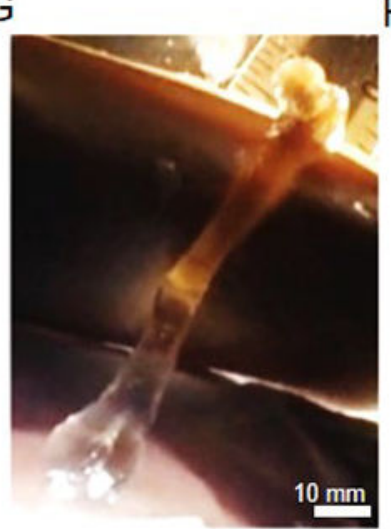

$\mathrm{H}$

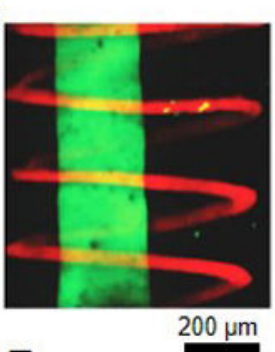

E

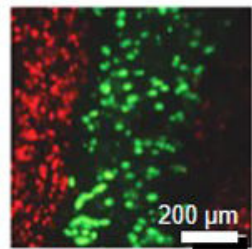

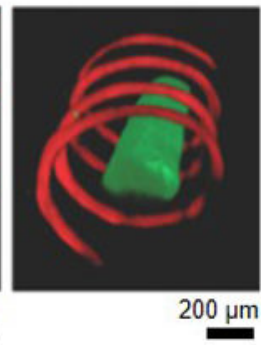

Red: 3T3

Green: MSC

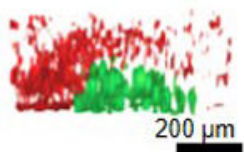

$100 \mu \mathrm{m}$

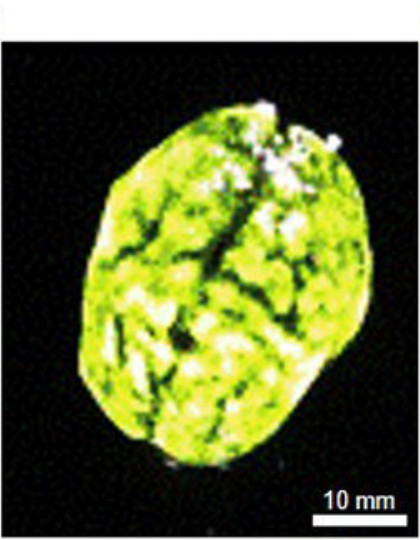

K
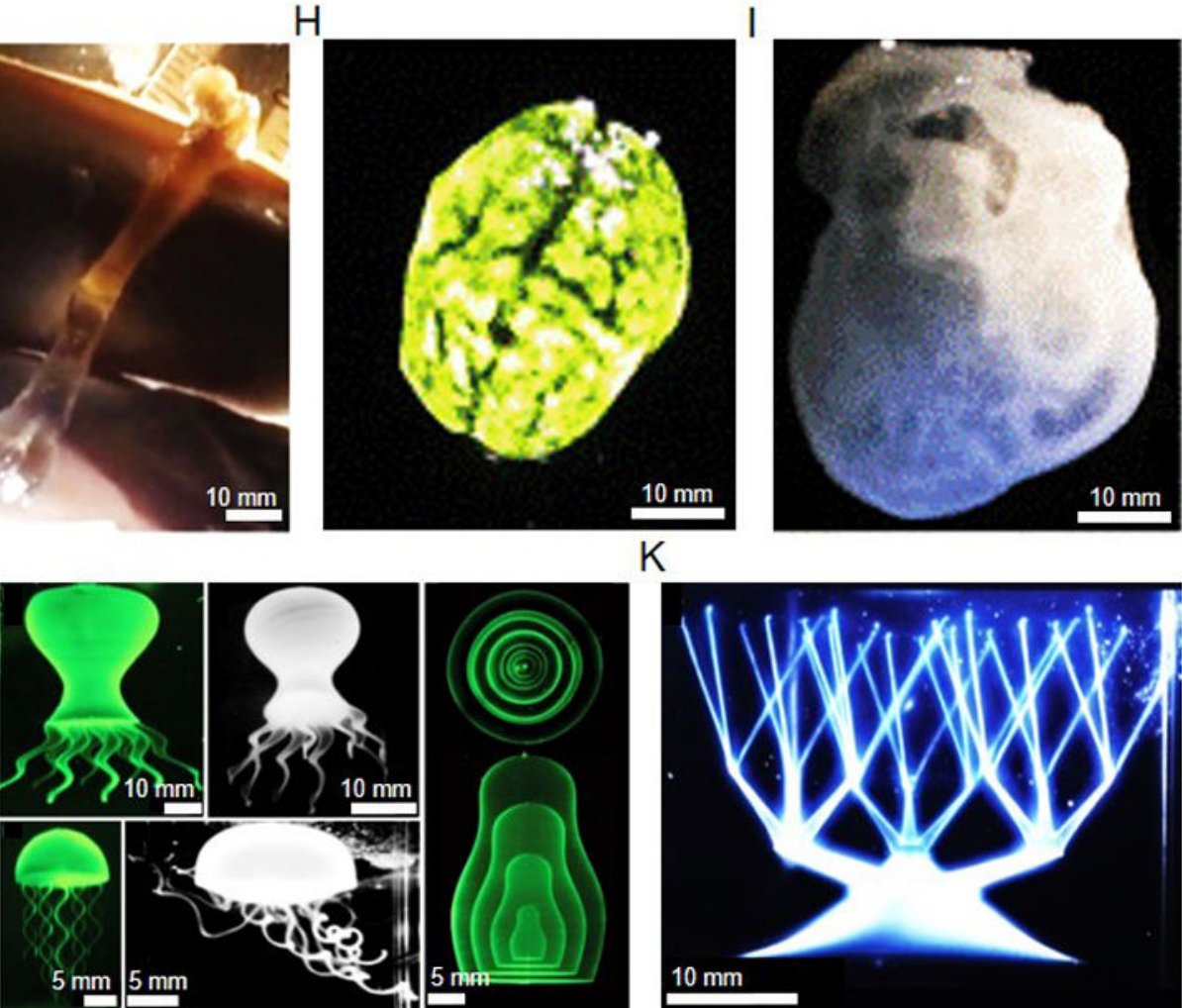

Figure 6.

Freeform embedded bioprinting of different shapes and constructs. (A) Schematic showing extrusion of a supramolecular HA-Ad guest bioink into a HA-CD host matrix. (B)

Rhodamine-labeled filaments extruded into a fluorescein-labeled support hydrogel from using different needle gauges. (C) Confocal images of a filament of a fluorescein-labeled bioink and then a continuous spiral of a second, rhodamine-labeled bioink extruded into an unlabeled support hydrogel. (D) Confocal images of discrete pockets of a rhodamine-labeled bioink extruded into an unlabeled support hydrogel. (E) Confocal images of mesenchymal stem cells (green) printed within a bioink into a support hydrogel containing NIH/3T3 
fibroblasts (red). Reproduced with ref. ${ }^{[144]}$. (F) Model and bioprinted structure of a human right coronary arterial based on a 3D MRI image with perfusable structure. (G) Model of a human femur and printed design with alginate bioink after removal from the support hydrogel. (H, I) Bioprinted scaffolds with complex internal and external architectures based on 3D imaging data from whole organs for $(\mathrm{H})$ brain and (I) heart. Reproduced with permission from ref. ${ }^{[145]}$. (J) Thin-shell octopus bioprinted in different designs and a model of a Russian doll with several layers bioprinted in the support hydrogel. (K) A continuous network of hollow vessels with features spanning several orders of magnitude in diameter and aspect ratio. Reproduced with permission from ref. ${ }^{[146]}$. 
A

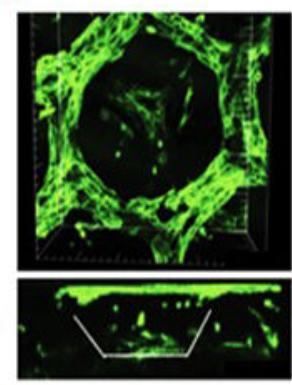

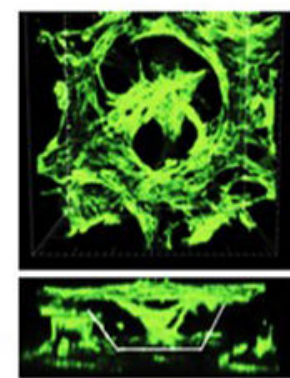

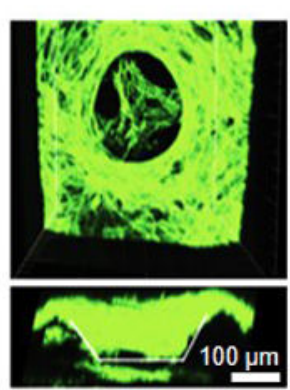

B
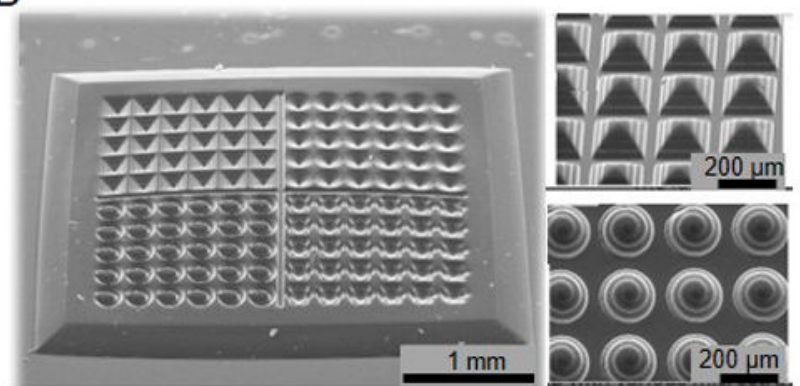

C

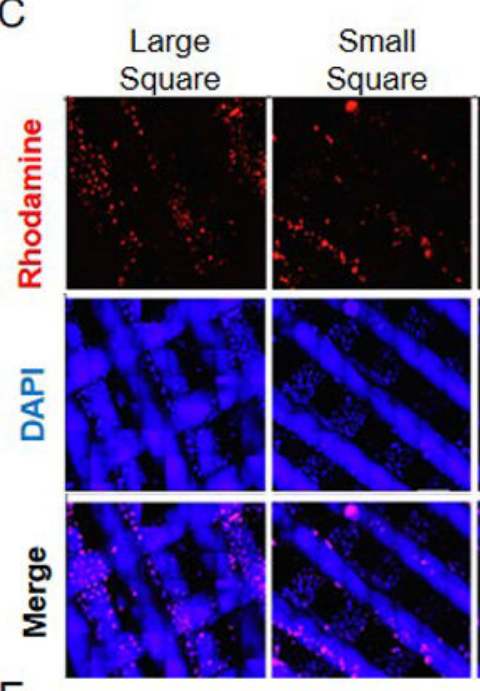

E

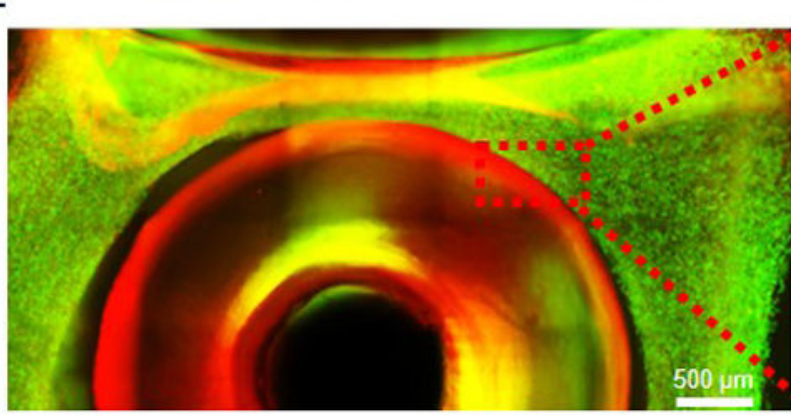

D

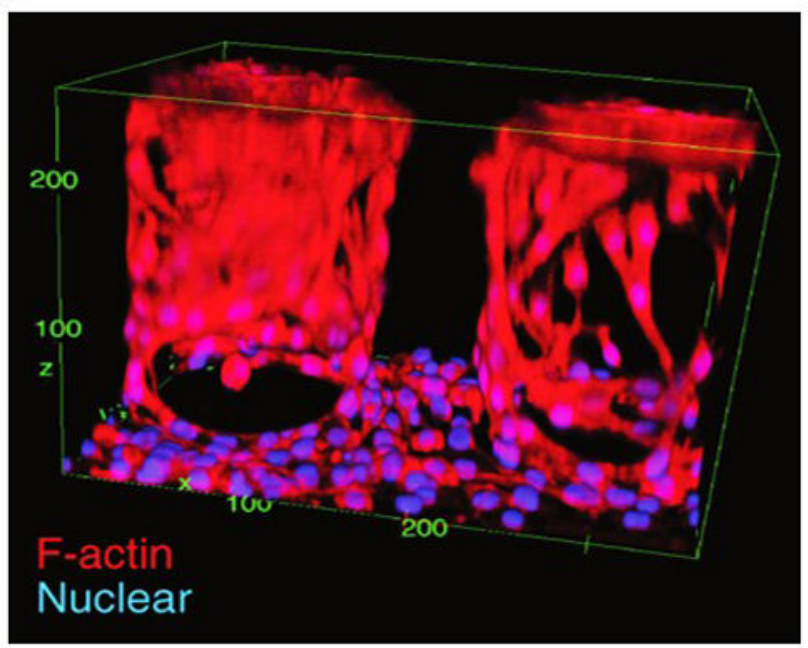

Figure 7.

Stereolithography-based bioprinting. (A) Confocal images of HUVECs spreading on bioprinted scaffolds. Reproduced with permission from ref. ${ }^{[148]}$. (B) SEM images of PEGDA microwells of different shapes. Reproduced with permission from ref. ${ }^{[149]}$. (C) Confocal images of MDA-MB-231 cells on 3D-bioprinted bone matrices of different shapes, stained for phalloidin (red) and nuclei (blue). Reproduced with permission from ref. ${ }^{[150]}$. (D) Confocal fluorescence image of encapsulated NIH/3T3 fibroblasts in a bioprinted GelMA scaffold showing cell spreading and active cell-material interactions. Reproduced with permission from ref. ${ }^{[151]}$. (E) Bioprinted hybrid construct with HUVECs incorporated into a collagen hydrogel surrounded by a PEGDA scaffold. Reproduced with permission from ref. [152]. 

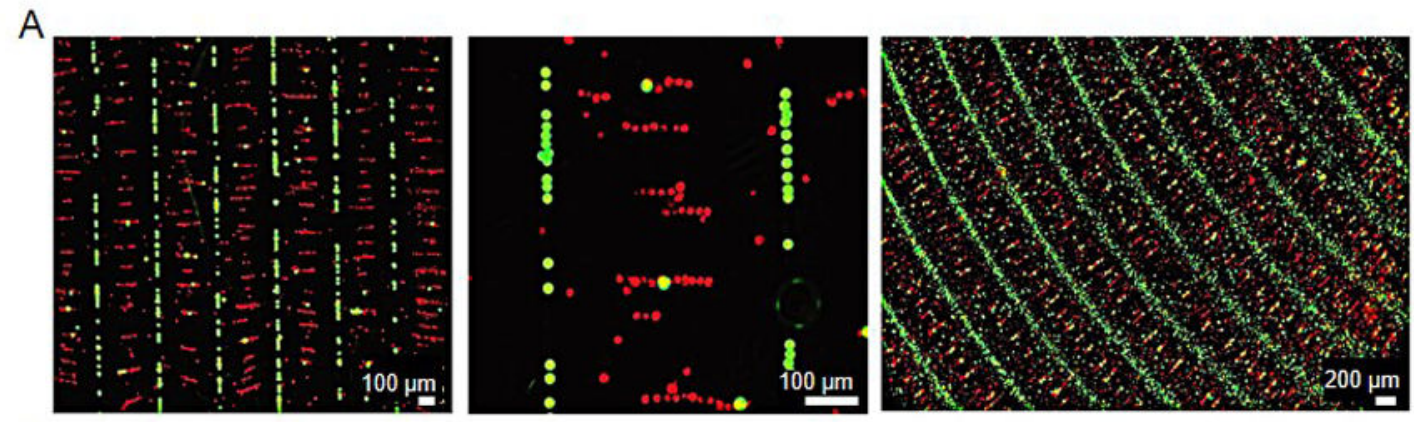

B
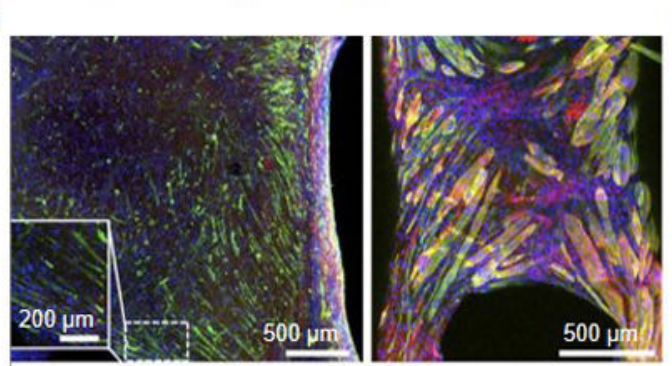

C
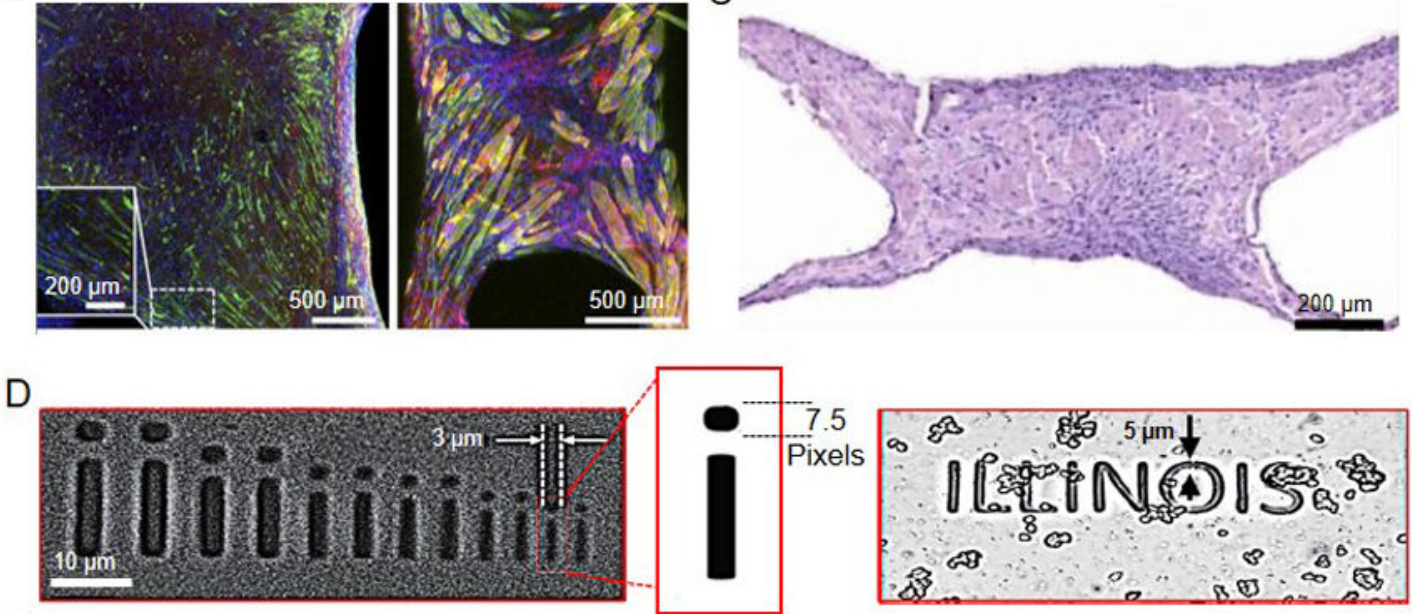

$\mathrm{E}$
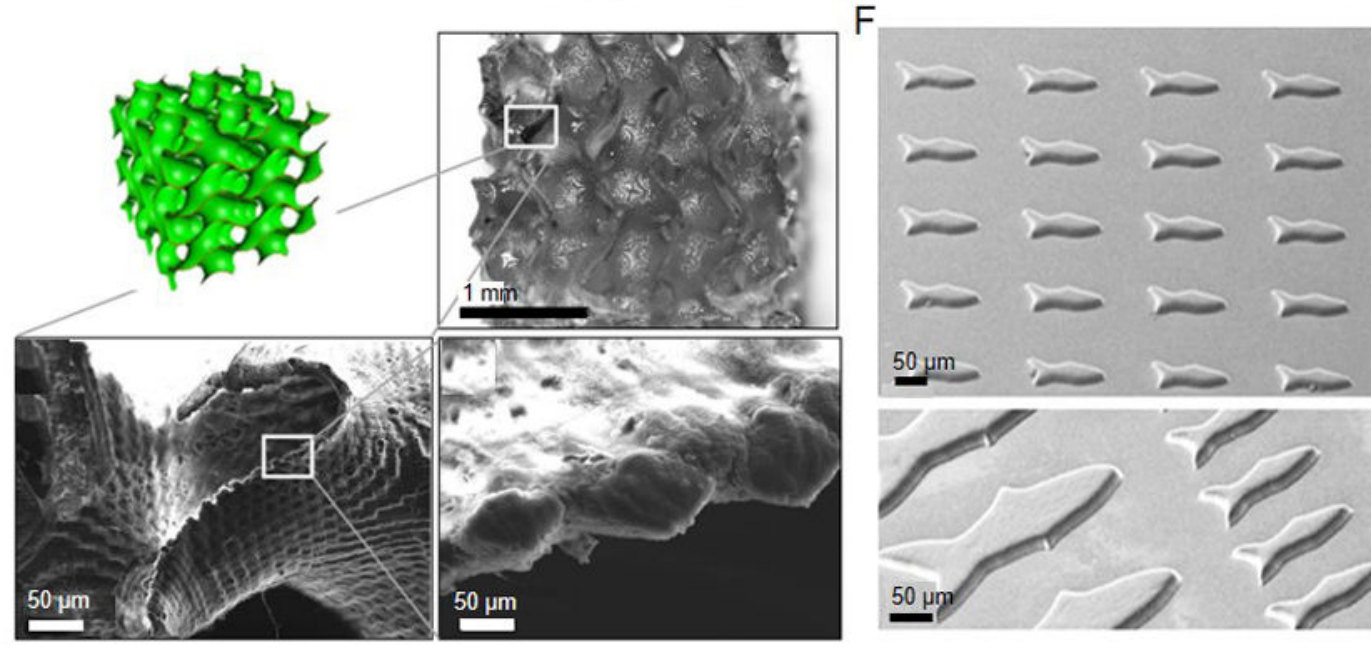

Figure 8.

Recent advances in stereolithography-based bioprinting. (A) Patterning of $\mathrm{C} 2 \mathrm{C} 12$ cells (red) and polystyrene beads (green) in a PEGDA hydrogel based on a combination of dielectrophoresis and stereolithography to demonstrate the large scale patterns of mammalian cells in 3D hydrogels. Reproduced with permission from ref. ${ }^{[153]}$. (B) Characterization of muscle strip containing $\mathrm{C} 2 \mathrm{C} 12$ skeletal muscle fibroblasts with $\mathrm{ECM}$ proteins fibrin (left) or collagen type I (right) attached to 3D-printed holders to evaluate mechanical properties of the bioprinted muscle. (C) H\&E staining of muscle strips 
containing C2C12 and fibrin. Reproduced with permission from ref. ${ }^{[154]}$. (D) Highresolution production of different structures using micro-stereolithography in PEGDMA hydrogel. Reproduced with permission from ref. ${ }^{[28]}$. (E) Gyroid construct using a blend bioink of PVA-MA and GelMA and specially designed photoinitiators with (top) respective 3D CAD model and photograph as well as (bottom) SEM images. Reproduced with permission from ref. ${ }^{[155]}$. (F) SEM image of stereolithographically bioprinted microfish, which exhibited chemically powered and magnetically guided propulsion as well as efficient detoxification capabilities. Reproduced with permission from ref. ${ }^{[157]}$. 


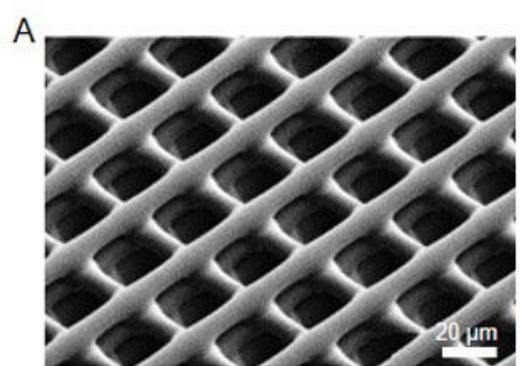

B
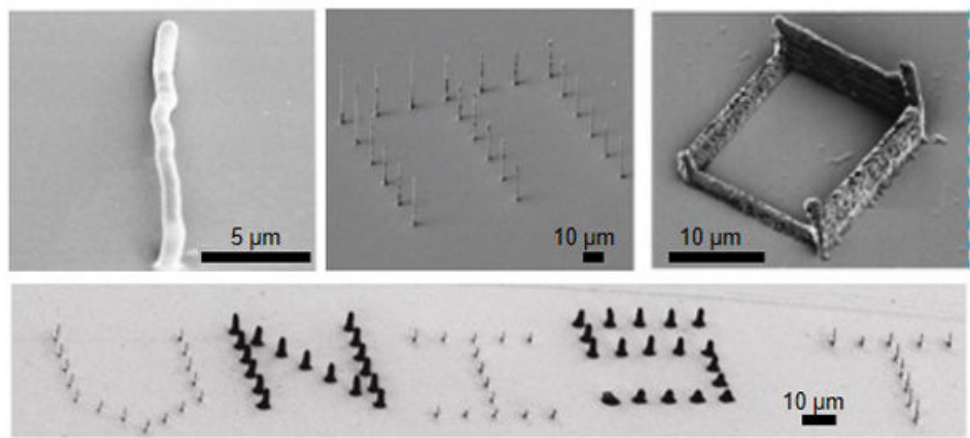

$\mathrm{H}$
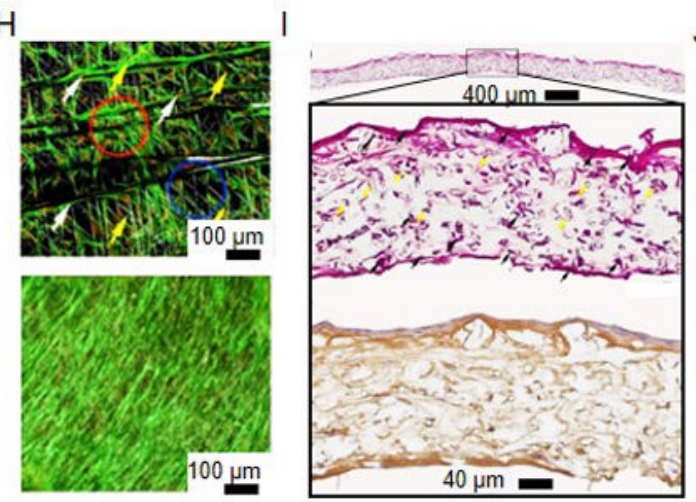

J

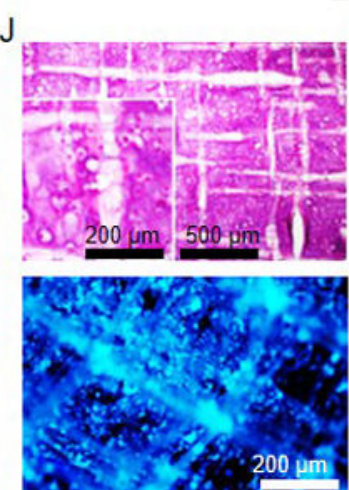

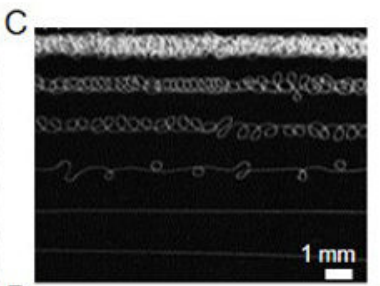

D

$1 \mathrm{~mm}$

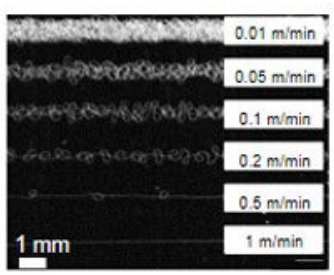

E

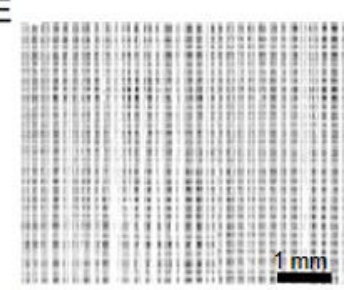

F
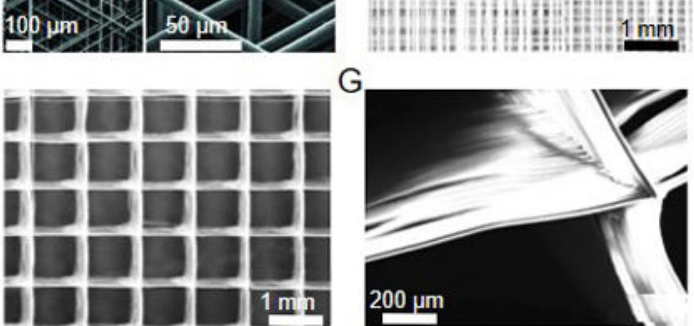

K

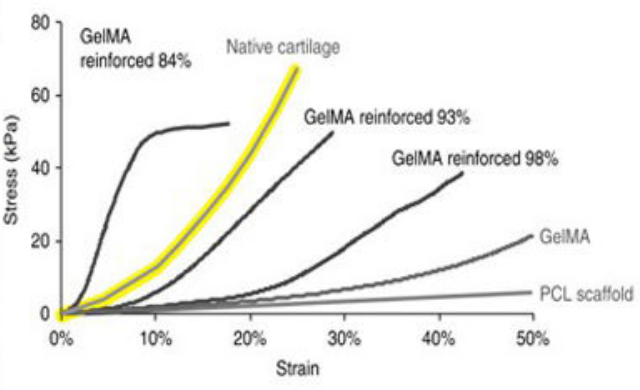

Figure 9.

Bioprinted constructs in various architectures at high resolution. (A) SEM micrographs of 3D pHEMA scaffolds of varying architecture produced by extrusion-based bioprinting. Reproduced with permission from ref.[161]. (B) Enlarged SEM photomicrograph showing various nanofibers based on EBB with a diameter of $700 \mathrm{~nm}$ (from left to right: Single nanofiber, letter "E"-shaped array of anthracene pillars, Rectangular anthracene structure composed of four flat walls and (bottom) "UNIST"-shaped array of $\mathrm{Cu}$ and anthracene pillars. Black and white pillars in the array are composed of anthracene and $\mathrm{Cu}$, respectively. Reproduced with permission from ref. ${ }^{[48]}$. (C) Photograph of lines of PCL fibers melt electrospun at different extrusion speeds. (D) PCL scaffolds assembled with fibrous layers oriented at $60^{\circ}$. (E, F) Thin PCL fibers stacked in a $0-90^{\circ}$ with spacing of (E) 0.2 and (F) 1.0 $\mathrm{mm}$ as visualized with stereomicroscopy. (G) Detailed image of printed PCL fibers that fused at the cross-sections. Reproduced with permission from refs. ${ }^{[51,80]}$. (H) Confocal microscopy of fibroblasts on an EBB-bioprinted scaffold after (top) 7 and (bottom) 14 days. The white arrows show fibroblast migration along the fibers and the yellow arrows show spreading across scaffold voids. (I) (Top) H\&E staining and (bottom) collagen type I 
staining of the bioprinted PCL scaffold. Reproduced with permission from ref. ${ }^{[51]}$. (J) (Top) H\&E staining and (bottom) DAPI staining of chondrocytes bioprinted in an EBB-produced scaffold aimed to regenerate cartilage tissue. Reproduced with permission from ref. ${ }^{[80]}$. (K) Stress-strain curves of the PCL scaffold, GelMA, and reinforced GelMA, approaching the curve of native cartilage (yellow). Reproduced with permission from ref. ${ }^{[167]}$. 
A

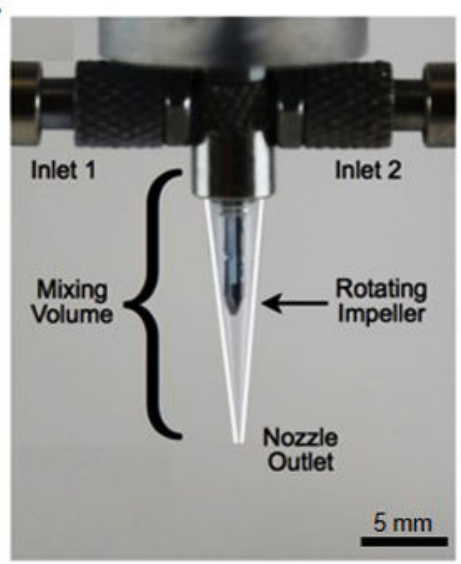

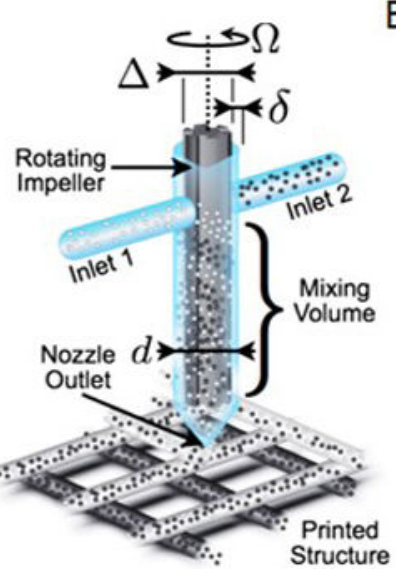

B

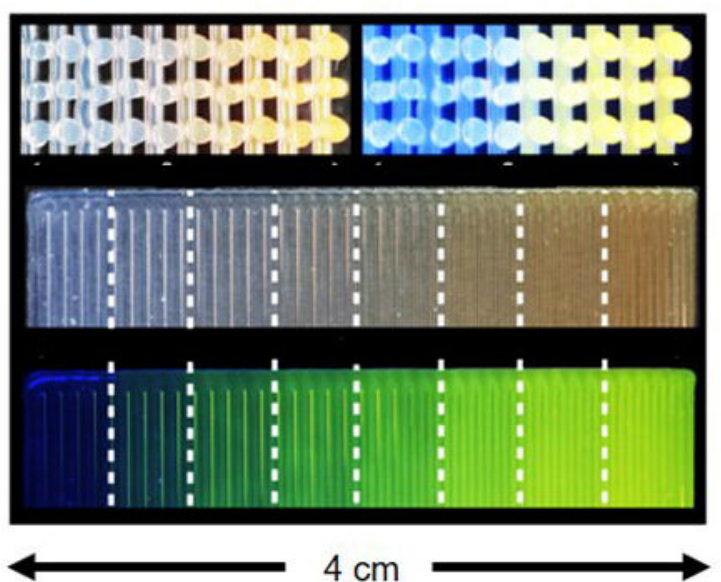

$\mathrm{C}$

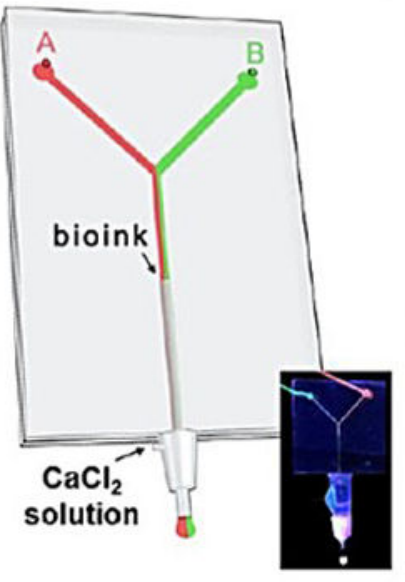

D
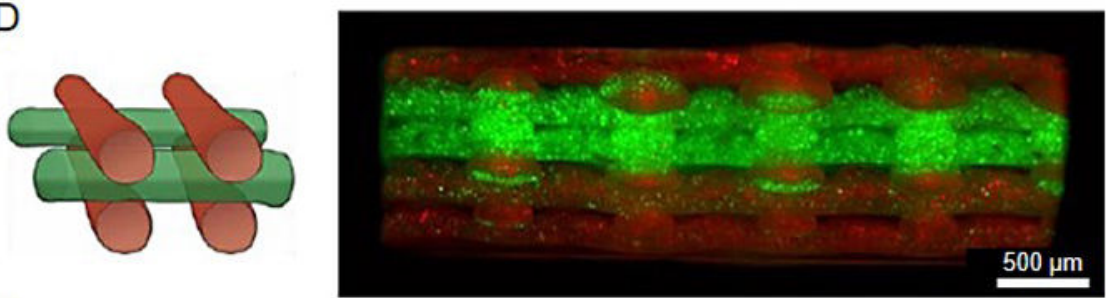

E
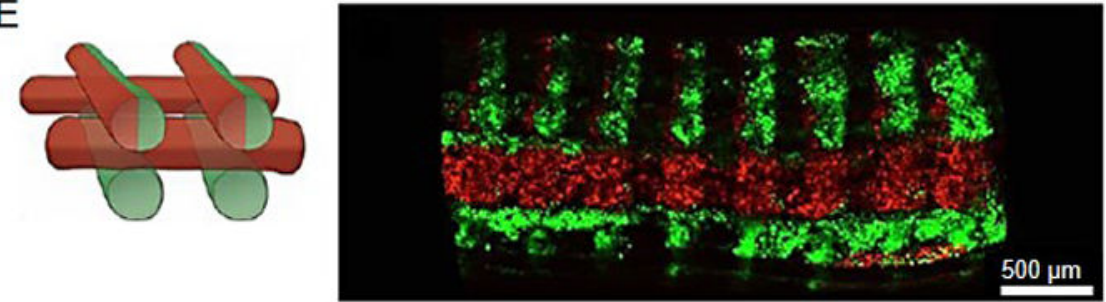

$\mathrm{F}$
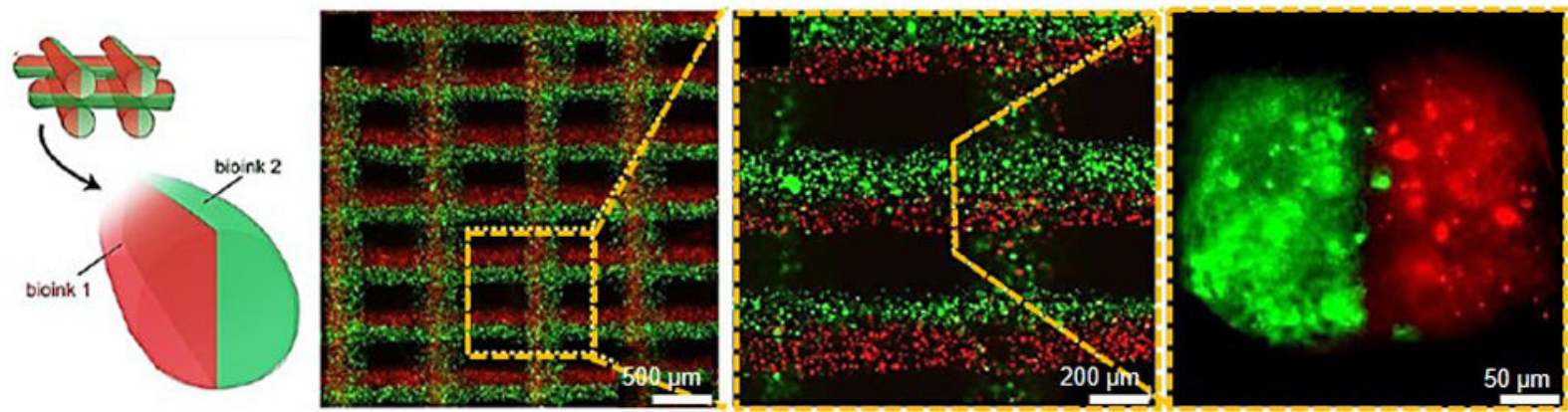

Figure 10.

Multi-material bioprinting of cell-laden tissue constructs. (A) Optimal image and schematic of an impeller-based active mixer printhead. (B) (Top) Images of the cross-section of a 3D rectangular lattice structure of showing continuous change in fluorescent pigment concentration under (left) bright light and (right) UV radiation. (Bottom) Images of a 2D carpet structure showing a discretely varying fluorescent gradient at eight different mixing ratios under (top) bright light and (bottom) UV radiation. Reproduced with permission from ref. ${ }^{[173]}$. (C) Schematic of a microfluidic system to separate two bioinks while extrusion 
trough a single nozzle. (D-F) Schematics and fluorescence microscopy images of crosssection views of 3D construct with (D) alternative deposition, (E) alternative/simultaneous deposition, and (F) simultaneous deposition. Reproduced with permission from ref. ${ }^{[69]}$. 
A

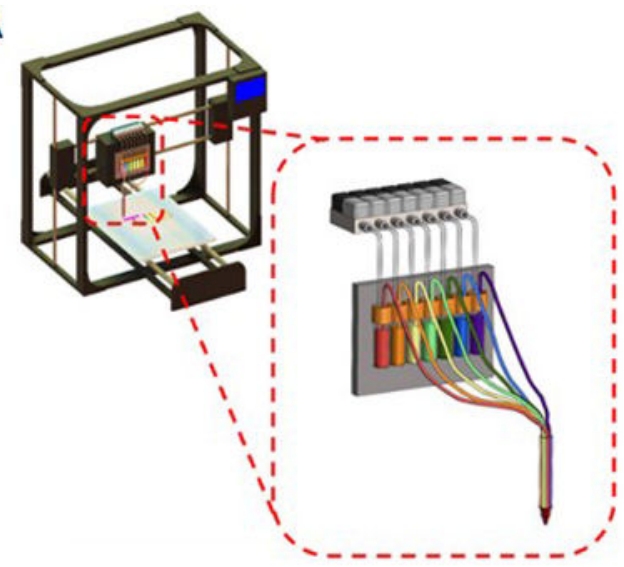

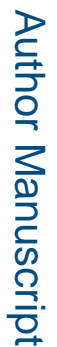

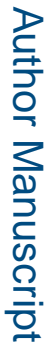

B

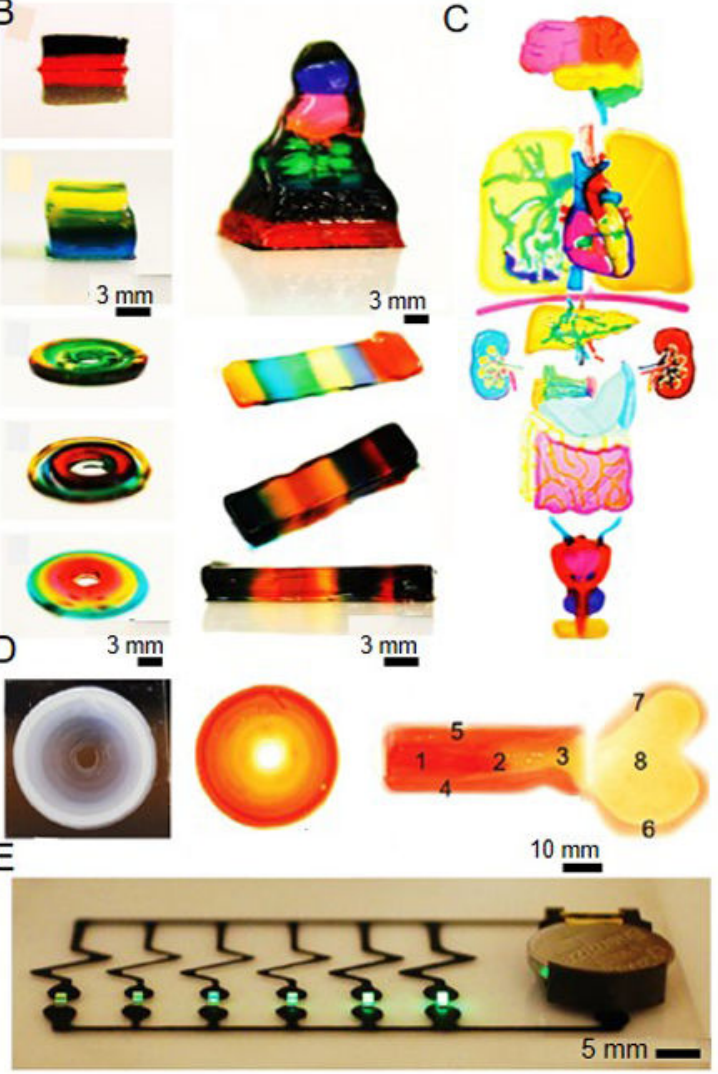

$\mathrm{F}$

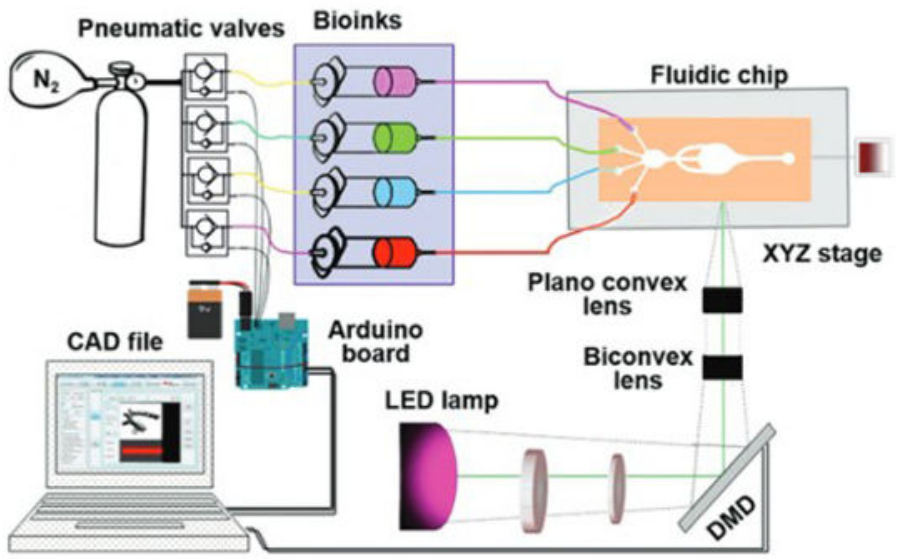

G

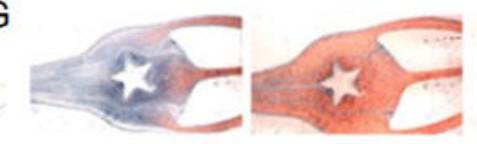

$\mathrm{H}$
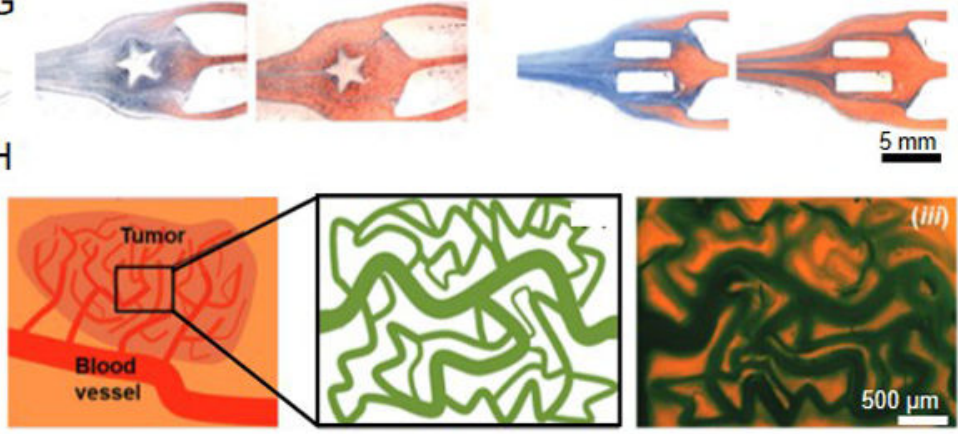

।

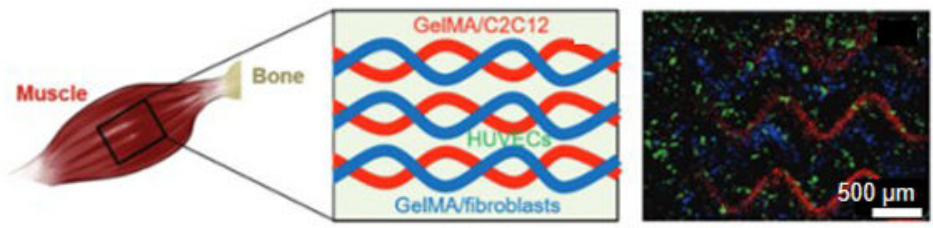

$J$

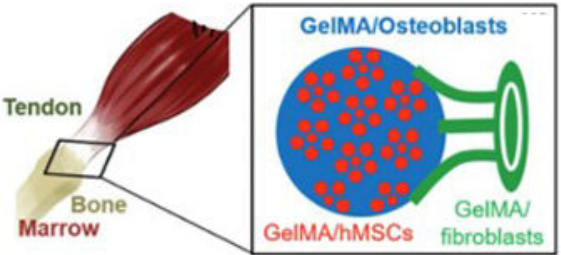

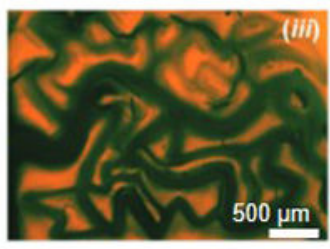

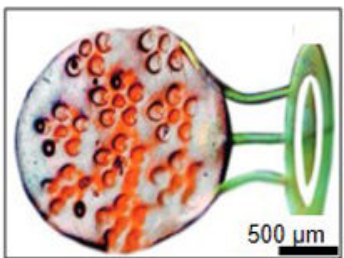

Figure 11.

Recent strategies in multi-material extrusion and stereolithographic bioprinting. (A)

Schematic of a multi-material extrusion bioprinter consisted of seven bioink outlets and a single nozzle. (B, C) Photographs of bioprinted structures with (B) cubical structures, circular structures, pyramid and stripes as well as (C) different organ designs. (D) Bioprinted GelMA-hydroxyapatite structures to display a gradient of osteogenic induction properties.

(E) Bioprinted circuit structure using different concentrations of CNTs in GelMA to create different resistance in the circuit (displayed by green LED intensity). Reproduced with permission from ref. ${ }^{[174]}$. (F) Planar schematics showing the setup of multi-material stereolithography, including a UV lamp $(385 \mathrm{~nm})$, optical lenses and objectives, a DMD 
chip, and a microfluidic device. (G) Photographs showing the bioink exchange processes in the microfluidic chip for a star pattern and two rectangular patterns. (H) A tumor angiogenesis model with (left) schematic, (middle) mask for bioprinting, and (right) the bioprinted GelMA vascularized construct perfused with food dye. (I) A skeletal muscle model with (left) schematic, (middle) mask for bioprinting, and (right) fluorescence micrograph of the bioprinted GelMA construct containing patterned $\mathrm{C} 2 \mathrm{C} 12$ cells (red) and fibroblasts (blue). (J) A tendon-to-bone insertion model with (left) schematic, (middle) mask for bioprinting, and (right) bright-field optical image showing the bioprinted dye-laden GelMA construct containing the corresponding features. Reproduced with permission from ref. ${ }^{[180]}$. 
A

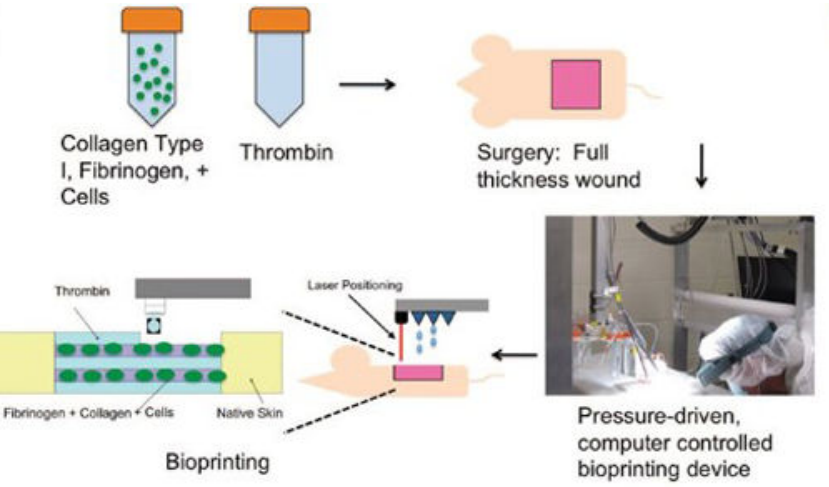

B
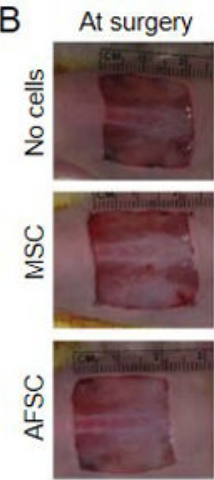
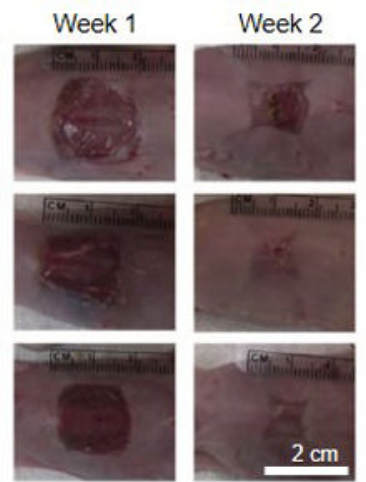

C
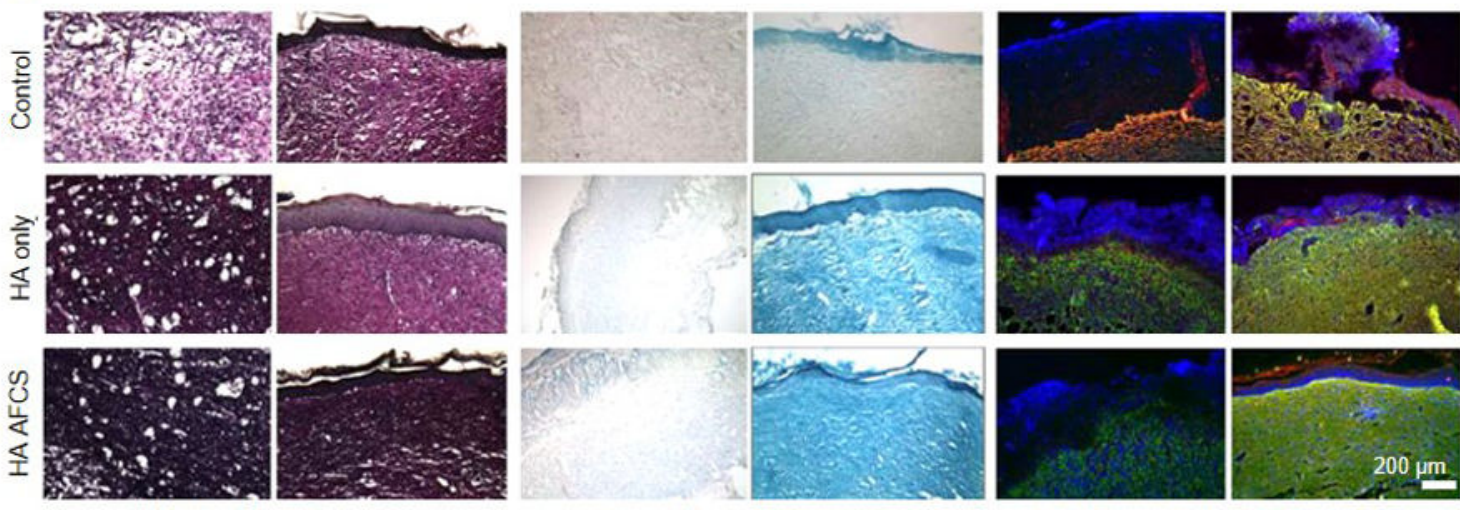

Verhoeff-Van Geison (elastin black)

D E (polysaccharides/GAGs blue)
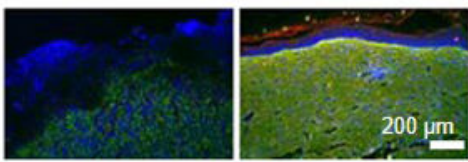

Immunohistochemistry (collagen I red, III green)
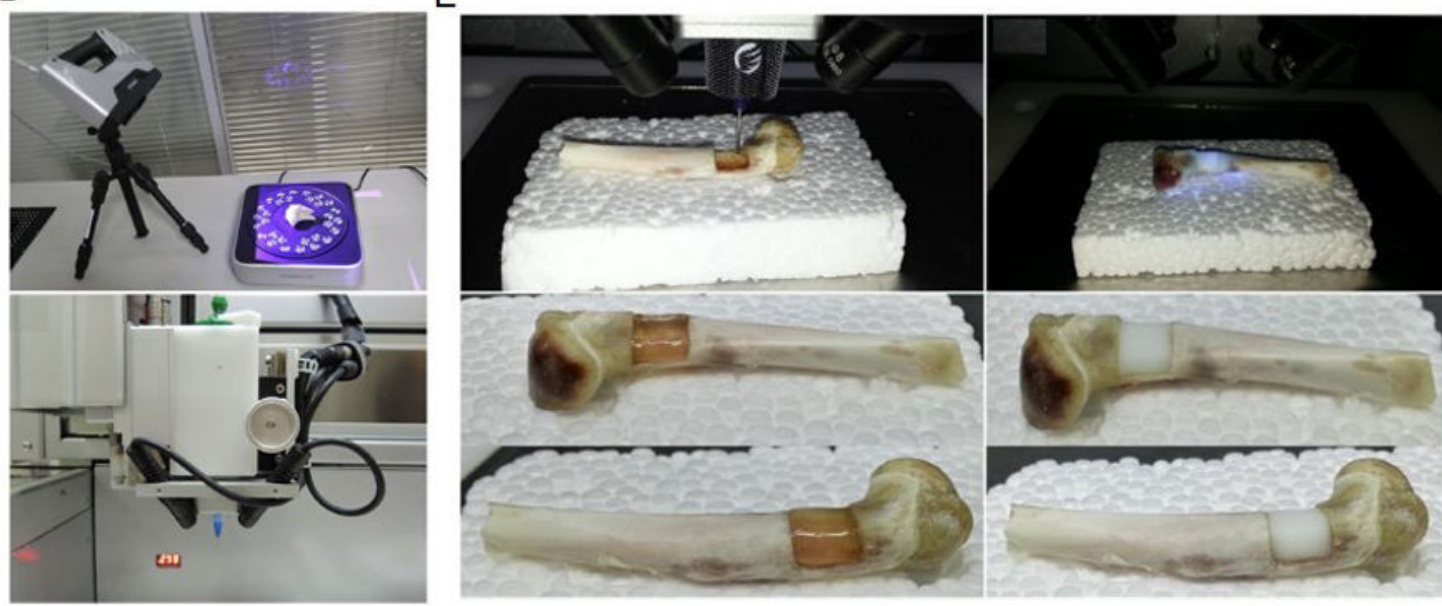

Figure 12.

In situ bioprinting strategies. (A) Schematic describing the in situ bioprinting process of depositing AFSCs directly onto a full-thickness skin wound using laser-guided inkjet bioprinting. (B) Gross histology images illustrating wound closure based on gel only, as well as MSC-encapsulating and AFSC-encapsulating gels in situ bioprinted on top of the skin wound. Reproduced with permission from ref. ${ }^{[185]}$. (C) Immunostaining of ECM molecules elastin, polysaccharides/GAGs, and collagen types I and III in heparin-conjugated HA hydrogels for in situ-bioprinted skins for control, gel only, and gel encapsulating AFSCs. 
Reproduced with permission from ref. ${ }^{[186]}$. (D) (Top) 3D scanner for scanning large-scale bone/cartilage defects and (bottom) nozzle of the 3D bioprinter modified with four UV lights. (E) Process of 3D bioprinting and photopolymerization showing (left) the bioprinting process displaying transparent alginate bioink and (right) the UV photopolymerization process turning hydrogel into a milky color. Reproduced with permission from ref.[187]. 
A
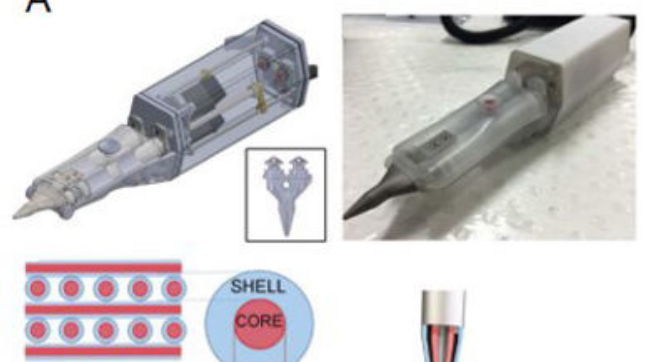

Hydrogel scaffold
Material

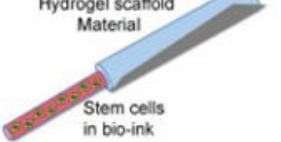

B Left
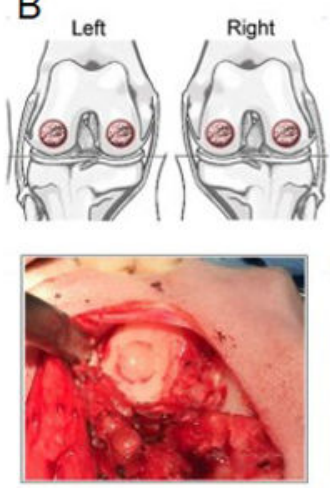

$E$

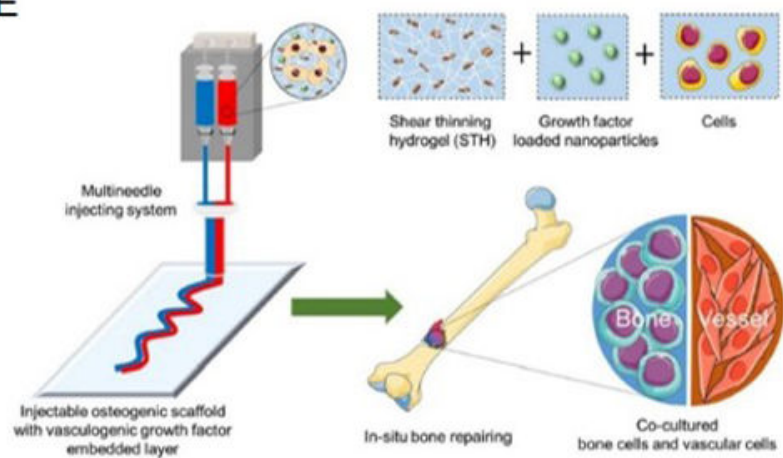

C
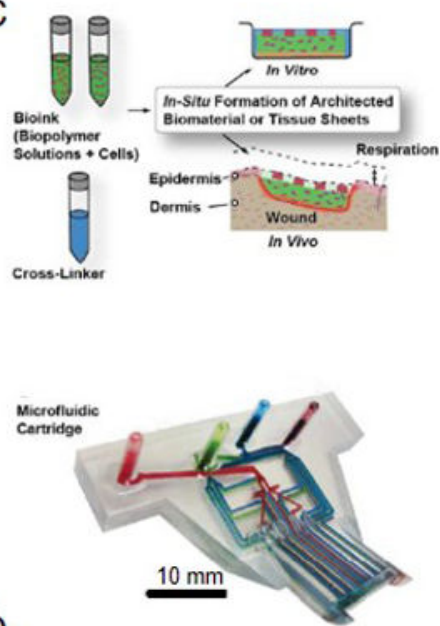

D
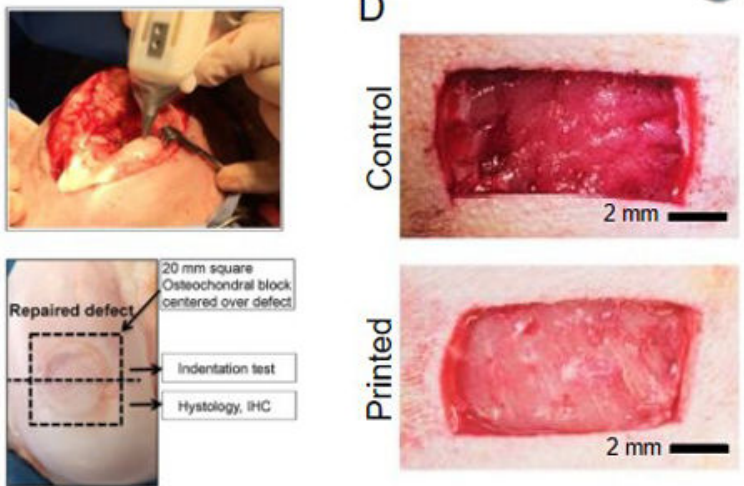
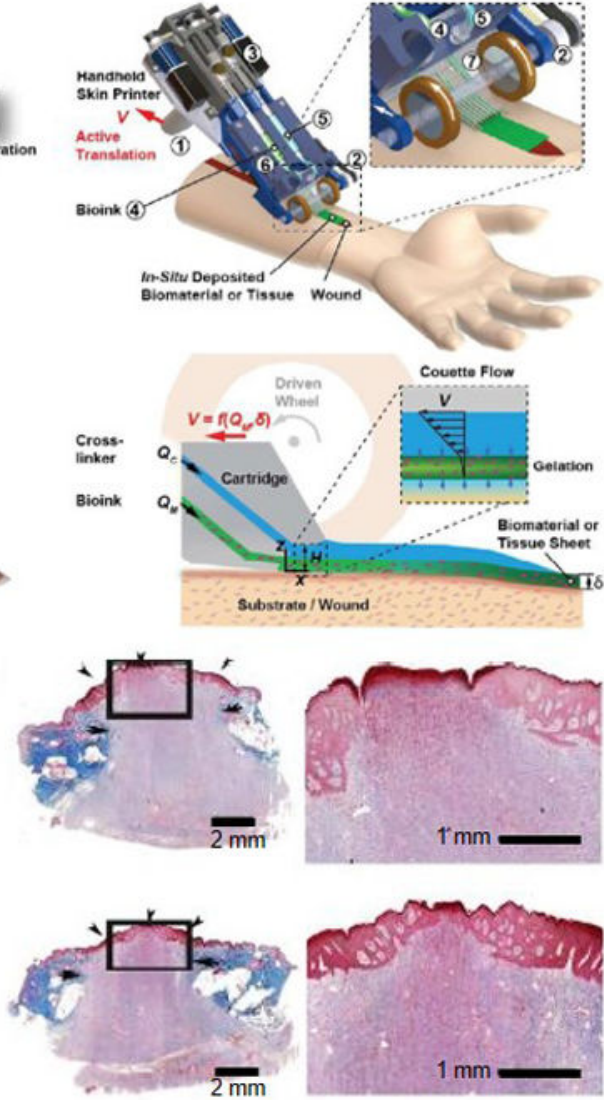

$\mathrm{F}$

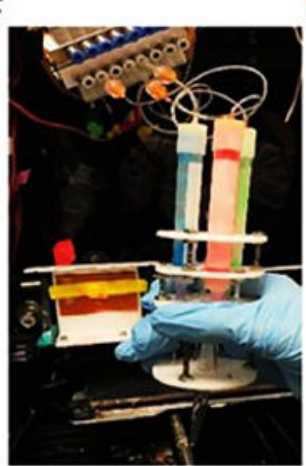

G

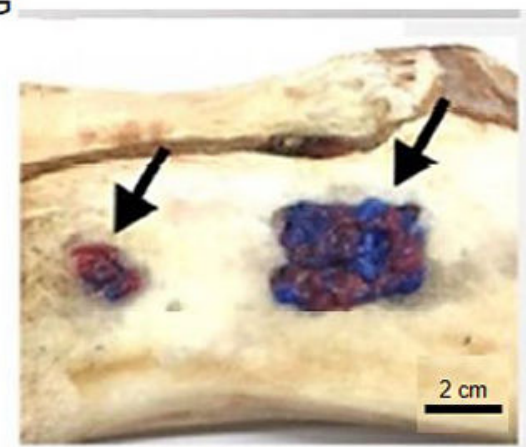

Figure 13.

Handheld in situ bioprinting strategies. (A) (Top) Schematic and photographs of the biopen and (bottom) schematic of the dual extrusion of different cell-laden and support bioinks. (B) Treatment of a full-thickness chondral defect made in the weight bearing area of the medial and lateral femoral condyles using the biopen. Reproduced with permission from ref. ${ }^{[189]}$. (C) Schematic of a handheld skin bioprinter displaying (top left) working principle, (top right) a rendered image of the handheld bioprinter, (bottom left) photograph of the bioink cartridge combining different bioinks, and (bottom right) schematic of the application process of planar skin sheets. (D) Gross images of control and skin sheet-treated group and respective Masson's trichrome staining demonstrating extent of granulation tissue and re- 
epithelization. Reproduced with permission from ref. ${ }^{[190]}$. (E) Schematic illustration of in situ bioprinting using silicate-based shear-thinning hydrogels (STH) encapsulating vasculogenic factor-loaded PCL nanoparticles to treat large-scale bone defects. (F) Photograph of the customized multi-channel handheld bioprinter. (G) Treated large-scale bone defect in a phantom porcine bone using the STH bioink. Reproduced with permission from ref. ${ }^{[191]}$. 
A
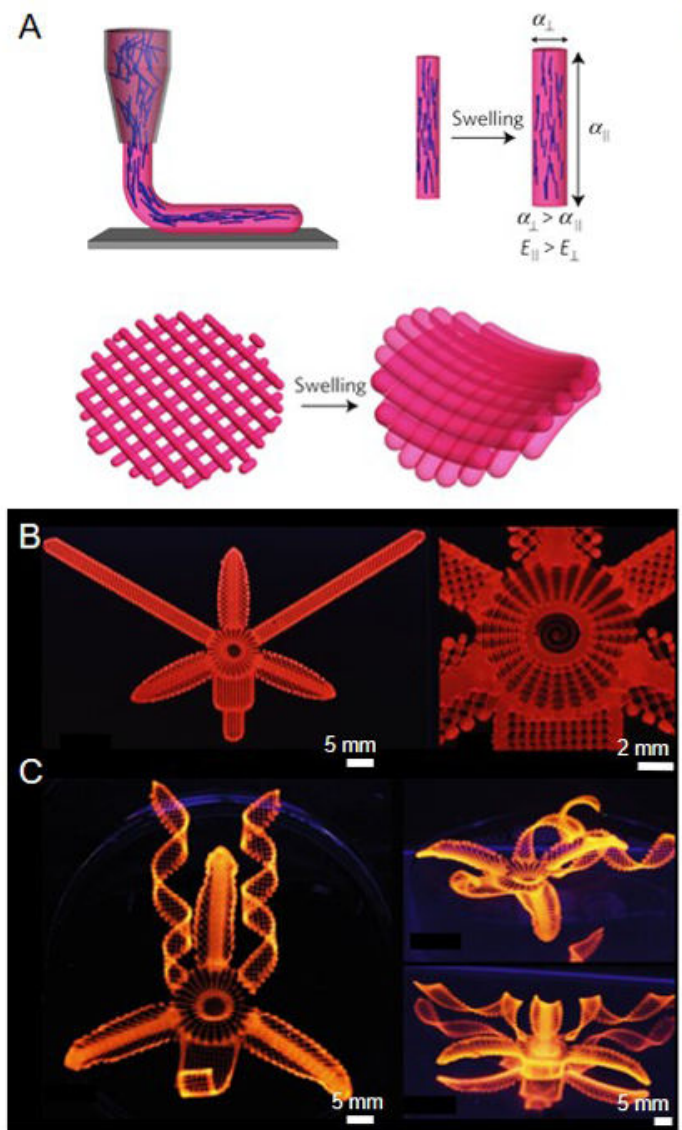

$>E$

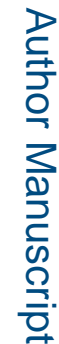

Figure 14.

4D bioprinting. (A) Schematic of a shear-induced alignment of cellulose fibrils during extrusion bioprinting and subsequent effects on anisotropic stiffness and swelling. (B, C) Photographs showing (B) bioprinted and (C) transformed structure of a flower demonstrating the morphology inspired by a native orchid, the Dendrobium helix. Reproduced with permission from ref. ${ }^{[203]}$. (D) Schematics of a magnetically guided printing process and material composition. Ferromagnetic nanoparticles embedded in the composite ink were reoriented by an applied magnetic field generated by a permanent magnet or an electromagnet placed around the dispensing nozzle. (E) A reconfigurable soft electronic device based on an annular ring structure exhibiting different electronic functions depending on the direction of the applied magnetic field. (F) Hexapedal structure stopping and holding a fast-moving object (glass ball with diameter of $18 \mathrm{~mm}$ and weight of $8 \mathrm{~g}$ ) upon application of a magnetic field generated by a permanent magnet. (G) A hexapedal structure wrapping an oblong pharmaceutical pill and carrying the pill using rolling-based locomotion under a rotating magnetic field generated by a permanent magnet. $(\mathrm{H})$ Horizontal leap of a 3D auxetic structure upon sudden reversal of the applied magnetic field direction while attenuating the field strength by rotating a permanent magnet by $90^{\circ}$. Reproduced with permission from ref. ${ }^{[204]}$. 
A

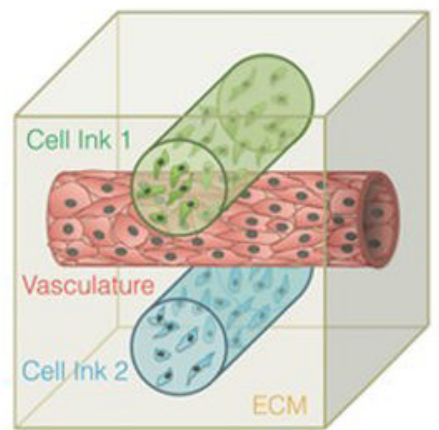

C
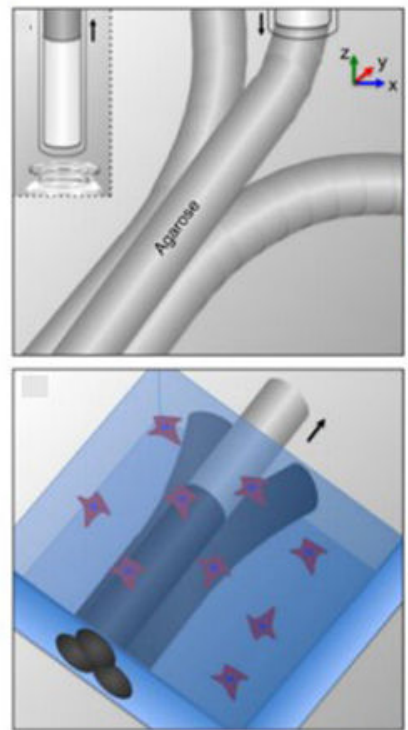

B
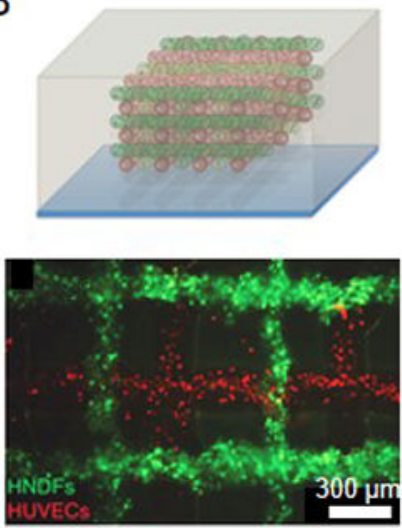

D
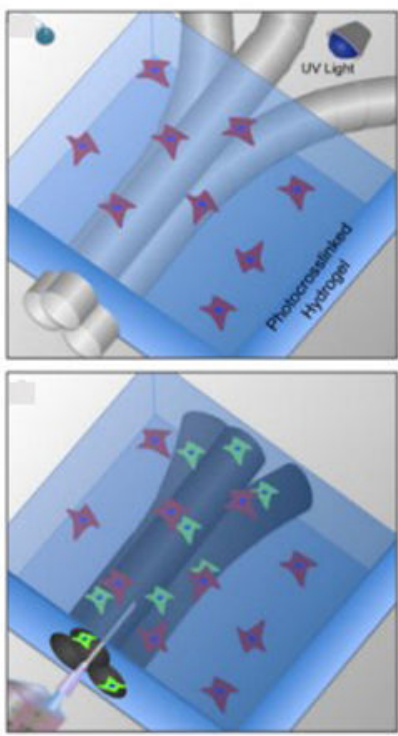
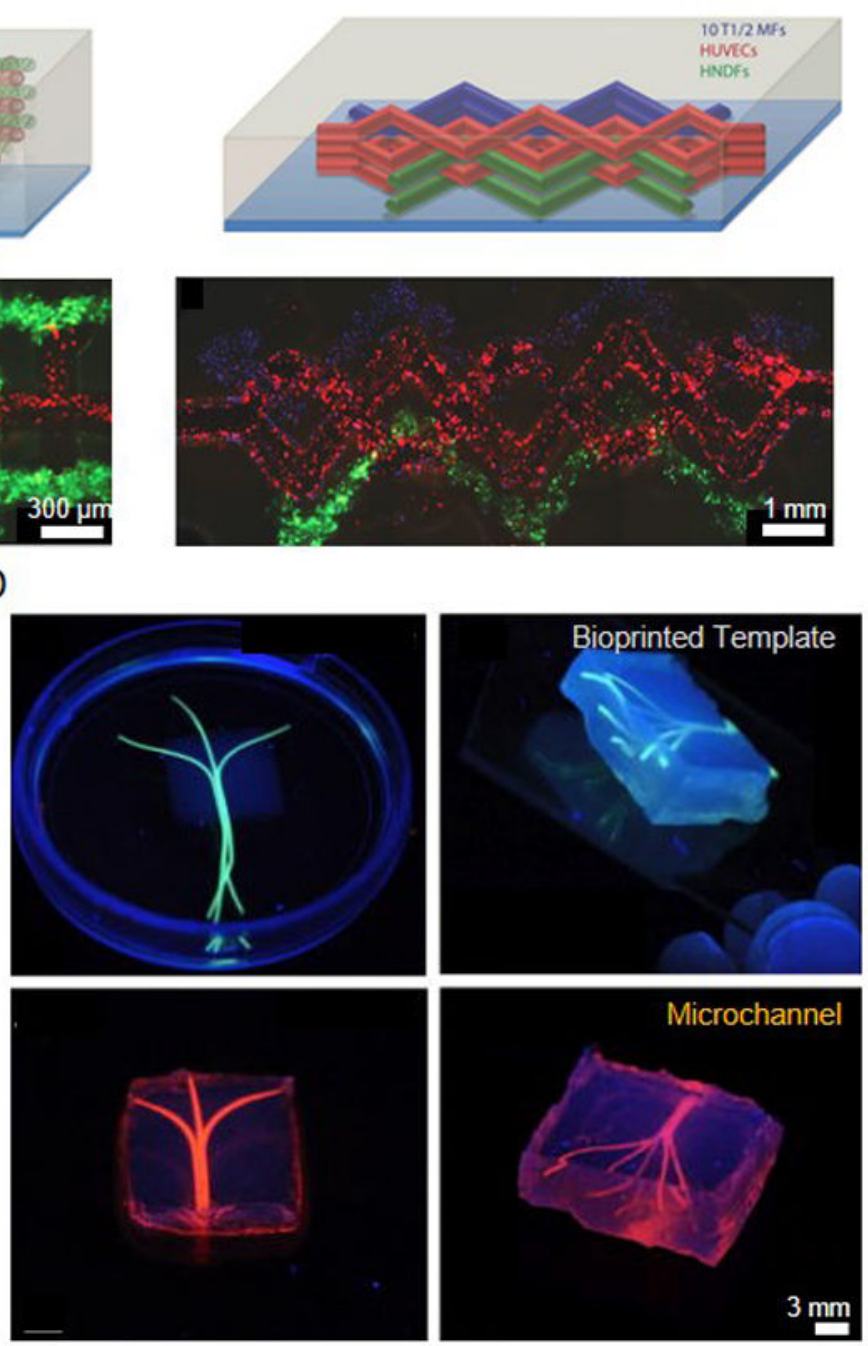

Microchannel

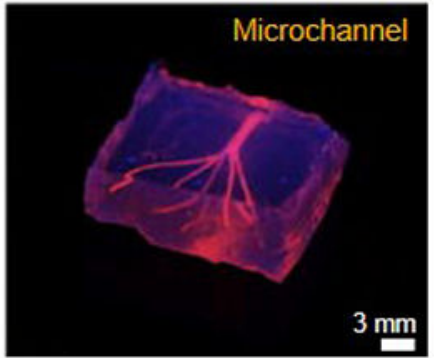

Figure 15.

Extrusion-based sacrificial bioprinting of vascular structures. (A) Schematic views of the approach in which vasculature, cells, and ECM were co-bioprinted to yield engineered tissue constructs composed of heterogeneous subunits. (B) (Top) Schematic side-views and (bottom) fluorescent images of heterogeneous vascularized tissue constructs by adopting Pluronic F-127 as the sacrificial template along with directly extruded tissue bioinks. Reproduced with permission from ref. ${ }^{[175]}$. (C) Schematic representation showing bioprinting of agarose template microfibers and subsequent formation of microchannels via template micromolding. (D) Photographs of the bioprinted template (green) enclosed in a GelMA hydrogel and the respective microchannels perfused with a suspension of fluorescent microbeads (pink). Reproduced with permission from ref. ${ }^{[209]}$. 
A

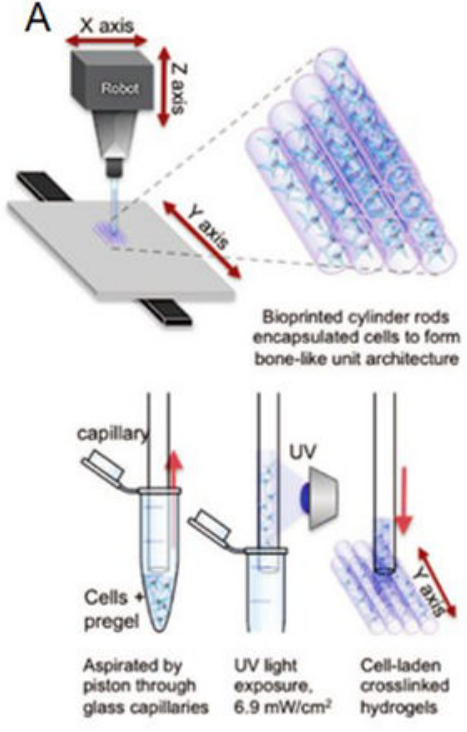

B
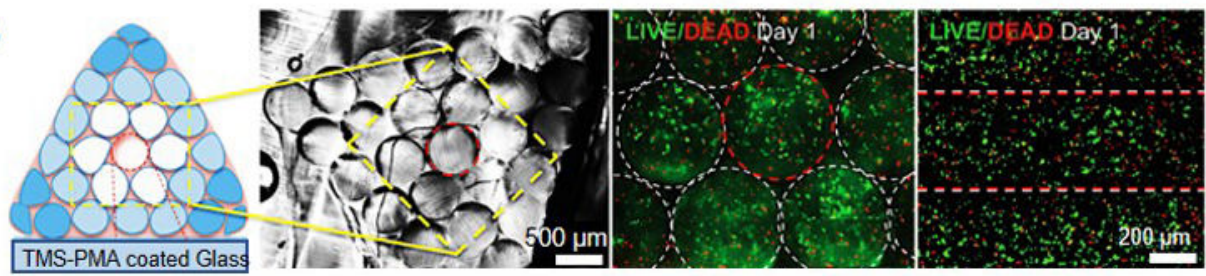

C

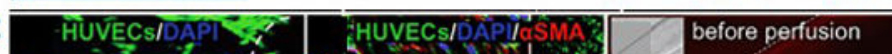

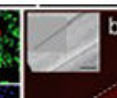

efore perfusion

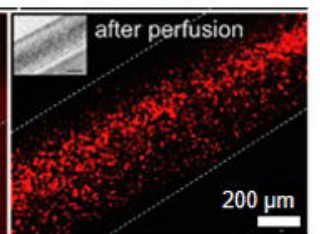

E
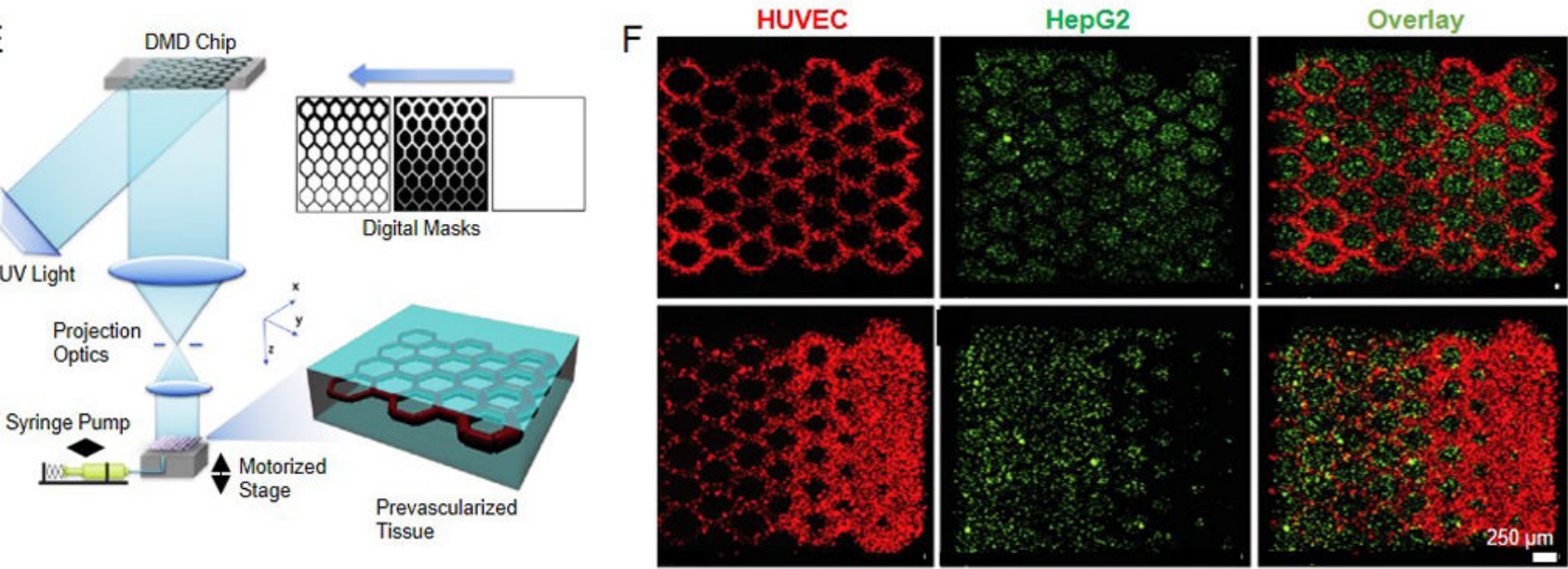

Figure 16.

Direct-write and stereolithography-based bioprinting of vascularized tissues. (A) Scheme of the direct-write bioprinting procedure of independent cell-laden cylinders. (B) The crosssectional view of the entire bioprinted construct. Cross-sectional and top views of the encapsulated cells stained with live/dead inside the construct. (C) Formation and lining of endothelial cells inside the central channel and vascular lumen network perfused with a fluorescent microbeads suspension at day 7 post culture. (D) Production of mineralized ECM in (left two) GelMA/VEGF and (right two) GelMA hydrogels evaluated by Alizarin Red S (ARS) staining. Reproduced with permission from ref. ${ }^{[124]}$. (E) Schematic of a DMDbased bioprinting platform. (F) Fluorescent images demonstrating the bioprinting of heterogeneous cell-laden tissue constructs of HUVECs (red) and HepG2 (green) with (top) uniform and (bottom) gradient vascular channel widths. Reproduced with permission from ref. ${ }^{[213]}$. 


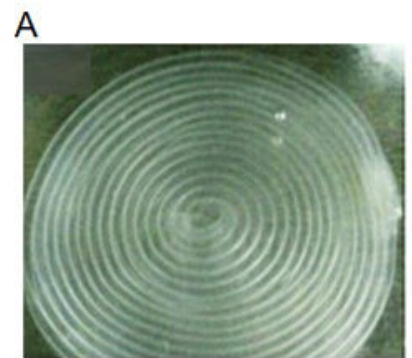

B

C
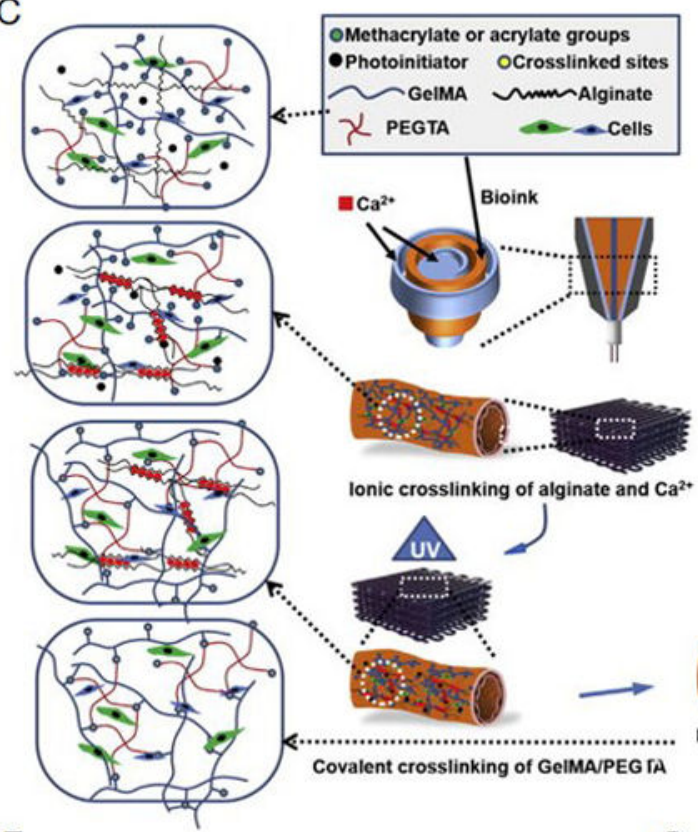

$\mathrm{F}$

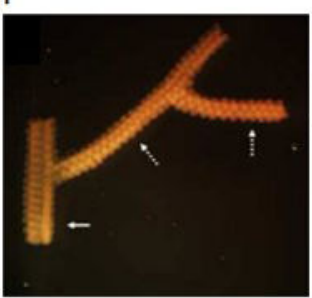

G

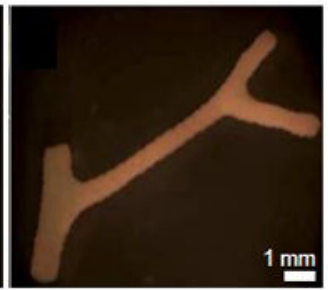

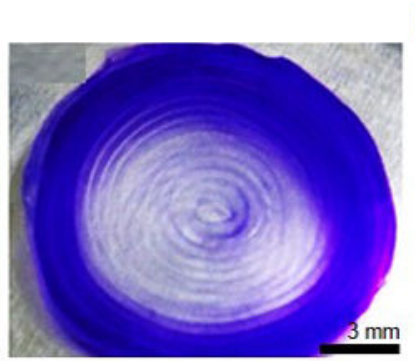
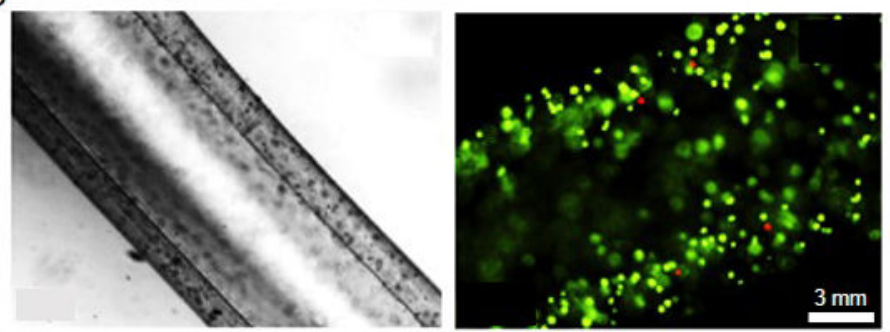

D

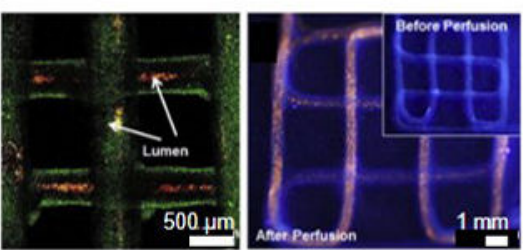

E

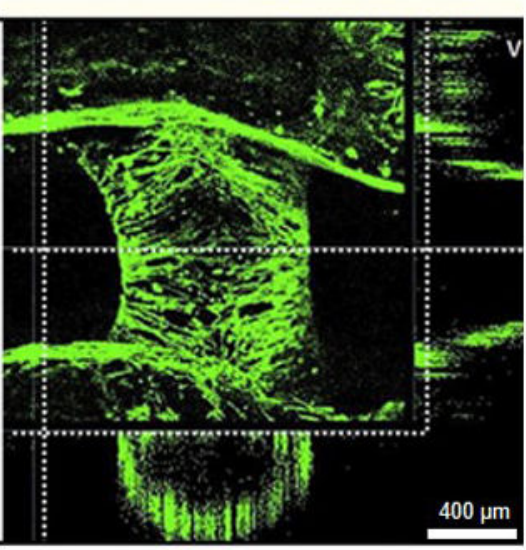

$\mathrm{H}$

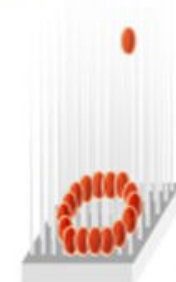

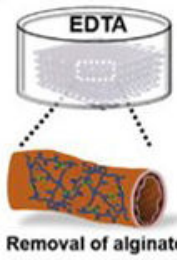
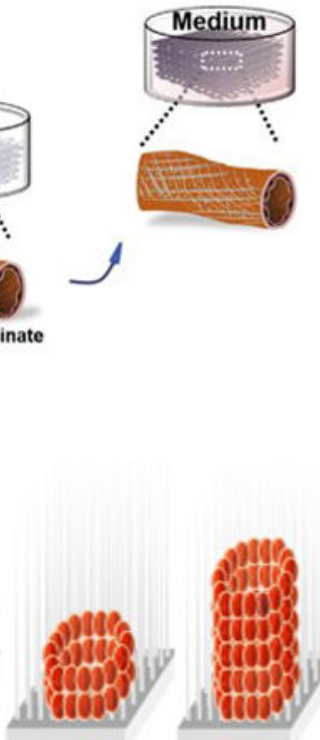
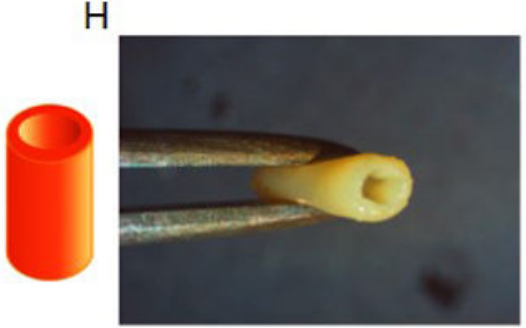

Figure 17.

Bioprinting stand-alone vascular structures. (A) Bioprinted alginate hollow microfibers perfused with a purple dye. (B) (Left) microscopic images showing the hollow channel and L929 mouse fibroblast-laden wall of the bioprinted hollow alginate microfiber and (right) live (green)/dead (red) staining of the cells. Reproduced with permission from ref. ${ }^{[214]}$. (C) Schematic diagram showing two independent crosslinking processes of a composite bioink, where alginate and GelMA/PEGTA were ionically and covalently crosslinked, respectively, upon exposure to $\mathrm{CaCl}_{2}$ solution and $\mathrm{UV}$ light, as well as representations of bioprinting processes including (ci) differently designed nozzles and (cii) different layers. (D) Fluorescence micrograph and photographs showing injection of red fluorescent microbeads into the lumen of a single, continuous bioprinted tube. (E) Representative confocal micrograph showing f-actin staining of encapsulated vascular cells after 21 days of culture 
post-bioprinting in the 3D tubular construct. Reproduced with permission from ref. ${ }^{[216]}$. (F) Multi-cellular spheroids assembled into a tubular structure, which underwent fusion to form an integral blood vessel. Reproduced with permission from ref. ${ }^{[217]}$. (G) Schematic of the "Kenzan" method in which cell aggregates were sewed onto an array of needles based on a computer program. (H) Cell aggregates eventually fused to form a uniform layer in the wall of a blood vessel. Reproduced with permission from ref. ${ }^{[219]}$. 
A

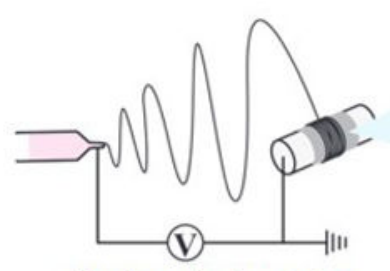

Electrospinning Setup

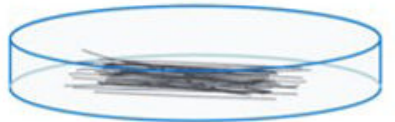
Solution

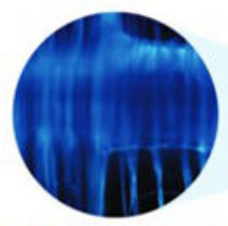

Formation of 3D neural scaffold embedded with electrospun fibers

D
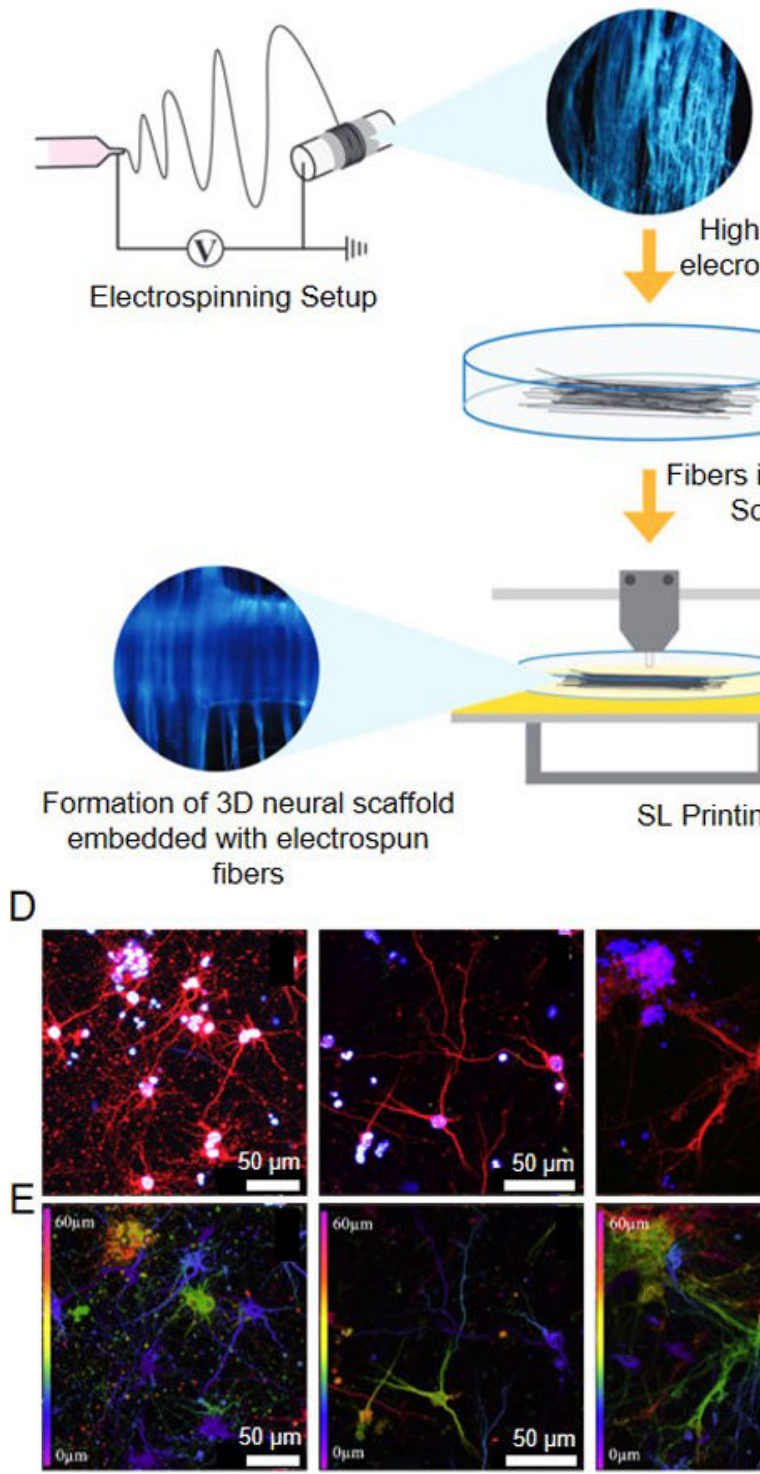

Highly aligned elecrospun fibers

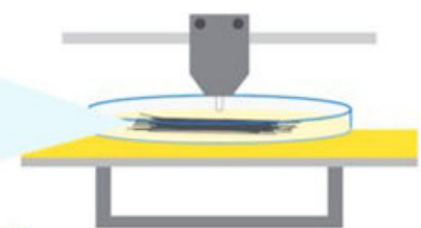

SL Printing Scaffold

F
Fibers in PEG-DA

\section{s}
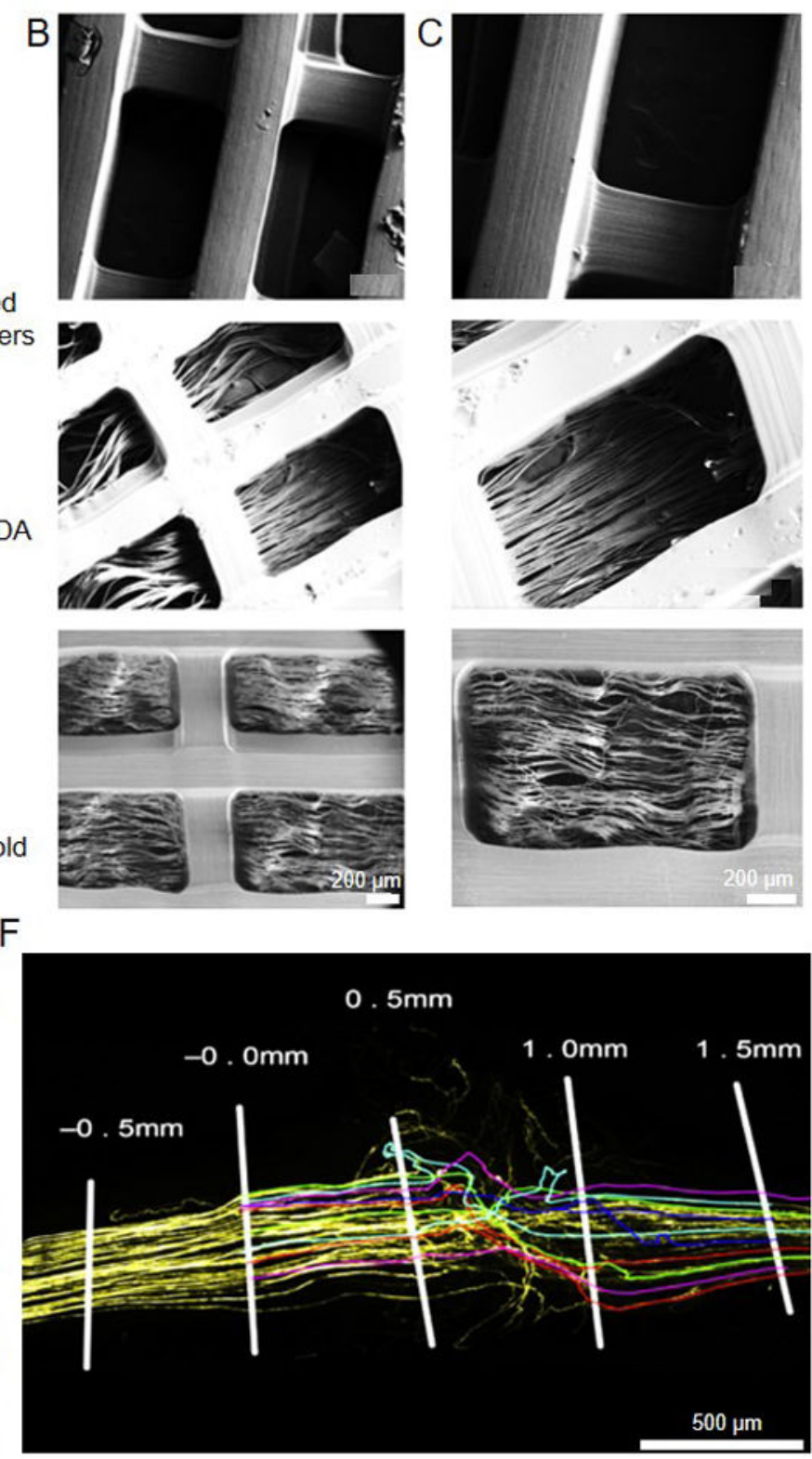

Figure 18.

Bioprinting of neural structures and nerve guides. (A) Schematic diagram of the 3D bioprinting process of a neural scaffold. (B, C) SEM images of (top) PEG-DA scaffolds with large porosity of $66 \%$ fabricated by stereolithography-based bioprinting, (middle) electrospun PCL fibers attached to the bioprinted PEG-DA scaffold, and (bottom) electrospun PCL/gelatin fibers attached to the bioprinted PEG-DA scaffold. Reproduced with permission from ref. ${ }^{[223]}$. (D, E) 3D-bioprinted brain like structures made of gellangum-RGD: (D cortical neurons incorporated into hydrogels of different concentration stained with BIII tubulin (red) for cortical neurons and DAPI (blue) for nuclei; (E) confocal images of neurons, where the different colors represent the depth of the neurons from $0 \mu \mathrm{m}$ (blue) to $60 \mu \mathrm{m}$ (red). Reproduced with permission from ref. ${ }^{[221]}$. (F) Fluorescence micrograph showing the repair of a peripheral nerve supported by a 3D-bioprinted nerve 
guide made of PEG after implantation into mice with an induced small gap nerve injury. Different pseudo-colors represent individually tracked axons that displayed re-connection at 21 days post-implantation. Reproduced with permission from ref. ${ }^{[222]}$. 

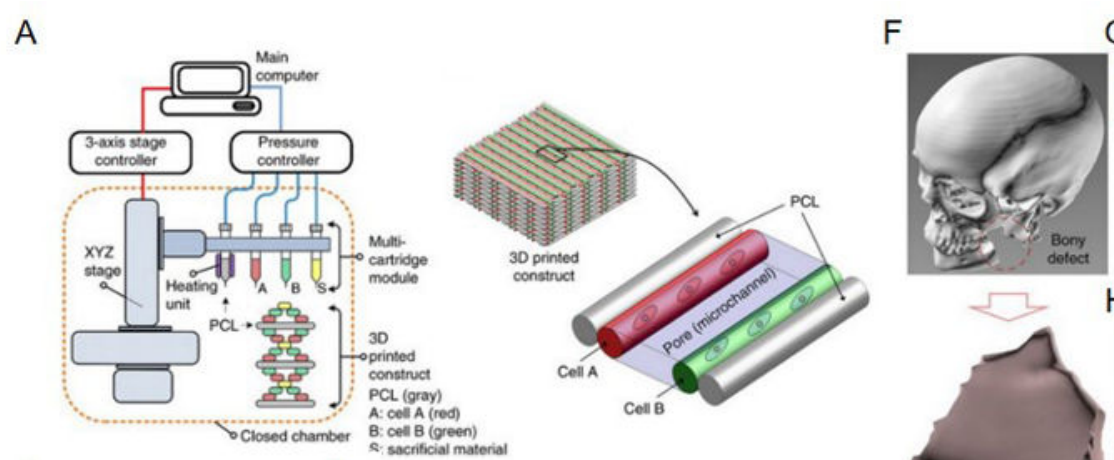

G

B

C
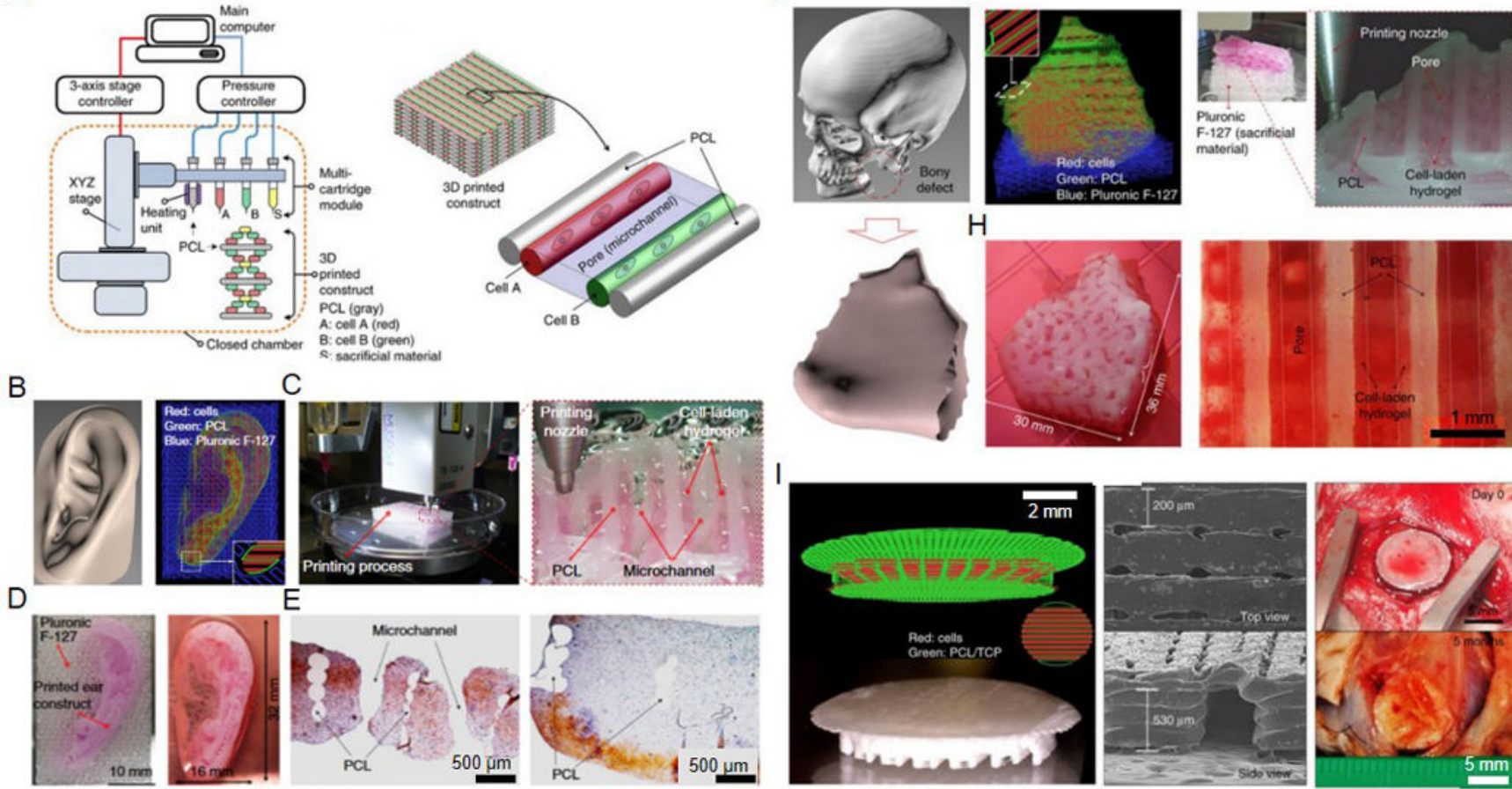

Figure 19.

Bioprinting of human-scale cartilage and bone constructs. (A) Schematic of a bioprinting system termed "ITOP" and illustration of basic patterning of a 3D architecture including multiple cell-laden hydrogels and PCL support polymer. (B) (Left) 3D CAD model and (right) 3D visualized motion program of a human ear. (C) The 3D bioprinting process of a human ear construct using the ITOP method. (D) Photographs of the bioprinted cartilage ear (left) before and (right) after removal of sacrificial Pluronic F127. (E) Safranin-O staining of the bioprinted cartilage ear construct (left) with microchannels and (right) without microchannels after culture in chondrogenic medium for 5 weeks. (F) The 3D CAD model of a mandible bone defect from human CT image data. (G) (Left) 3D visualized motion program displaying the paths of different bioinks in different colors and (right) the 3D bioprinting process. $(\mathrm{H})$ (Left) photograph of bioprinted mandible bone defect construct and (right) osteogenic differentiation of AFSCs in the bioprinted constructed as visualized by ARS staining. (I) (Left) visual motion program and photograph a bioprinted calvarial bone defect, (middle) SEM images of the bioprinted bone construct at (top) day 0 and (bottom) 5 months post-implantation into a rat model, and (right) photographs of the implanted calvarial bone defect construct at (top) day 0 and (bottom) 5 months post-implantation into a rat model. Reproduced with permission from ref. ${ }^{[64]}$. 
A

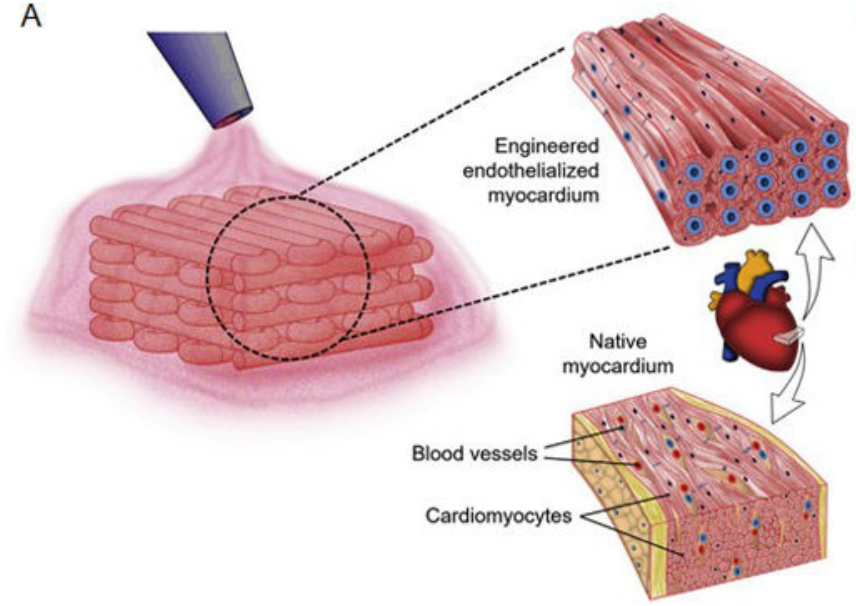

E

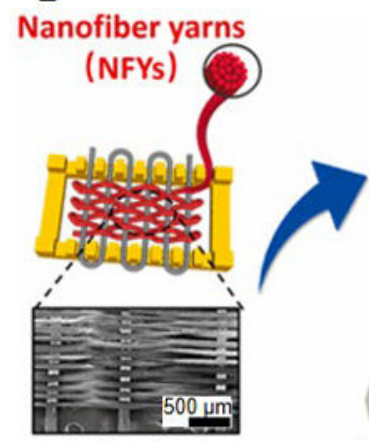

NFYs-NET
B

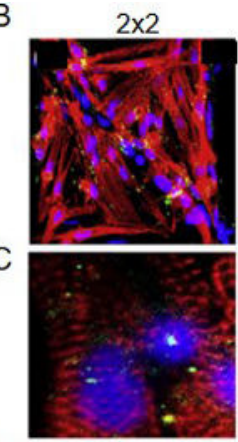

D

Hydrogel precursor

solution

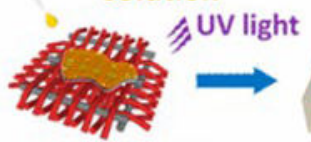

NFYs-NET/Hydrogel 3D scaffold

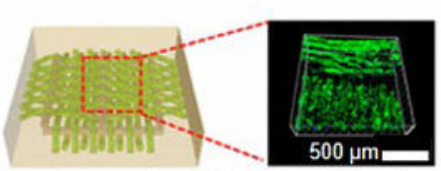

Engineered 3D Cardiac Anisotropy

F

G
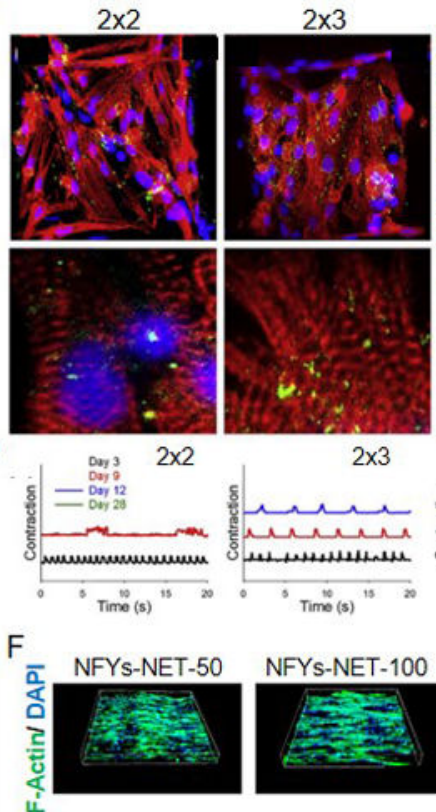

$2 \times 3$
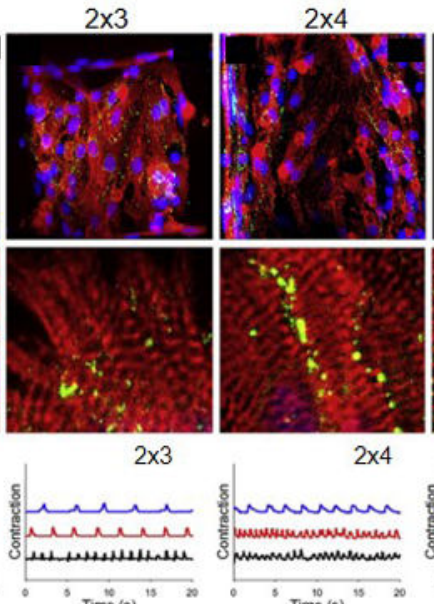

$2 \times 4$

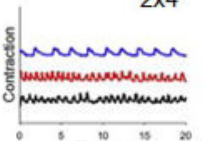

n

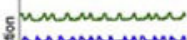

rinniman

nominumanom

- $\operatorname{Mime}^{10}$ (s) 15
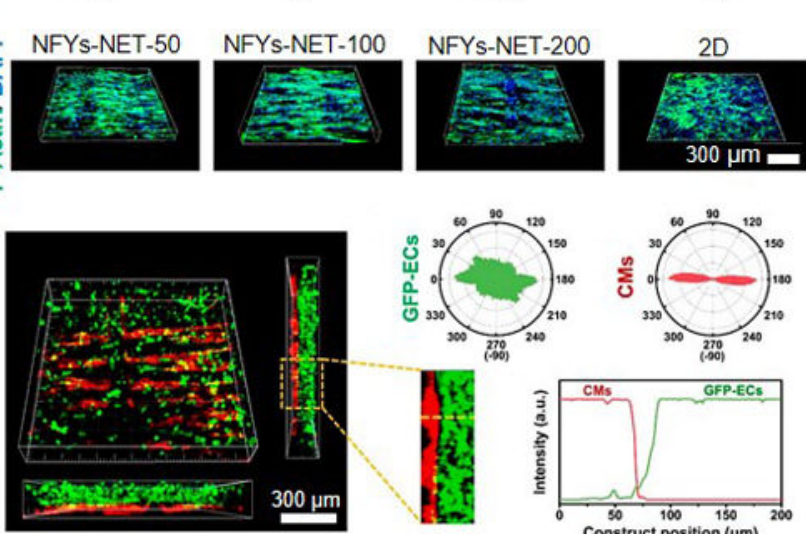
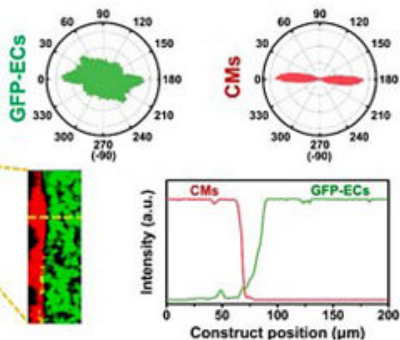

Figure 20.

Bioprinting of cardiac tissues. (A) Schematic representation of seeding cardiomyocytes onto a scaffold containing endothelial cells to obtain an endothelialized myocardium. (B) Immunofluorescence staining of sarcomeric a-actinin (red) and Cx43 (green) of cardiomyocytes seeded on bioprinted microfibrous scaffolds with different aspect ratios of unit grids. (C) Magnified images showing the sarcomeric banding. (D) Beating analysis of the cardiac organoid on bioprinted scaffolds with different aspect ratios of unit grid. Reproduced with permission from ref. ${ }^{[234]}$. (E) Schematic representation of a bioprinting process based on nanofiber yearns to obtain 3D engineered cardiac anisotropy. (F) 3D views of fluorescent images of cardiomyocytes on NFYs-NET scaffolds by staining for f-actin (green) and nuclei (blue) after 5 days of cultivation. (G) 3D view in confocal images of GFP-endothelial cells (ECs, green) and cardiomyocytes (CMs, red) within scaffolds and the quantitative analysis of cellular orientation distribution of GFP-ECs and CMs, as well as the fluorescence intensity analysis for the cross-section of this cell-laden scaffold showing the distribution of ECs in hydrogel and CMs on NFYs-NET. Reproduced with permission from ref. ${ }^{[235]}$. 
A

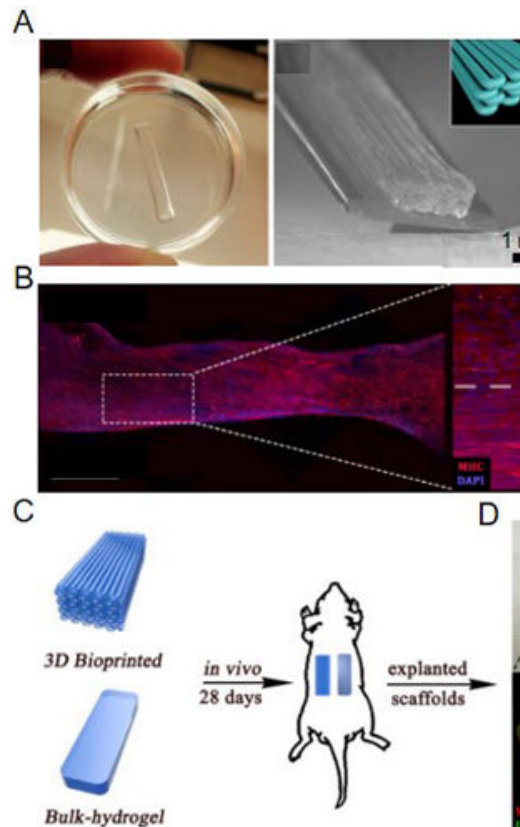

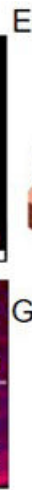
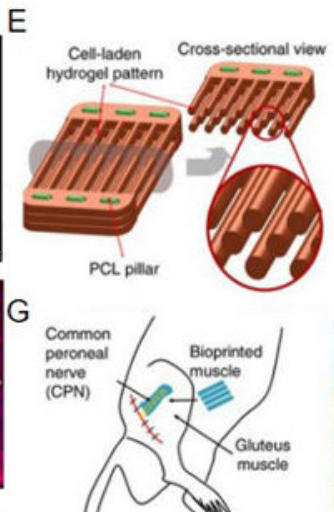

PCL pillar
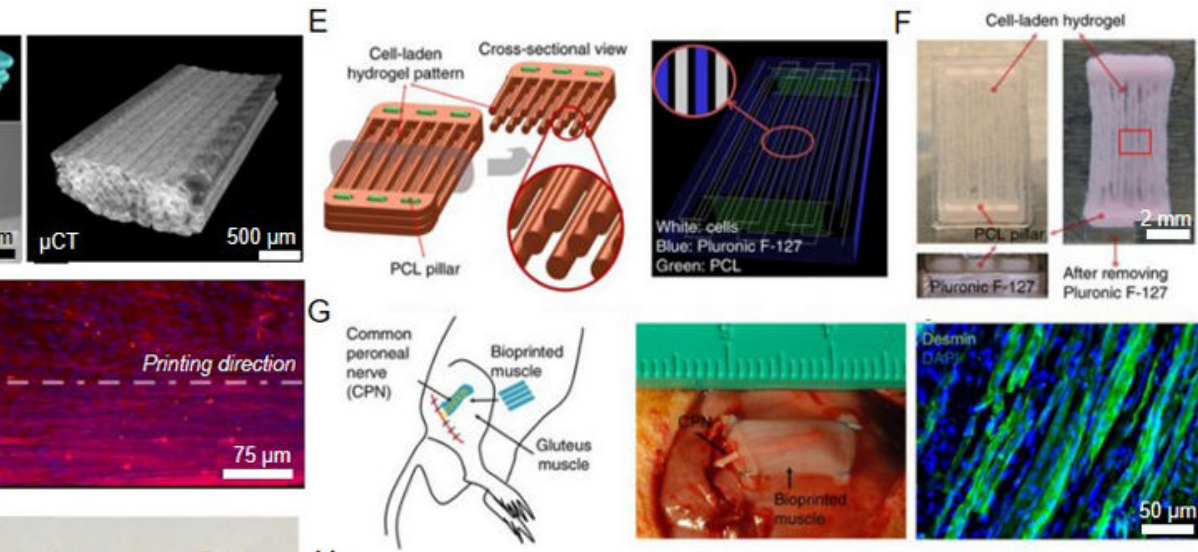

$\mathrm{H}$
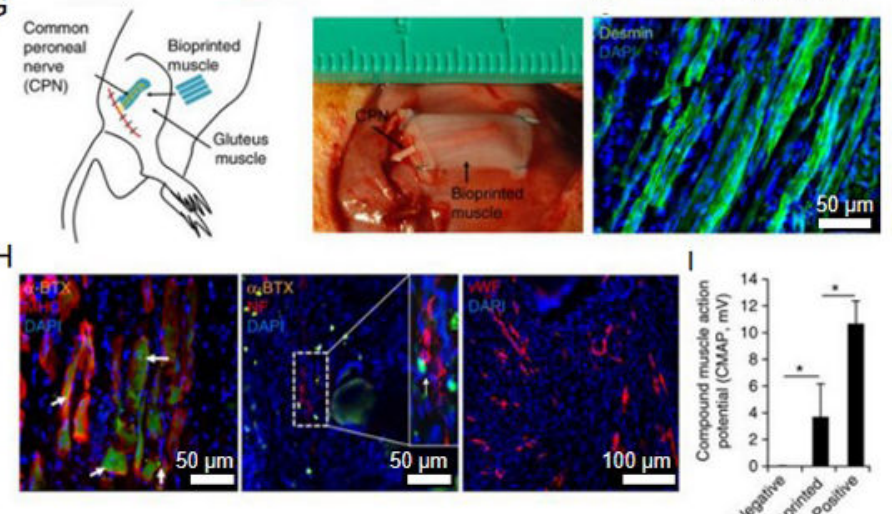

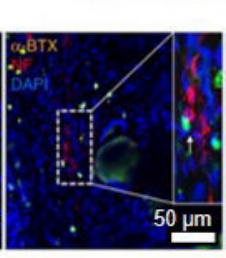

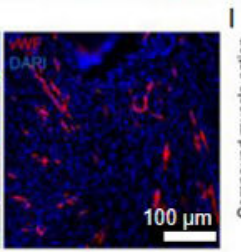

$100 \mu \mathrm{m}$

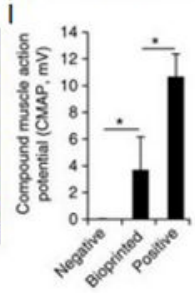

Figure 21.

Bioprinting of skeletal muscle tissues. (A) (Left) photograph, (middle) micrograph, and (right) microCT image of a 3D-bioprinted PEG-fibrinogen scaffold for skeletal muscle fabrication. (B) Immunofluorescent staining of muscle networks displaying alignment of formed myotubes after 15 days of in vitro culture. (C) Schematic of the implantation of 3D muscle constructs into the back of immunocompromised SCID mice. (D) (Top) photographs and (bottom) immunofluorescent staining images against MHC (red) and LAM (green) of the explants after 28 days of in vivo culture. Reproduced with permission from ref. ${ }^{[238]}$. (E) (Left) schematic showing the designed fiber bundle structure for bioprinted skeletal muscle organization and (right) 3D visualized motion program of the different bioinks within the construct. (F) The 3D patterning outcome of designed muscle organization (left) before and (after) removing sacrificial Pluronic F127 using the ITOP bioprinter. (G) (Left) schematic diagram and (middle) photograph of ectopic implantation of bioprinted muscle construct in vivo. (Right) immunostaining of skeletal muscle marker desmin to confirming the presence of organized muscle fibers and innervating capability. $(\mathrm{H})$ Immunostaining of (left) $\mathrm{MHC}^{+}$ (red) and $a$-BTX ${ }^{+}$(orange) structures, (middle) neurofilaments (NF, red) and a-BTX ${ }^{+}$ (orange) structures, and (right) van Willebrand factor (red). Nuclei were stained with DAPI (blue) in all images. (I) Functional assessment of the bioprinted muscle constructs after 4 weeks of implantation. Reproduced with permission from ref. ${ }^{[64]}$. 


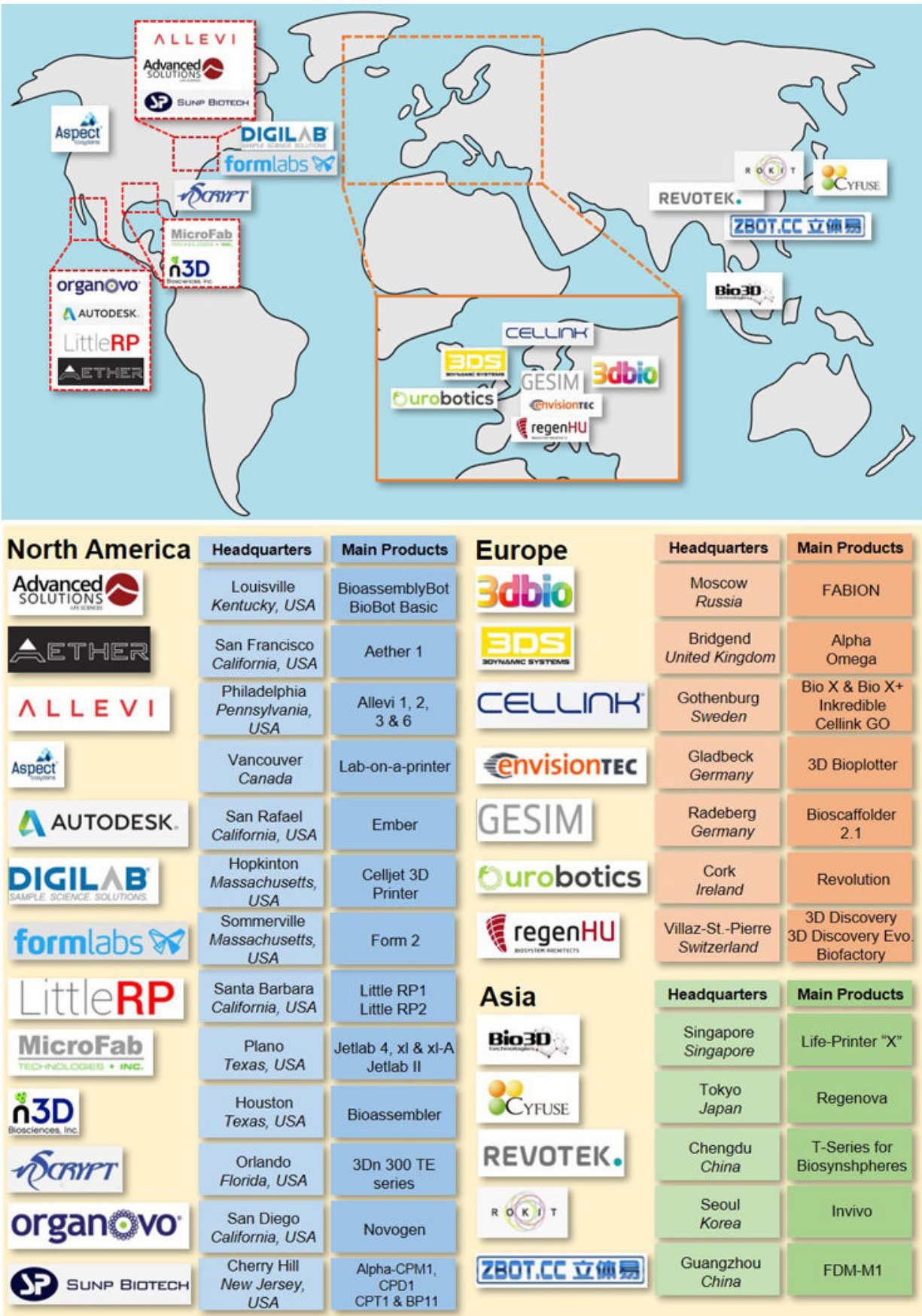

Figure 22.

Overview of bioprinting companies illustrating the locations headquarters and main products. 
Table 1:

Overview of advantages and disadvantages of different bioprinting techniques.

\begin{tabular}{|c|c|c|}
\hline $\begin{array}{l}\text { 3D Bioprinting } \\
\text { Technique }\end{array}$ & Advantages & Disadvantages \\
\hline Stereolithography & $\begin{array}{l}\text { - Simultaneous crosslinking of the whole } 2 \mathrm{D} \text { layer avoids } \\
\text { need of X-Y movement } \\
\text { - High cell viability }(>85 \%) \\
\text { - High variety of printable bioinks } \\
\text { - High resolution of bioprinting }(\sim 1 \mu \mathrm{m})\end{array}$ & $\begin{array}{l}\text { - Crosslinking requires transparent and } \\
\text { photosensitive bioink limiting choice of additives and } \\
\text { cell density }\left(10^{8} \text { cells } \mathrm{mL}^{-1}\right) \\
\text { - Comparatively complex system }\end{array}$ \\
\hline Inkjet Bioprinting & $\begin{array}{l}\text { - Simple bioprinting method } \\
\text { - Low cost } \\
\text { - Applicability of multi-material bioprinting } \\
\text { - High resolution }(\sim 30 \mu \mathrm{m}) \\
\text { - High cell viability }(80-90 \%)\end{array}$ & $\begin{array}{l}\text { - Limited to low cell density }\left(<10^{6} \text { cells } \mathrm{mL}^{-1}\right) \\
\text { - Limited to bioinks with viscosity of } 3.5-12 \mathrm{mPa} \cdot \mathrm{s}\end{array}$ \\
\hline $\begin{array}{l}\text { Laser-assisted } \\
\text { Bioprinting }\end{array}$ & $\begin{array}{l}\text { - High cell viability }(>95 \%) \\
\text { - Variety of printable bioinks with viscosity of } 1-300 \\
\mathrm{mPa} \cdot \mathrm{s}\end{array}$ & $\begin{array}{l}\text { - Limited to low cell density }\left(<10^{6} \text { cells } \mathrm{mL}^{-1}\right) \\
\text { - Complex system } \\
\text { - Comparatively high costs }\end{array}$ \\
\hline $\begin{array}{l}\text { Extrusion-based } \\
\text { Bioprinting }\end{array}$ & $\begin{array}{l}\text { - Printability of highly viscous bioinks }\left(30-6 \times 10^{7} \mathrm{mPa} \cdot \mathrm{s}\right) \\
\text { - Printability of high cell densities (including cell } \\
\text { spheroids) } \\
\text { - Applicability of multi-material bioprinting } \\
\text { - Comparatively simple bioprinting process }\end{array}$ & $\begin{array}{l}\text { - Relatively low printing speed } \\
\text { - Low-to-medium resolution highly dependent on } \\
\text { setup } \\
\text { - Moderate cell viability (40-80\%) dependent on } \\
\text { setup }\end{array}$ \\
\hline $\begin{array}{l}\text { Electrospinning-based } \\
\text { Bioprinting }\end{array}$ & $\begin{array}{l}\text { - High resolution }(<1 \mu \mathrm{m}) \\
\text { - Optimal for the fabrication of scaffolds }\end{array}$ & $\begin{array}{l}\text { - Not possible to directly bioprint cell-laden } \\
\text { constructs } \\
\text { - Complex system } \\
\text { - High costs }\end{array}$ \\
\hline
\end{tabular}

